\title{
Application of Neural Networks to Waste Site Screening
}

\author{
A. E. Dabiri \\ M. Garrett \\ T. Kraft \\ J. Hilton
}

M. VanHammersveld

Published February 1993
Idaho National Engineering Laboratory
EG\&G Idaho, Inc.
Idaho Falis, Idaho 83415

Prepared for the

U.S. Department of Energy

Office of Environmental Restoration and Waste Management

Under DOE Idaho Field Office

Contract DE-AC07-76ID01570

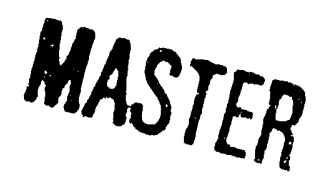




\section{ABSTRACT}

Waste site screening requires knowledge of the actual concentrations of hazardous materials and rates of flow around and below the site with time. The present approach to site screening consists primarily of drilling boreholes near contaminated sites and chemically analyzing the extracted physical samples and processing the data.

The present approach to site screening is expensive and time consuming. The feasibility of using neural network techniques to reduce the cost of waste site screening was investigated. Two neural network iechniques, gradient descent back propagation and fully recurrent back propagation were utilized. The networks were trained with data received from Westinghouse Hanford Corporation. The results indicate that the network trained with the fully recurrent technique shows satisfactory generalization capability. The predicted results are close to the results obtained from a mathematical flow prediction model. It is possible to develop a new tool to predict the waste plume, thus substantially reducing the number of the bore sites and samplings.

There are a variety of applications for this technique in environmental site screening and remediation. One of the obvious applications would be for optimum well siting. A neural network trained from the existing sampling data could be utilized to decide where would be the best position for the next bore site. Other applications are discussed in the report. 


\section{SUMMARY}

The work described in this report investigated the feasibility of using neural network techniques to reduce the cost of waste site screening. The successful technique would reduce the number of boreholes and the sampies analyzed from each borehole to properly screen the waste site. The analytic tool development described is inexpensive because it makes use of neural network techniques that can interpolate rapidly and learn how to analyze data rather than having to be explicitly programmed.

A neural network is an interconnected collection of units that behave like neurons in the brain. Each unit receives multiple normalized input signals and generates a single output signal. The output from each unit is typically connected as other input to other units in the network. The connections are weighted so that the output of a unit is the weighted sum of the activation signals passed through a nonlinear threshold function. A subset of the units receives input from external sources, and another subset of units generates output for the network, so that the entire neural network can be viewed as a nonlinear mapping between a vector of input activations to a vector or output values.

The connection weights among the units represent the nonlinear mapping function for the neural network. The weights are determined through training where the weights are incrementally adjusted over multiple training cycles. Once the neural network is trained, it is capable of recalling the mapping by feeding the input activation forward through the network to the output. The types of units, connection topology among the units, propagation of signals through the network and learning algorithm are defined by the selected neural network paradigm: back propagation and fully recurrent neural networks. Back propagation is an effective mapping function if the relationship between the input activation vector and output activation vector is a continuous nonlinear mapping. The fully recurrent network effectively maps multiple time series sequences at the input to one or more output sequences at the output.

The neural network approach has two advantages over analytical models. First, the neural network attempts to capture the large scale behavior of the process and progresses to the fine scale behavior if the information is available. Analytical models are typically constructed from the bottom up extending the micro model in steps to a macro model description of the site. The second advantage is that once a neural network has successfully been applied at one site, the same process can be used to screen other sites. The neural network approach is adaptable and can be tuned to a specific site.

Applying fully recurrent neural network to site data is promising for a general purpose site screening tool. To date, recurrent neural networks have been developed that are able to capture the dynamics at different depths and radii from the injection site, generalize to different radials, account for local variations in soil conditions, and are independent of injection time and rate, In its current configuration, the recurrent network reduces the number of test bore hole sites by extending the accuracy of predictions over more widely spaced test sites. 


\section{CONTENTS}

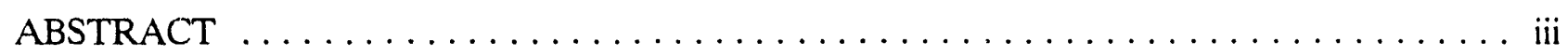

SUMMARY $\ldots \ldots \ldots \ldots \ldots \ldots \ldots \ldots \ldots \ldots \ldots \ldots \ldots \ldots \ldots \ldots \ldots \ldots$

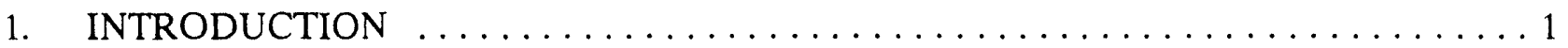

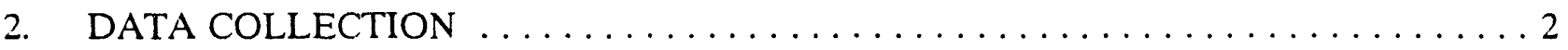

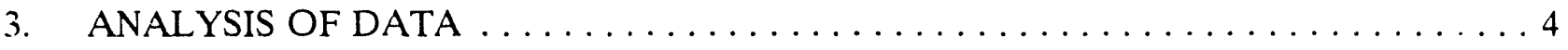

4. NEURAL NETWORKS TECHNIQUES $\ldots \ldots \ldots \ldots \ldots \ldots \ldots \ldots \ldots$

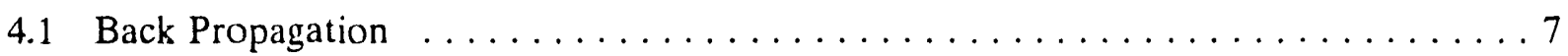

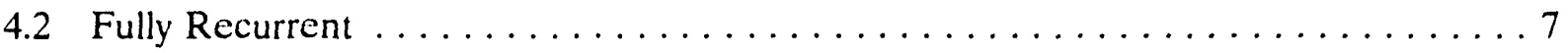

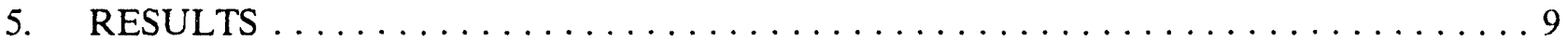

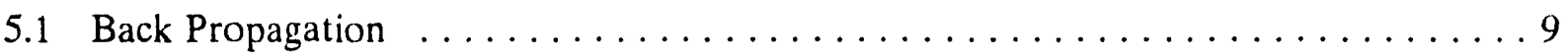

5.2 Fully Recurrent Network $\ldots \ldots \ldots \ldots \ldots \ldots \ldots \ldots \ldots \ldots \ldots \ldots \ldots \ldots \ldots$

6. DISCUSSION OF RESULTS $\ldots \ldots \ldots \ldots \ldots \ldots \ldots \ldots \ldots \ldots \ldots \ldots \ldots$

7. APPLICATIONS $\ldots \ldots \ldots \ldots \ldots \ldots \ldots \ldots \ldots \ldots \ldots \ldots \ldots \ldots \ldots$

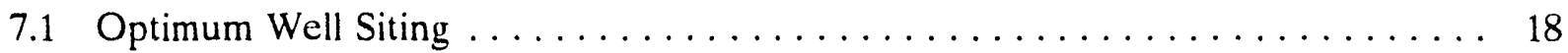

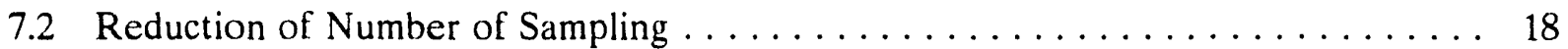

7.3 Reduction of Number of Bore Sites $\ldots \ldots \ldots \ldots \ldots \ldots \ldots \ldots \ldots \ldots$

8. CONCLUSIONS AND RECOMMENDATIONS $\ldots \ldots \ldots \ldots \ldots \ldots \ldots \ldots$

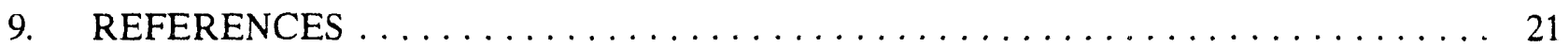

Appendix $\mathrm{A}-$ Computer Source Programs Listing $\ldots \ldots \ldots \ldots \ldots \ldots \ldots \ldots \ldots \ldots \ldots$

Appendix B-Back Propagation Network Process Development $\ldots \ldots \ldots \ldots \ldots \ldots$. . . .

Appendix C-Fully Recurrent Network Process Development $\ldots \ldots \ldots \ldots \ldots \ldots \ldots$. . . . 


\section{FIGURES}

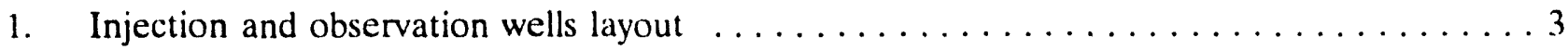

2. Neural network algorithm application process $\ldots \ldots \ldots \ldots \ldots \ldots \ldots \ldots \ldots \ldots$

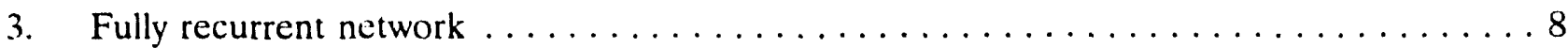

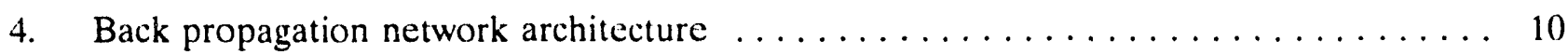

5. Fully recurrent network architecture (three to six matrix) $\ldots \ldots \ldots \ldots \ldots \ldots$

6. Actual versus predicted moisture values, radial $\mathrm{A}$, distance $1 \mathrm{~m} \ldots \ldots \ldots$

7. Actual versus predicted moisture values, radial $\mathrm{B}$, distance $1 \mathrm{~m} \ldots \ldots \ldots$

8. Actual versus predicted moisture values, radial $A$, distance $3 \mathrm{~m} \ldots \ldots \ldots$

9. Aciual versus predicted moisture values, radial $\mathrm{A}$, distance $5 \mathrm{~m} \ldots \ldots \ldots$ 


\section{Application of Neural Networks to Waste Site Screening}

\section{INTRODUCTION}

Waste site screening requires knowledge of the actual concentrations of hazardous materials and rates of flow around and below the site with time. The present approach to site screening consists primarily of drilling boreholes near contaminated sites and chemically analyzing the extracted physical samples and processing the data. In addition, hydraulic and geochemical soil properties are obtained so that numerical simulation models can be used to interpret and extrapolate the field data.

Drilling and physical sample analyses are expensive. Furthermore, the standard numerical simulations can also be costly, both in labor and computer time. Current techniques use finite difference or finite element methods to solve the convective dispersion equations that govern the transport of hazardous wastes. The highly nonlinear equations associated with the saturated/unsaturated soils are particularly difficult to solve, requiring significant computer resources and expertise to obtain a defensible result. Spatial and temporal variations in data describing the subsurface input parameters place further demands on these simulation calculations.

Collection and processing of the data are required to interpolate between points and times and to extrapolate to other distant points and future times. The less precise the data processing methods used the more physical data must be collected to calibrate the numerical model. Conversely, if the modeling techniques are better, less physical data need to be collected. Thus, there is a trade-off between the cost of data collection and the cost of development of better numerical model.

The objective of this work is to investigate the feasibility of using neural network techniques to reduce the cost of waste site screening. A successful technique may lead to an ability to reduce the number of boreholes and number of samples analyzed from each borehole to properly screen the waste site. The analytic tool development described here is inexpensive because it makes use of neural network techniques that can interpolate rapidly and "learn" how to analyze data rather than having to be explicitly programmed.

In the following sections, data collection and analyses will be described, followed by a section on different neural network techniques utilized. The results will be presented and compared with mathematical flow prediction models. Finally, the last section will summarize the research work performed and make several recommendations for future work. 


\section{DATA COLLECTION}

The first step in data collection was to determine what data were available and evaluate it for use in the project. A site was selected after a meeting and discussions with Westinghouse Hanford Company (WHC) scientists. The data are from a site located in the 200 East area of the Hanford/Purex waste disposal reservation. These data were used by Sisson and Lu for field calibrations of their mathematical flow prediction model. ${ }^{1}$ Several utility programs were written to translate the data into convenient computer program format. The list of these programs is given in Appendix A.

A system consisting of a central injection well surrounded by an array of 32 observation wells was constructed at the site. There were four observation wells in each radial direction with eight radial positions equally spaced (see Figure 1).

Over the course of 4 months, 11 injections of approximately 4,000 L of water each were made into the central injection well. The first 10 injections were made at weekly intervals while the 11 th injection was made at the 18 th week. Moisture measurements were obtained by lowering a neutron-neutron moisture meter into an observation well to a specified depth and recording a value in counts/seconds for 15 seconds. This value was then converted into the percentage of water by volume (moisture concentration). A minimum number of measurements that were sufficient to capture the extent of the moisture plume were made. Therefore, these measurements were restricted to the central core of soil immediately surrounding the injection site. This core extends out to a distance of $5 \mathrm{~m}$ radially (at four radial positions, the observation wells are in 2 - $\mathrm{m}$ increments starting at $1 . \mathrm{m}$ radius. At the other four radial positions, they are in $2-\mathrm{m}$ increments starting at a $2-\mathrm{m}$ radius) and from a depth of $3.0 \mathrm{~m}$ to $18.0 \mathrm{~m}$ vertically (in $0.3-\mathrm{m}$ increments).

Sisson and $\mathrm{Lu}^{1}$ observed a strong dependence between the initial moisture content and soil hydraulic properties. For this reason, they used four soil types in their flow prediction modeling. The hydraulic properties for these soil types were estimated from field and laboratory data obtained at sites approximately 5 miles from the test site. 


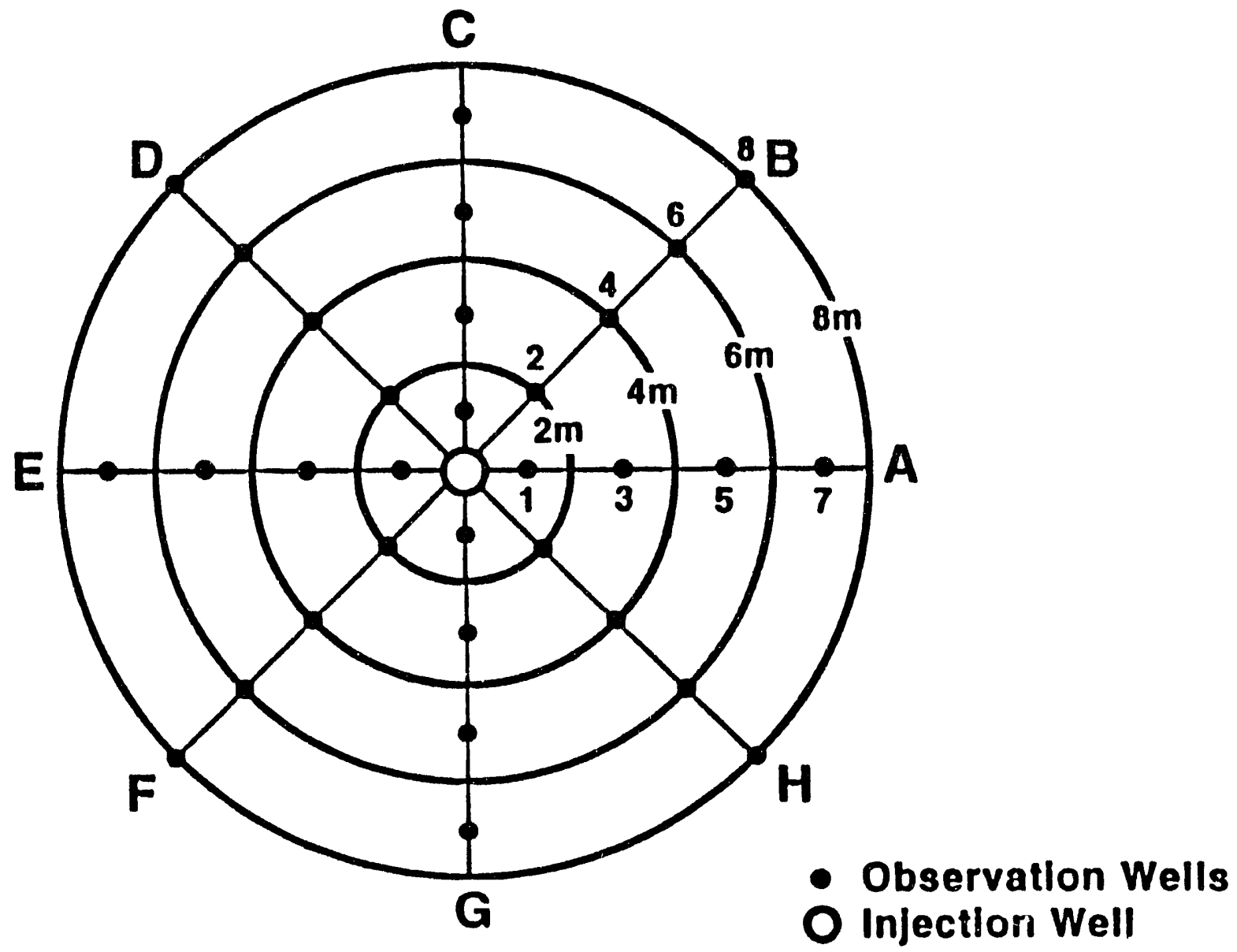

Figure 1. Injection and observation wells layout. 


\section{ANALYSIS OF DATA}

Analyses of the measured data revealed that the flow is not symmetrical. This could be attributed to numerous causes, including slanted soil stratification. Looking at the data with respect to depth, the soil is clearly stratified. This stratification has an effect on the flow of moisture within the system.

The data are very sparse at further distances from the injecrion site, and the amplitude of the moisture plume peak decreases with distance from the injection site. The sparsity of the data is attributed to the fact that only a minimum number of measurements at further distances were performed to ensure that the extent of the moisture plume was captured. The following variables were available for input to the neural network:

- Radial direction

- Radial distance from injection site

- Depth below injection site

- Time since injection start

- $\quad$ Time since injection end

- Moisture level at each point as function of time after injection

- Soil hydraulic properties. 


\section{NEURAL NETWORKS TECHNIQUES}

A neural network is an interconnected collection of "units" that behave similar to neurons in the brain. Each unit receives multiple normalized input signals (activations) and generates a single output signal. The output from each unit is typically connected as inputs to other units in the network. The connections are weighted so that the output of a unit is the weighted sum of the activation signals passed through a nonlinear threshold function. A subset of the units receives input from external sources, and another subset of units generates output for the network, so that the entire neural network can be viewed as a nonlinear mapping between a vector of input activations to a vector of output values.

The connection weights among the units represent the nonlinear mapping function for the neural network. The weights are determined through a process called training, where the weights are incrementally adjusted over multiple training cycles. For the supervised learning techniques used in this report, a training cycle consists of applying an input activation vector and propagating the signals through the network to generate an output value vector. For training, the output vector is compared to a desired target output vector, and the errors are back propagated through the network to adjust the weights. This is repeated until the errors at the output are reduced to an arbitrary small amount.

Once the neural network has been trained, it is capable of recalling the mapping by simply feeding the input activation forward through the network to the output. The neural network is capable of generalizing the mapping so that accurate results can be obtained for input patterns on which it was not specifically trained. In practice, the success of the training and its subsequent application to a problem depends upon constructing training sets of input and target activation vectors that are representative of the problem domain being mapped. Often, because the mapping is nonlinear or the data have not been sufficiently analyzed, the formation of the training sets is more art than science. However, the application of neural networks to a problem will lead to a better understanding of the relational properties of the data and appreciation of the holistic character of the system being observed.

In the case of the waste site screening, the basic physics of migration of "liquid" waste through soil is known indicating strong correlation among the cells defining the site. Because of the inherent hazards at the site, the ability to fully instrument the site to support the physical model is difficult at best. Therefore, the problem addressed by the neural network is to determine whether satisfactory models of the site can be constructed from a more sparse set of measurements.

The neural network approach has potentially two advantages over analytical models. First, the neural network attempts to capture the large scale behavior of the process and progresses to the fine scale behavior if the information is available. Analytical models are typically constructed bottom up extending the micro model (usually linear) in steps to a macro model description of the site. The second advantage is once a neural network has successfully been applied at one site, the same process can be used to screen other sites. In other words, the neural network approach is adaptable and can be "tuned" to a specific site.

2

The approach for development of a tool suitable for site screening using the neural network technique is presented in Figure 2. After suitable processing the data set is divided into a training 


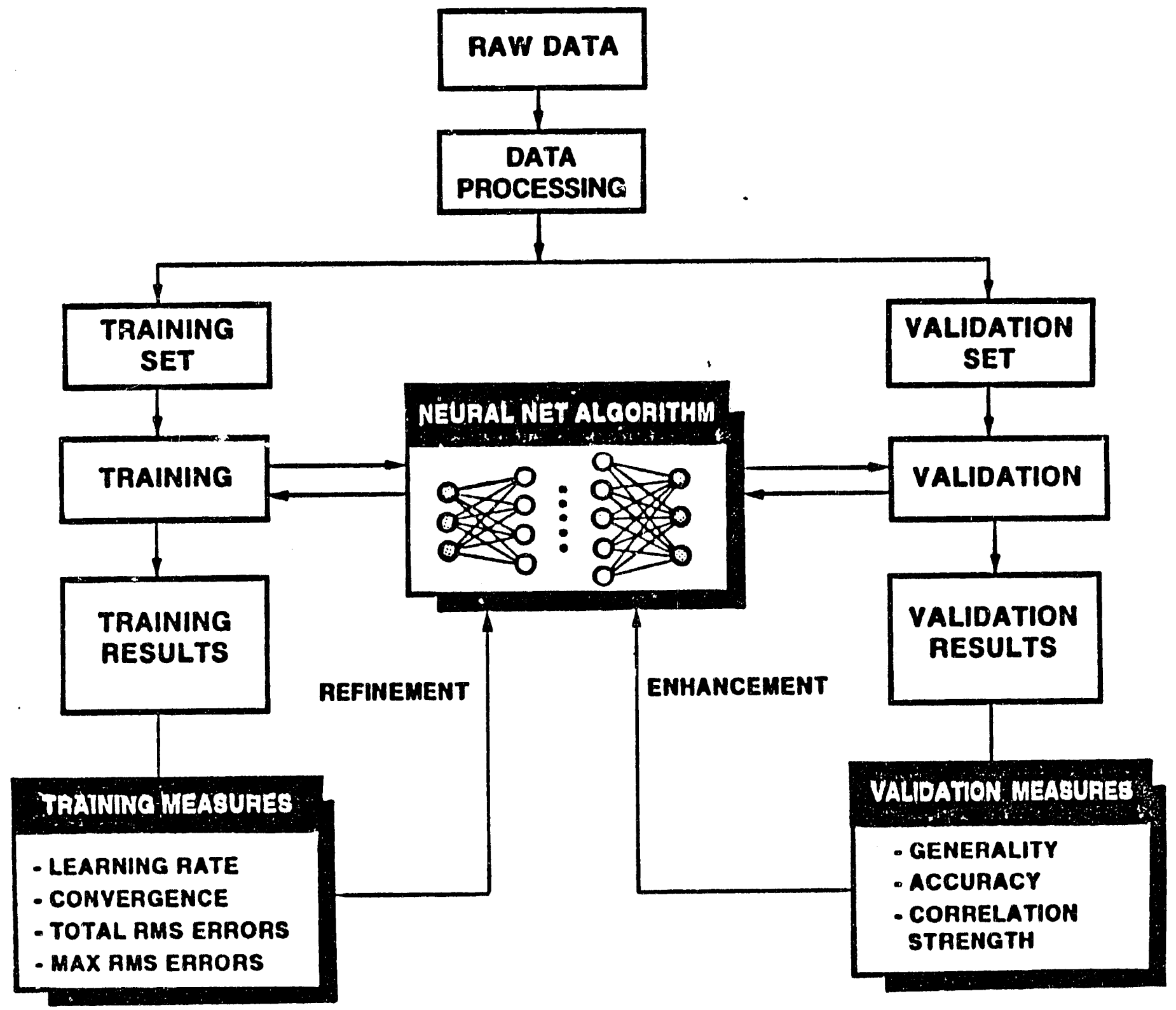

Figure 2. Neural network algorithm application process. 
data set and a validation data set. The neural network is trained by satisfying certain training measures, as shown in Figure 2. The trained network is then validated with the validation data set. This validation should satisfy some specific validation measures as indicated in Figure 2 . The training process is refined until the desired validation measures are satisfied.

The types of units, connection topology among the units, propagation of signals through the network, and learning algorithm are defined by the selected neural network paradigm. For this report two different paradigms, back propagation and fully recurrent neural networks, were utilized to investigate their applicability to waste site screening. Each technique will be briefly discussed below.

\subsection{Back Propagation}

The back propagation network ${ }^{2}$ is an effective mapping function if the relationship between the input activation vector and output activation vector is a continuous nonlinear mapping. Applying the network to data indicates the degree of direct correlation between the input and output vector values. This methodology was used to evaluate the moisture data collected at the test site. Various networks were developed using subsets of the data to determine the completeness of the data and its general application to site screening.

\subsection{Fully Recurrent}

The fully recurrent network ${ }^{3}$ effectively maps multiple time series sequences at the input to one or more output sequences at the output. The units are fully connected within the network so that each unit receives the output from all other units as well as the input activation vector as shown in Figure 3. To achieve an accurate prediction of moisture content as a sequence over time, the collection of input and output activation must be strongly correlated and representative of the time series behavio (patterns) of the system. The combination of sequences represent a closed set of processes defining the site. In other words, each sequence can be derived from some continuous nonlinear combination of the other sequences in the set. The resulting connection weights represent the correlation strengths or codependencies among the sensor measurements. Since the present data are sparse, a three-dimensional model, which would be the most useful and accurate model, could not be trained to any reasonable precision. A two-dimer.sional model, however, was a good approximation. The effective moisture values for the two-dimensional model were obtained at each depth from the surface and distance from the injection site. This reduced the space to two dimensions, depth and distance from the source. 


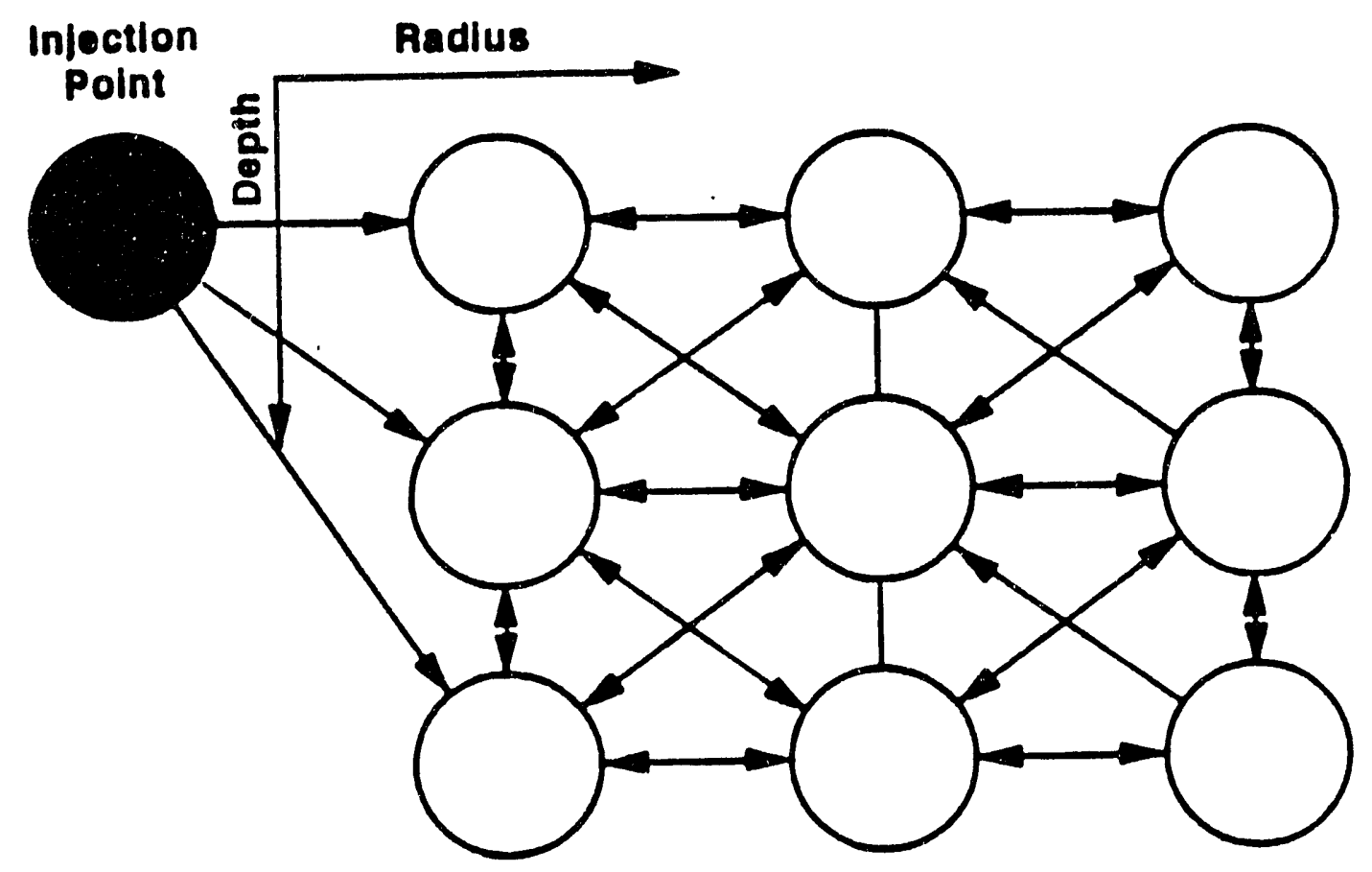

Figure 3. Fully recurrent network. 


\section{RESULTS}

\subsection{Back Propagation}

The initial set of networks used only the moisture values in the neighboring cells to predict the moisture value in the test cell. In this case, the back propagation network is learning how moisture content in neighboring cells at time, $t$, directly maps to the moisture content in the test cell at time $t+1$. This technique assumes that the relationship is independent of time. At any arbitrary time, $t$, the neural network should accurately determine the moisture content at time, $t+1$, regardless of the previous moisture history of the neighboring cells or the test cell. The initial networks could predict moisture at a specific test cell but had difficulty generalizing the learning to other sites using the same learned network weights. This is understandable, since other measured parameters. such as soil conductivity, are required to screen a site.

When soil type, related to soil conductivity, was added as a reference input, the back propagation network was able to generalize the connection weights to other cells not included in the initial training set. The training set required samples distributed over various soil types, injection events, and injection times to generate predictions within $10 \%$ of actual measurements. These results indicated that there was significant correlation in the collected data but did not support the objective of screening a site with reduced data collection. The training required data collected throughout the site and still required time since last injection as an input.

Time is a problematic measurement in screening a site. Except for controlled experiments, the injection time and rate of injection are not known. Even in the controlled experiments, the sampling time between measurements may vary and not be sufficient to capture significant events such as the peak moisture at a cell or initial start of a rise in moisture content. Through analyses of the back propagation network performance, time since last injection was used primarily to differentiate periods of increasing or decreasing moisture. To remove direct dependency on the time, coarse shaping parameters were introduced. The shaping parameters indicated the direction of the moisture change over the previous three measurements. These allowed the network to bifurcate the predictions for rising and falling moisture content and improved accuracy in predicting peak moisture following an injection.

The best results obtained used a network with input moistures and soil type for each neighboring cell and three input shaping parameters feeding to five hidden units that resulted in one prediction output for the moisture content of the test cell as shown in Figure 4. During training, the average root mean square (RMS) error was $0.78 \%$ with a maximum output error of $5 \%$. During validation recall, the average and maximum errors were $1.1 \%$ and $6 \%$, respectively, indicating acceptable generalization. (This level of accuracy seems to be adequate for site screening.) The size of the sample was about 700 of which $90 \%$ was used for training, and the remaining $10 \%$ was used during validation. These results are well within the requirements of accurate site screening. However, the primary problem is that the resulting neural network cannot be applied to other sites and still requires extensive sampling of the new site to adapt it to that site. It does not reduce the number of core samples that must be collected. The back propagation network process development is fully described in Appendix B. 


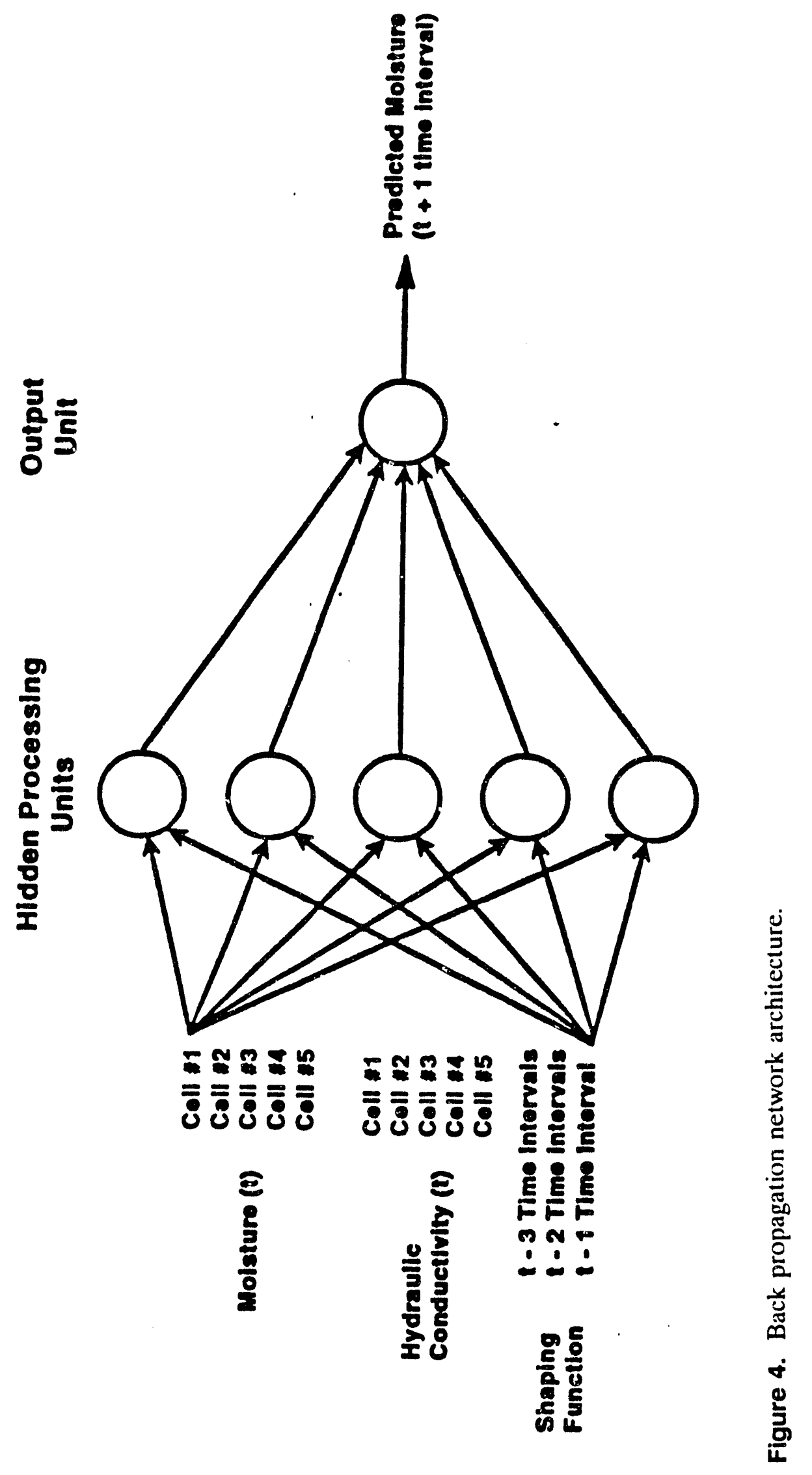




\subsection{Fully Recurrent Network}

The back propagation network results indicate that the combination of moisture content, soil characteristics, and previous moisture history are sufficient to describe the dynamics at a site. The advantage of the fully recurrent network over feed forward networks (such 25 the back propagation network) is that the recurrent network is learning a set of recurrent maprjing equations rather than direct mapping functions. The fully recurrent network is therefore at/e to incorporate previous history in the screening of the site. Although the data must be presented as a time sequence, the network can generalize over varying sample rates and effectively become independent of specific time and sample rates to screen the site.

Figure 5 presents the fully recurrent network architecture using the same measurement data used in developing the back propagation networks. In an array of nine cells, moisture values of three cells are used as input to predict the moisture values of six cells at different levels. This will be called the three- to six-setup configuration. Because data were collected more extensively vith depth than with radius from the injection site, the network was set up to predict the moisture content with depth. The same approach could be used to screen the site radially from the injection poini, reducing the number of bore sites required to screen the site.

The networks were trained with inputs from six different depths $(540,600,660,750,810$, and $900 \mathrm{~cm}$ ) and predicted the moisture content for cells at +30 and $+60 \mathrm{~cm}$ from the input depth. The various networks were trained until the total RMS error achieved was $0.5 \%$ ( -3400 training cycles). During training the output from the network was replaced by the actual measurement values before recycling for the next output. This is referred to as forced training. During recall validation processing, the output from the neural network is recycled (called free recall) so that the network only receives the initial state for all cells and the input moisture value for the three input cells for each subsequent time step. This tests the stability of the network to errors in the output over extended times.

Figure 6 gives the results of the moisture values for the first output cell at $570 \mathrm{~cm}$ depth at the $1 \mathrm{~m}$ radial given the moisture values at the $540 \mathrm{~cm}$ depth. The network was trained on only the data in the third injection and validated over all of the five injections. The total number of data set for training on the third injection was about 234 , which represented $20 \%$ of the total data set for five injections. The figure shows a comparison of the actual measurements, neural network predictions, and mathematical model predictions for the cell. Figure 7 shows similar results for the same depth but along a different radial from the injection site. As can be seen, the results of the neural network are comparable to the results of the mathematical model. The degree of agreement indicates that the neural network is capturing the same dynamics incorporated in the mathematical model. The ability of the network to predict moisture over multiple injections indicates stability of the network to output error, which can be credited to the strength of the correlation among the cell measurements. In fact, predictions remain accurate $(1 \%$ RMS $\times 5 \%$ maximum error) for the different cells even when the predominate injection peak is not present (see Figures 8 and 9) at larger radial distances. The fully recurrent network process development is described in detail in Appendix C. 


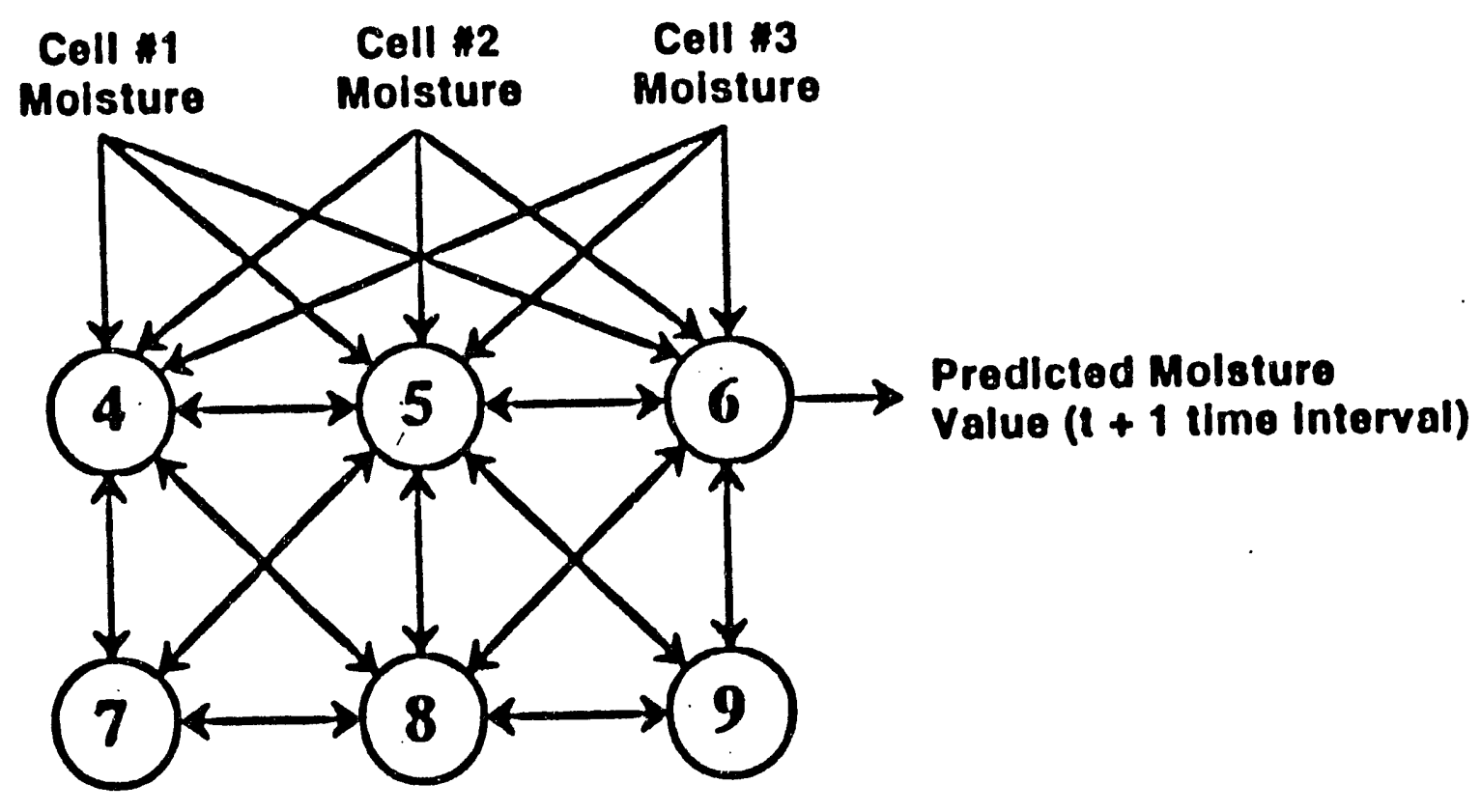

Figure 5. Fully recurrent network architecture (three to six matrix). 


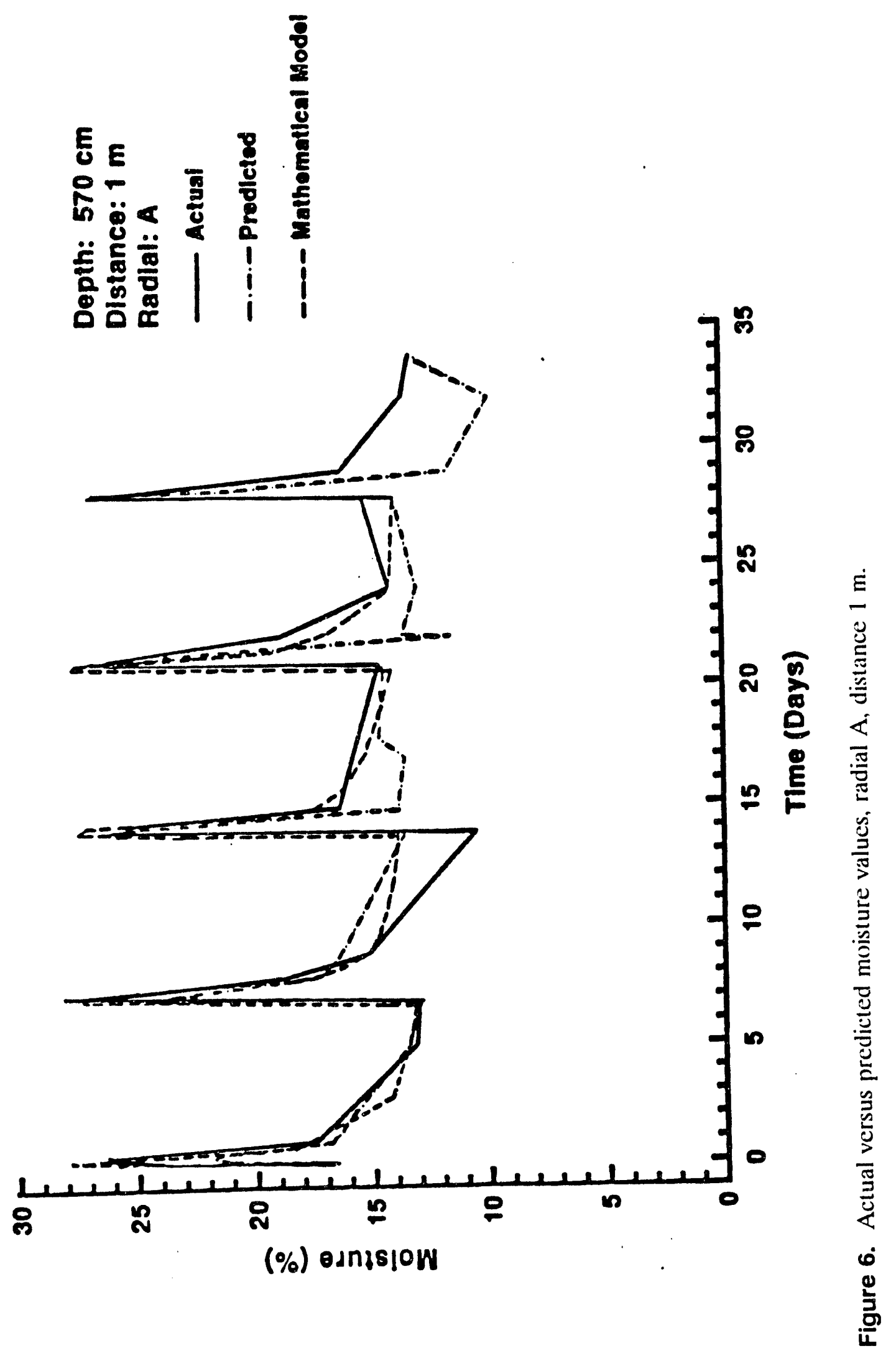




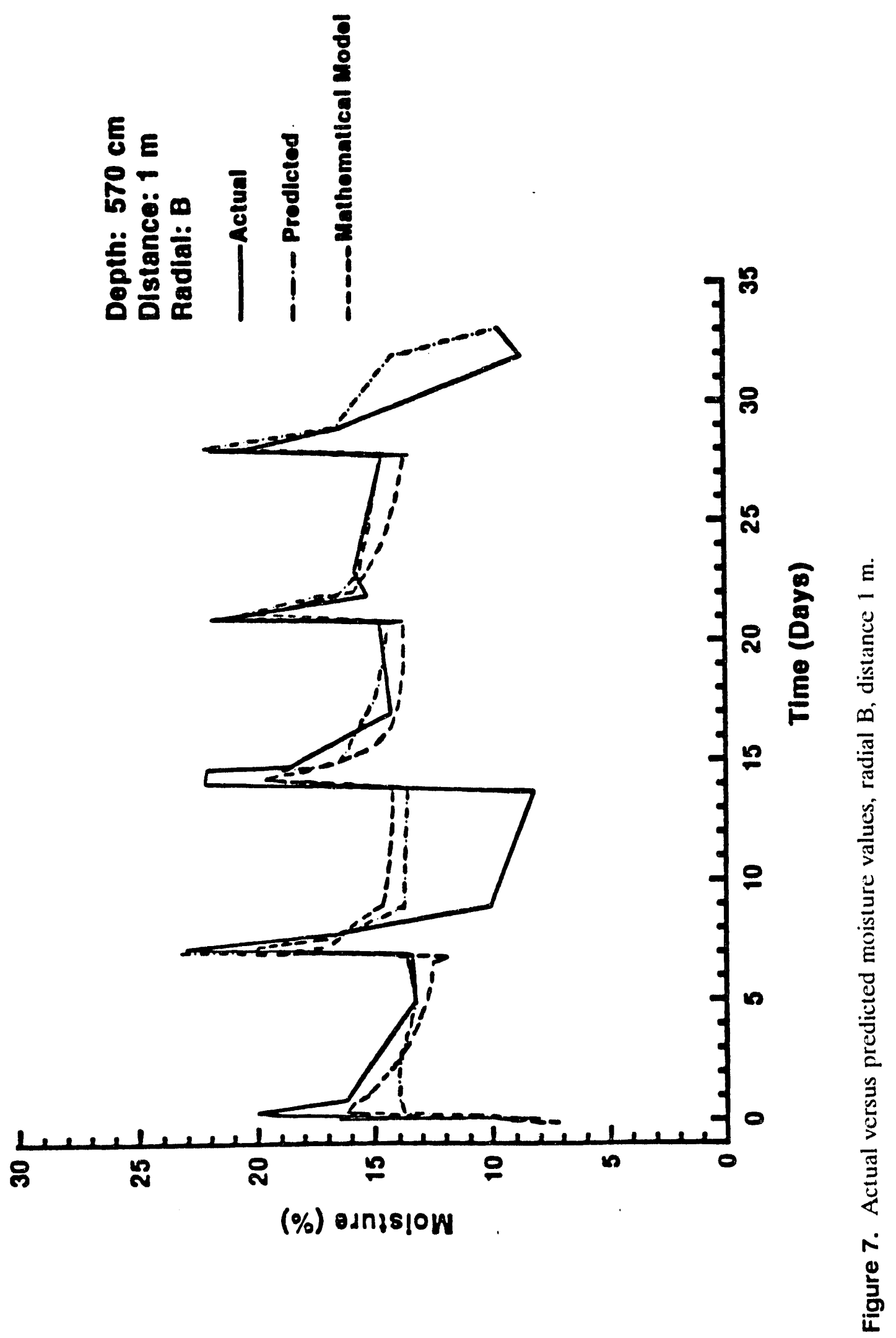




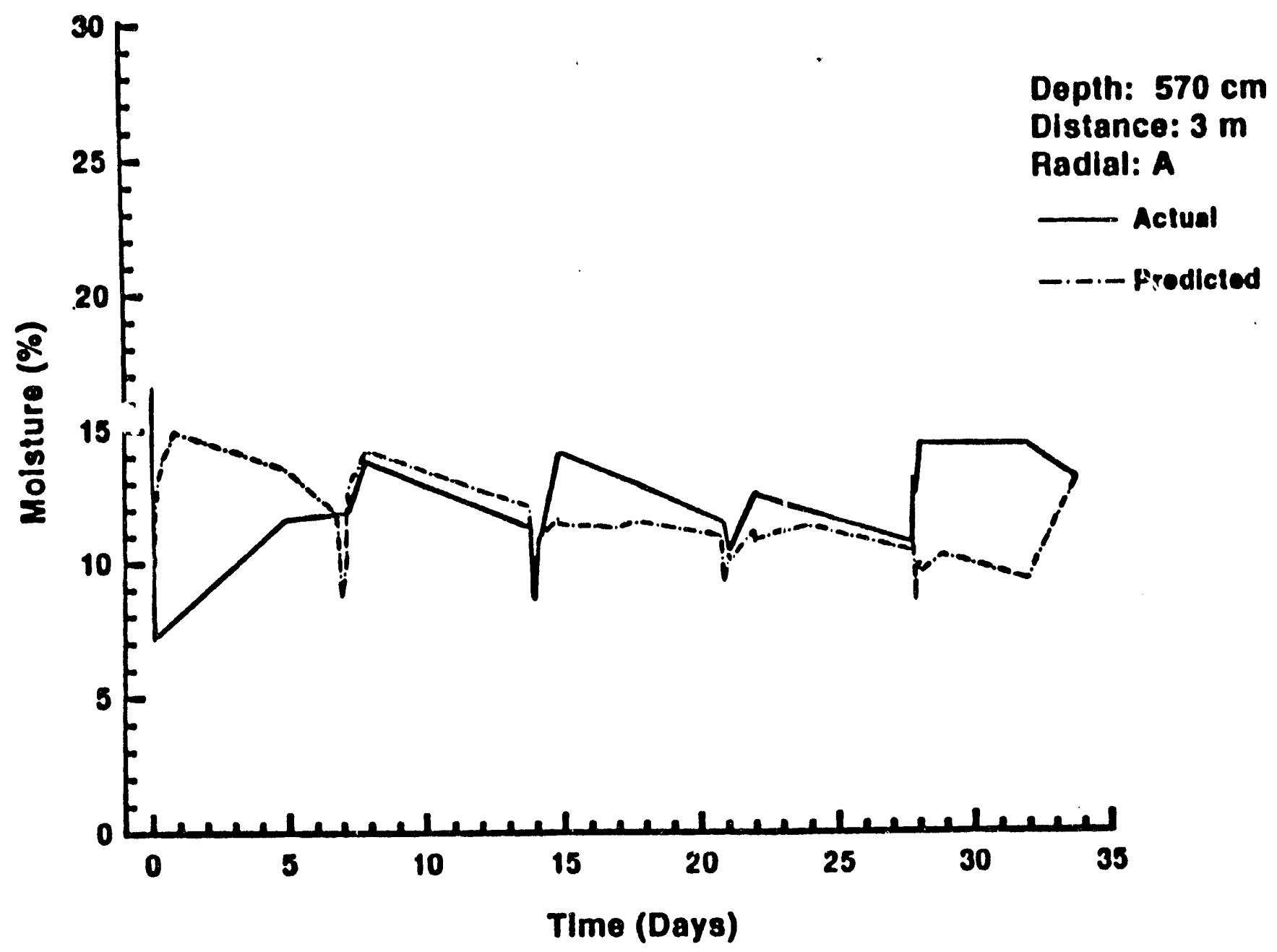

Figure 8. Actual versus predicted moisture values, radial $\mathrm{A}$, distance $3 \mathrm{~m}$. 


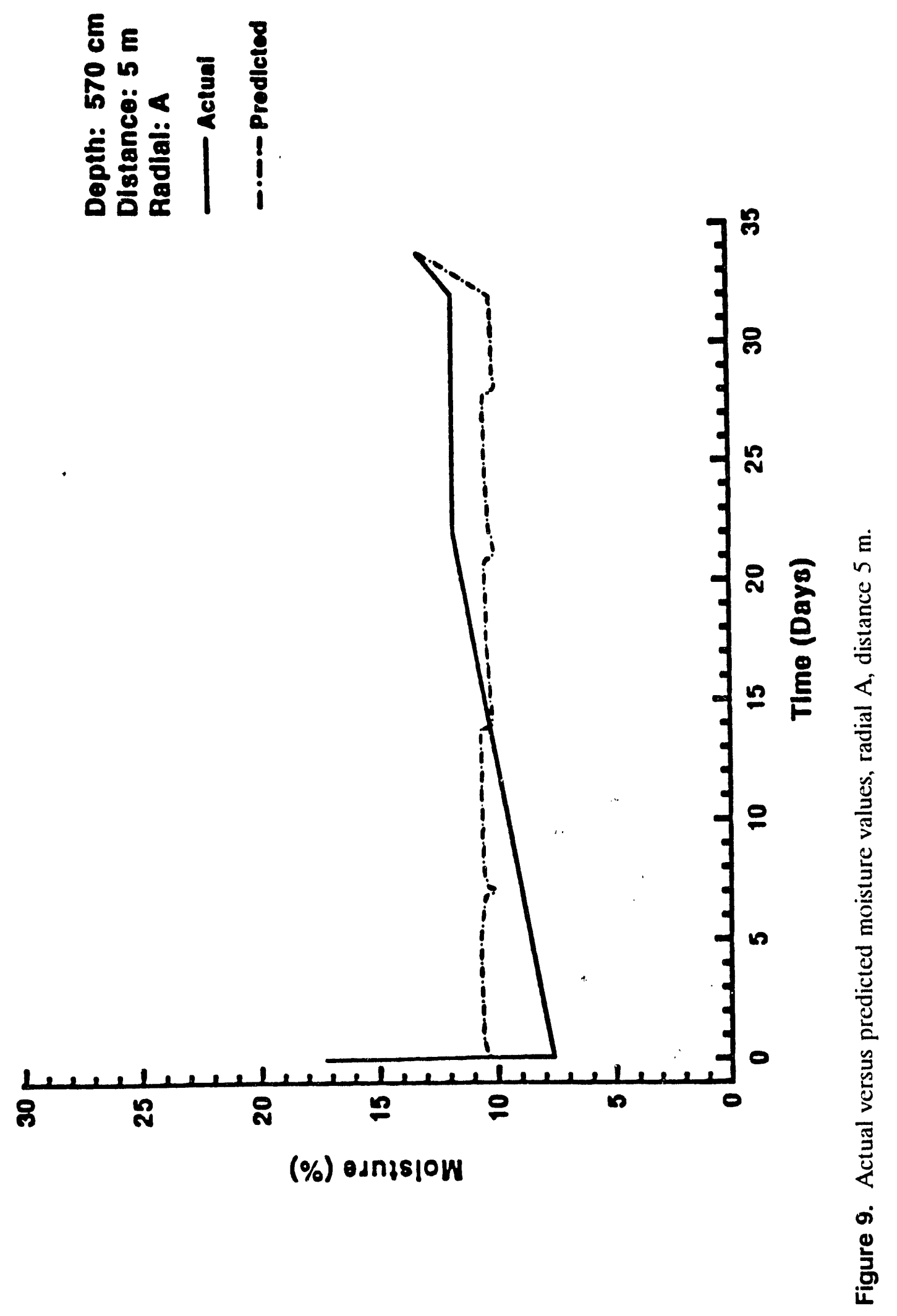




\section{DISCUSSION OF RESULTS}

The differences between the neural network prediction and actual measurement, referred to as innovations, represent information not captured by the neural network during training. The innovations or deviations from modeled behavior can be due to a number of causes including systematic measurement error, such as under sampling and measurement calibration or bias errors; particular soil conditions, such as rock stratification or soil moisture conduits; or processes not observed in the original training samples. Because the neural network has been derived independent of the mathematical model, the network in this configuration can be used to validate the original data measurements or suggest novelty from normal learned behavior.

The connection weights for a fully connected recurrent back propagation netwcik have several features that differentiate it from normal back propagation. The connection weights represent the correlation strength or codependency among the input and output measurements. Unlike back propagation net-vorks, the weights are well ordered so that the same weights can be derived from different initial states and even different training data for the same process. Therefore, the weights represent the combination of the nominal model for the site independent of specific soil conditions and a local model, which changes the weights to account for conditions specific to the cell neighborhood being predicted. This suggests that there is a relationship between soil conditions and the connection weights and that it may be possible to change the weights to adapt the recurrent network to the new site without extensive training for the new site. This observation is the basis for a general adaptive tool for waste site screening.

The results of the application of the fully recurrent neural network to site data are promising for a general purpose site screening tool. To date, recurrent neural networks have been developed that are able to capture the dynamics at different depths and radii from the injection site; to generalize to different radials; to account for local variations in soil conditions; and are independent of injection time and rate. In its current configuration, the recurrent network reduces the number of test bore sites by extending the accuracy of the predictions over more widely spaced test sites. 


\section{APPLICATIONS}

There is a variety of applications of this technique in environmental site screening and remediation to reduce both the cost of drilling and sampling. Following are some examples that demonstrate how this technique could be applied to some of the present environmental problems.

\subsection{Optimum Well Siting}

A major factor in the success or failure of a site characterization and cleanup is the effectiveness of the well siting and the sampling procedures used. While the need is obvious, the cost entailed in doing extensive drilling and sampling will be prohibitive and will force the position of secking the most information from the fewest samples.

Traditional methods of drilling and sampling are based on classical statistical methodology where the points are selected randomly in the hope that the contaminated positions will be effectively characterized by the sampling.

Sampling design can be substantially improved by one of the following methods:

1. A model that takes into account the underlying physical process, such as transport, flow, reaction, etc. This model-aided sampling approach is an improvement over the more traditional approaches because it uses a model of the actual process driving the contaminants to their current and future states. The problem of this method is that it can also be costly, both in labor and computer time. In addition, the spatial and temporal variations in data describing the subsurface, tlow, and other physical parameters place further demands on these modelings.

2. A neural network model that could be trained for the existing sampling data obtained from existing bore sites. This trained network could be used to decide where the next well should be drilled. The neural network can predict the waste contaminants in locations near the wells that have already been characterized. The waste level map obtained for this exercise will determine the optimum location of the next hole to be drilled. Obviously, there is no need to drill at the locations where there is not any major change of contaminants with respect to other characterized locations.

\subsection{Reduction of Number of Sampling}

The results indicate that the fully recurrent network approach has the potential to predict waste contamination level at certain depth from the waste contamination levels measured at more shallow depths. The three- to six-setup configuration of fully recurrent network shown in Figure 5 can be utilized for this purpose. The neural network will be trained from the existing sampling data obtained from the existing neighboring bore sites. Assuming minor variations in geology of neighboring bore sites, one could use the trained network and three- to six-setup configuration to predict the waste contamination levels at the next two levels down (if the geology of the two sites are different, it is possible to change the connection weights of the fully recurrent network to adapt it to the new site without extensive training for the new site). The new values could be utilized to predict the waste 
levels at even lower depth, and this process could continue. At some point in the process, the waste contamination level can be measured at a certain depth to check the accuracy of the predictions. This process can substantially reduce the number of samplings.

\subsection{Reduction of Number of Bore Sites}

The trained network mentioned above could also predict the contamination levels at the next two bore sites out in the radial direction with the three- to six-setup configuration. These new values could be used to predict the contamination levels at even larger radii. At some point, the contamination levels can be measured to check the accuracy of the predictions. This process can substantially reduce the number of bore sites. 


\section{CONCLUSIONS AND RECOMMENDATIONS}

The present approach to site screening is expensive and time consuming. The feasibility of using neural network techniques to reduce the cost of waste site screening was investigated. The networks were trained with data received from WHC. The results indicate that the network trained with the fully recurrent technique show satisfactory generalization capability. The predicted results are close to results obtained from mathematical flow prediction model. It is possible to develop a new tool to predict the waste plume, thus substantially reducing the number of the bore sites and samplings.

Several applications have already been identified for this technique. Optimum well siting would be an obvious one, where substantial saving could be achieved for a proper site characterization. This technique is simple, straightforward, and inexpensive. It is recommended that this technique be applied to one of the environmental sites that has already been characterized to evaluate the validity of the technique. Once validation results proved to be satisfactory, the technique may then be used at sites that are presently being characterized to reduce the cost of screening and characterization by substantiai amount. 


\section{REFERENCES}

1. J. B. Sisson and A. H. Lu, Field Calibration of Computer Models for Application to Buried Liquid Discharges: A Status Report, Rockwell International, RHO-ST-46, August 1984.

2. D. E. Rumelhart, G. E. Hinton, and R. J. Williams [D. E. Rumelhart and J. L. McClelland, (eds.)] "Learning Internal Representations by Error Propagation," Parallel Distributed Processing: Exploration in the Microstructure of Cognition, 1, Massachusetts Institute of Technology Press, Cambridge, Massachusetts, 1986, pp. 318-362.

3. R. J. Williams and D. Zipset, "A Learning Algorithm for Continually Running Fully Recurrent Networks," Neural Computation, 1, 1989, pp. 270-280. 
Appendix A

Computer Source Programs Listing 
A-2 


\section{Appendix A}

\section{Computer Source Programs Listing}

This appendix lists all the source programs developed during the course of the project to make appropriate files for various purposes. Figure A-1 shows the flow chart, starting with the raw data given to Science Applications International Corporation by Westinghouse Hanford Company and ending with moisture prediction plots. 


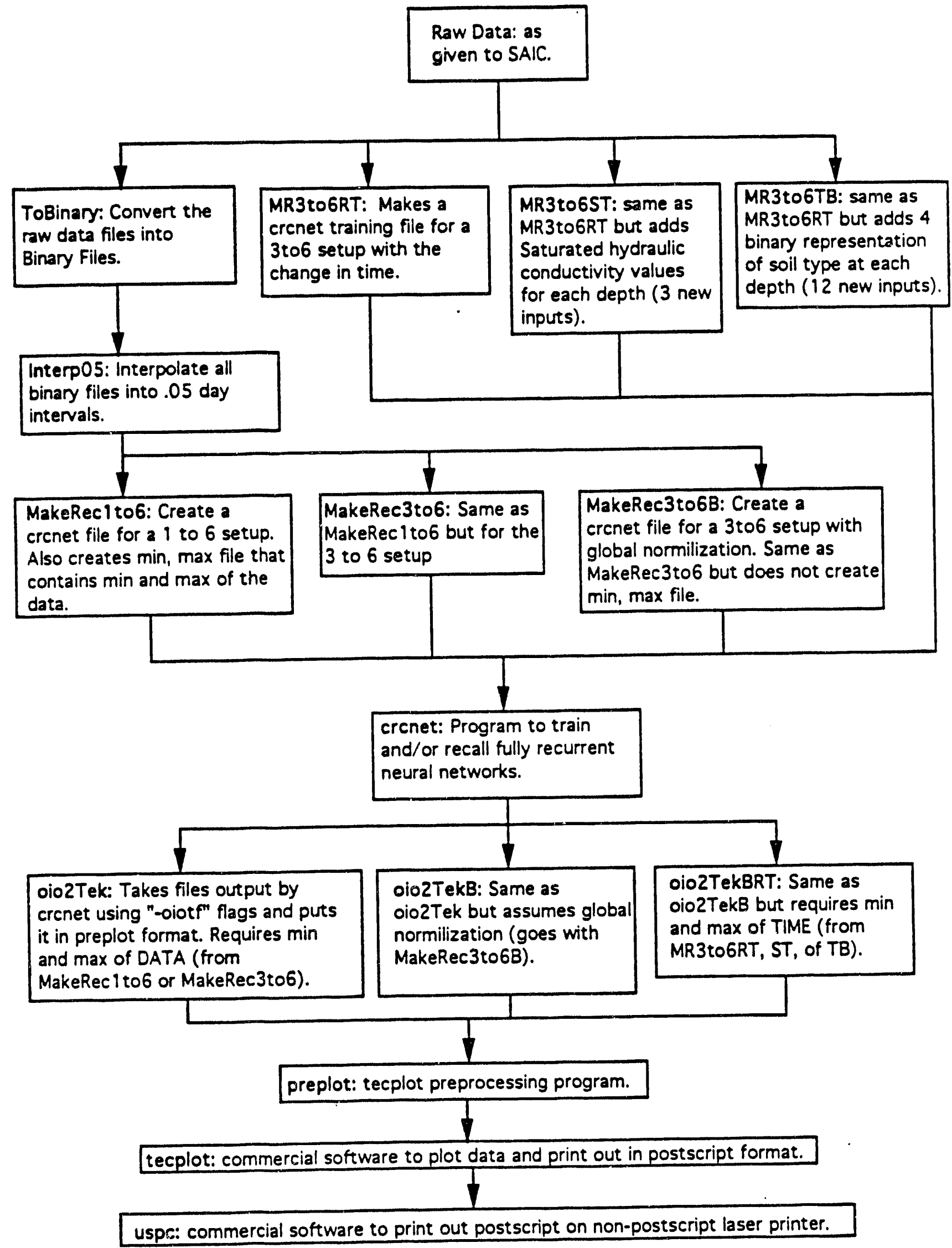

Figure A-1. Recurrent net data flow chart. 


\section{TOBINARY.cpp}

\#include <stdio.h> \#include <sudlib.h>

\#include <math.h>

\#include <iostream.h>

\#define NUM_FILES 32

int main()

1

FILE *pFILE_Input;

FILE * pFILE_Output;

float afTime[73];

float afMoisture[73];

int iRow_loop;

int iLoop;

int iFile_loop;

char SBuffer[128];

char asFile_Prefix [32][12] =

1

"a1.","a3.","a5.","a7.",

"b2.","b4.","b6.","b8.",

"c1.","c3.","c5.","c7.",

"d2.","d4.","d6.","d8.",

"e1.","e3.","e5.","e7.",

"f2.","โ4.","โ6.","f8.",

"g1.","g3.","g5.","g7.",

);

"h2.","h4.","h6.","h8."

char SOutput_File_Name[12];

char sInput_File_Name[12];

for (iFile_loop $=0$;iFile_loop $<$ NLiM_FlLES; iFile_loop++)

1 sprintf (sInput_File_Name,"Fcsdat",asFile_Prelix(iFile_loop]);

sprinuf (sOutput_File_Name," "cicsbin",asFile_Prelix[iFile_loop]);

pFILE_Input $=$ fopen $($ sInput_File_Name, $" r " \bar{p})$ :

pFILE_Output = fopen (sOutput_Filc_Name,"wb");

$/ /$ open the files

for (iRow_loop $=0$;iRow_loop $<51 ;$ iRow_loop ++ )

l for (iLoop $=0$; iLoop $<73$; iLoop ++ )

1 fscanf (pFILE_Inpul," "Fs",sBuffer);

afTime[iLoop] $=$ atof(sBuffer);

1) // read in the time stamps

fwrite (afTime, sizcol (afTime), l,pFILE_Output);

]

// output to file

fclose (pFILE_Input);

fclose (pFILE_Output);

$/ /$ close the files

了 


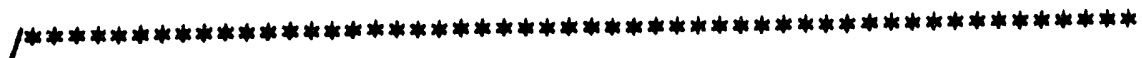

Jeff Hilton

- This file reads in from the raw

data of radius A starting at 1 meter and produces

a data file for crenet for a network of the

configuration:

A B C T

123

456

$\mathrm{T}=$ time

letter $=$ input

numbers $=$ output

- In the this version the min and max are assumed

$\min =3.02$

$\max =30.110001$

The above values are the $\min$ and $\max$ for:

$1,3, \& 5$

A

depths $300 \mathrm{~cm}-1800 \mathrm{~cm}$

injections 1.5

$* * * * * * * * * * * * * * * * * * * * * * * * * * * * * * * * * * * * * * * * * * * * * * * * * * * * 1$

\#include <stdio.h>

\#include <stdlib.h>

\#include <string.h>

\#include "RDGLib.h"

\#define NUMDATA 9

\#define ARRA YSIZE 500

\#define NUMOUTPUTS 6

/*****************************************************

- This routine gets the raw data and its corresponding time.

$* * * * * * * * * * * * * * * * * * * * * * * * * * * * * * * * * * * * * * * * * * * * * * * * * * * * 1$

void

getrawdata (rawdata, timedata, sizeraw, thedepth)

float **rawdata;

float **timedata;

int *sizeraw;

int thedepth;

I

FILE *infilel;

FILE *infile 3 ;

FILE *infile5;

int index1, first_inj, last_inj;

first_inj $=1$;

last_inj $=5$;

infile1 = fopen("a1.dat"," $\pi$ ");

if (infilel $==$ NULL)

$\operatorname{exit}(-1)$;

infile3 = fopen("a3.dat", "rt"); 
fillTimes (timedata[0], sizeraw[0], timedata[1], sizeraw[1],

temptime, \&tempsize);
fillTimes (timedata[2], sizeraw[2], temptime, tempsize, time, sizetime);

for (index 1 = 0; index $1<$ NUMDATA; index $1++$ )

fillData(rawdata[index 1], timedata[index 1], sizeraw[index 1], time, "sizetime);

)

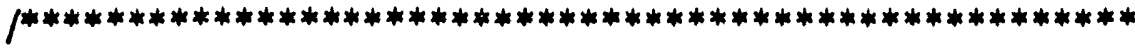

- This routine converts first_inj and last_inj into indexes of the first and last injection times in the array time

- MUST be called befor time is nored or converted to change in time.

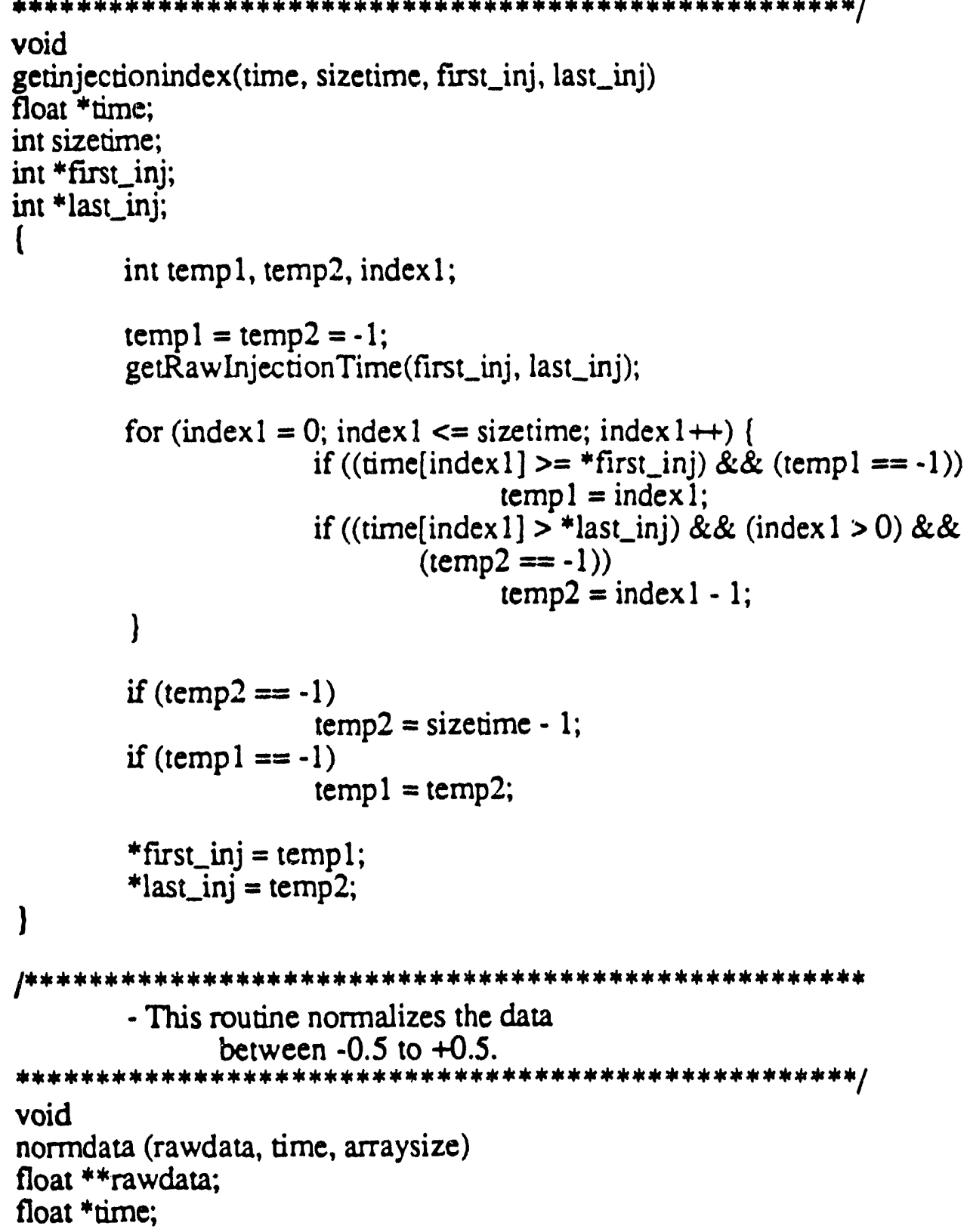

- This routine normalizes the data between -0.5 to +0.5 .

void

normdata (rawdata, time, arraysize)

float **rawdata;

float *time; 
int arraysize;

l

float $\min , \max$;

$\min =3.02$

$\max =30.110001$

normAllData (rawdata, NUMDATA, arraysize, min, max);

$\min =\max =\operatorname{time}[0]$

getMinMax (time, arraysize, \&min, \&max);

normDataNP5ToPP5(ime, arraysize, $\min , \max$ );

printf ("nmin $=\% .6 \mathrm{fn} ", \mathrm{~min})$;

1 printf ("max $=\% .6 \mathrm{fn} ", \max$ );

\footnotetext{
*****************************************************

- This routine takes the data set and reduces it to only the injection times wanted.

*****************************************************/

void

converttowantedinj(rawdata, time, sizetime, first_inj, last_inj)

float **rawdata;

float * time;

int *sizetime;

int first_inj;

int last_inj;

l

int index 1 , index 2 , index 3 ;

*sizetime = last_inj - first_inj + 1;

if (first_inj $>0$ )

for (index $1=0$, index $2=$ first_inj; index $2<=1$ last_inj;
index $1++$, index $2++)($

time[index 1$]=$ time[index2];

rawdata[index 3][index2];

\}

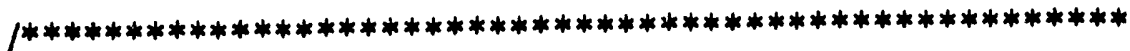

- This routine prints the data out in crenet

format.

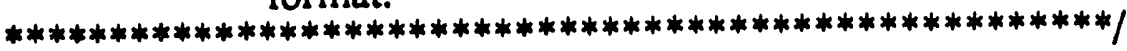

void

outdata (filename, rawdata, time, sizearray)

char *filename;

float **rawdata;

float *time;
}

for (index $3=0$; index $3<$ NUMDATA; index $3++$ )

rawdata[index3][index 1] = 


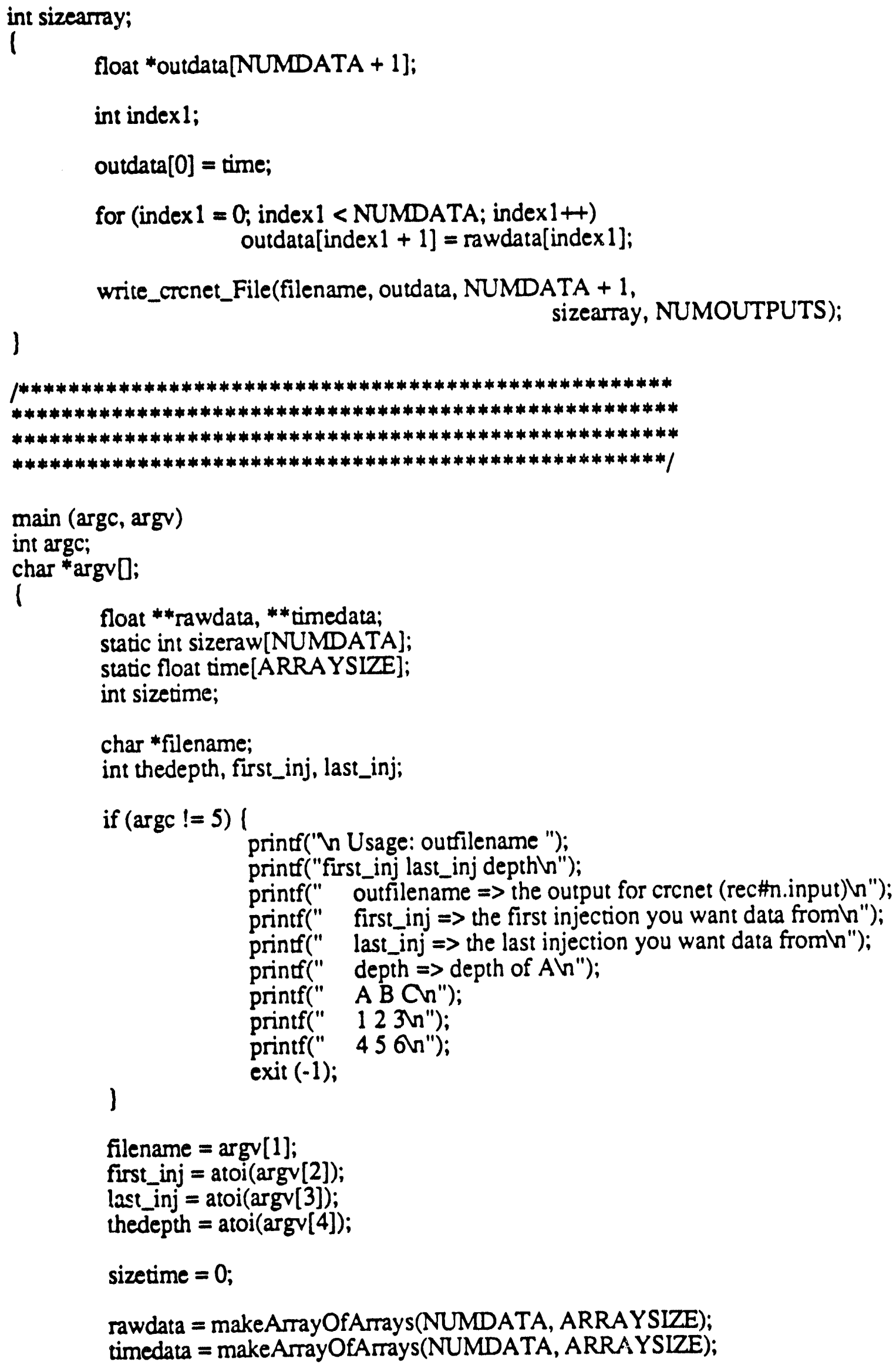


getrawdata(rawdata, timedata, sizeraw, thedepth);

extractdata (rawdata, timedata, sizeraw, time, \&sizetime);

getinjectionindex(time, sizetime, \&first_inj, \&last_inj);

convertoChangeInTime (time, sizetime);

converttowantedinj(rawdata, time, \&sizetime, first_inj, last_inj);

normdata (rawdata, time, sizetime);

outdata (filename, rawdata, time, sizetime);

deallocateArrayOfArrays (rawdata, NUMDATA); deallocateArrayOfArrays (timedata, NUMDATA); 


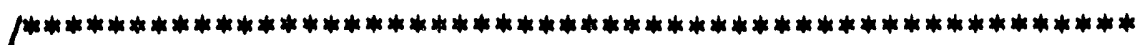

Jeff Hilton

- This file reads in from the raw

data of radius $A$ starting at 1 meter and produces

a data file for crenet for a network of the

configuration:

A B C T

123

456

$\mathrm{T}=$ time

letter $=$ input

numbers = output

- In the this version the min and max are assumed

$\min =3.02$

$\max =30.110001$

The above values are the min and max for:

$1,3, \& 5$

A

depths $300 \mathrm{~cm}-1800 \mathrm{~cm}$

injections $1-5$

****************************************************1

\#include <stdio.h>

\#include <stdlib.h>

\#include <string.h>

\#include "RDGLib.h"

\#define NUMDATA 9

\#define ARRAYSIZE 500

\#define NUMOUTPUTS 6

\#define NUMSOILPROPS $3 /$ * the number of inputs */

f****************************************************

- This routine gets the raw data and its corresponding time.

$* * * * * * * * * * * * * * * * * * * * * * * * * * * * * * * * * * * * * * * * * * * * * * * * * * * *)$

void

getrawdata (rawdata, timedata, sizeraw, thedepth)

float **rawdata;

float **timedata;

int *sizeraw;

int thedepth;

1

FIIE *infile 1;

FILE *infile3;

FILE *infile5;

int index 1, first_inj, last_inj;

füst_inj $=1$;

last_inj $=5$;

infilel = fopen("a1.dat"," $r$ ");

if (infilel $==$ NULL) 


\section{MR3ío6ST.c}

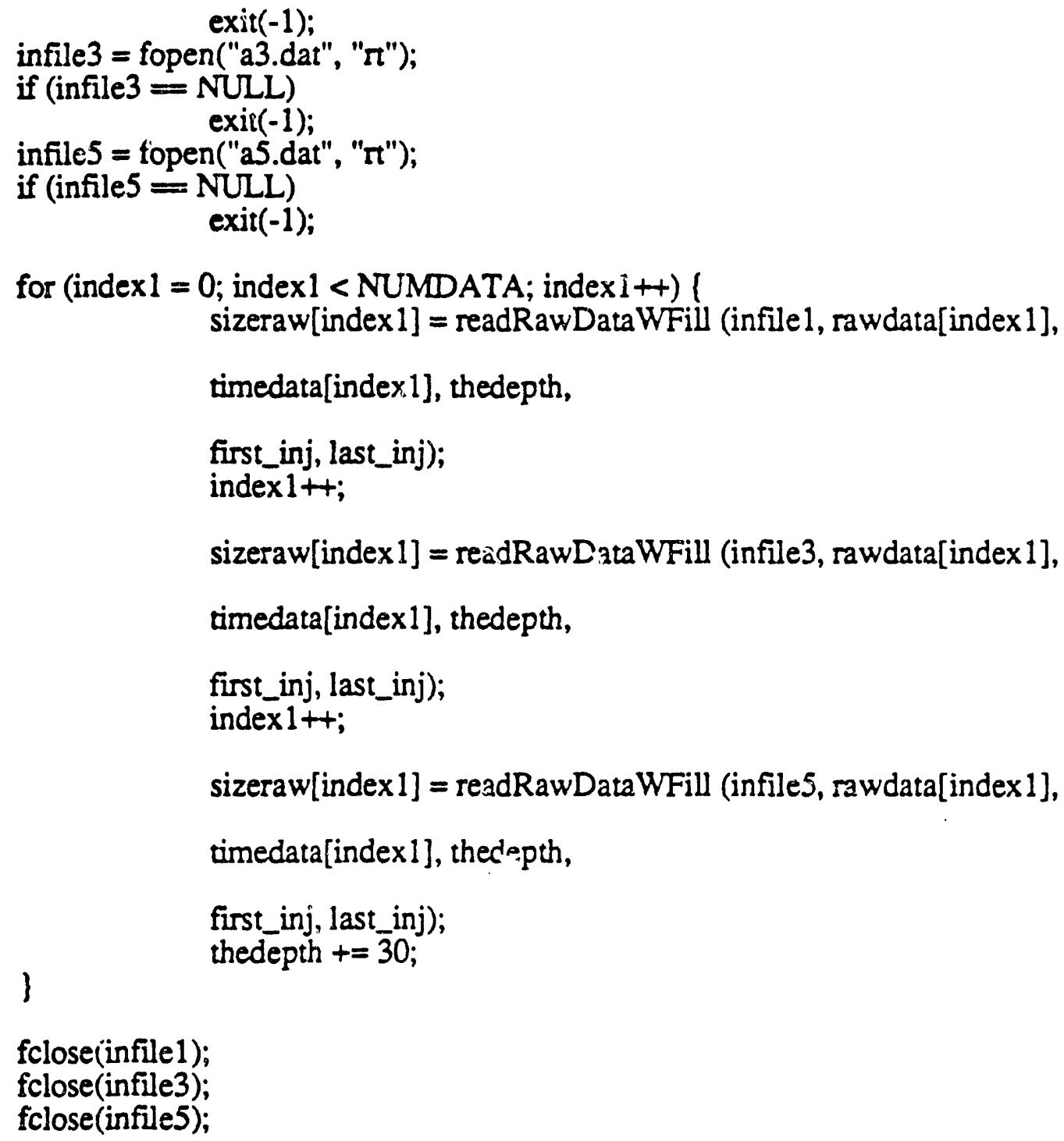

- This routine combines all the possible times

and then interpolates data for those points that do no have data at new times

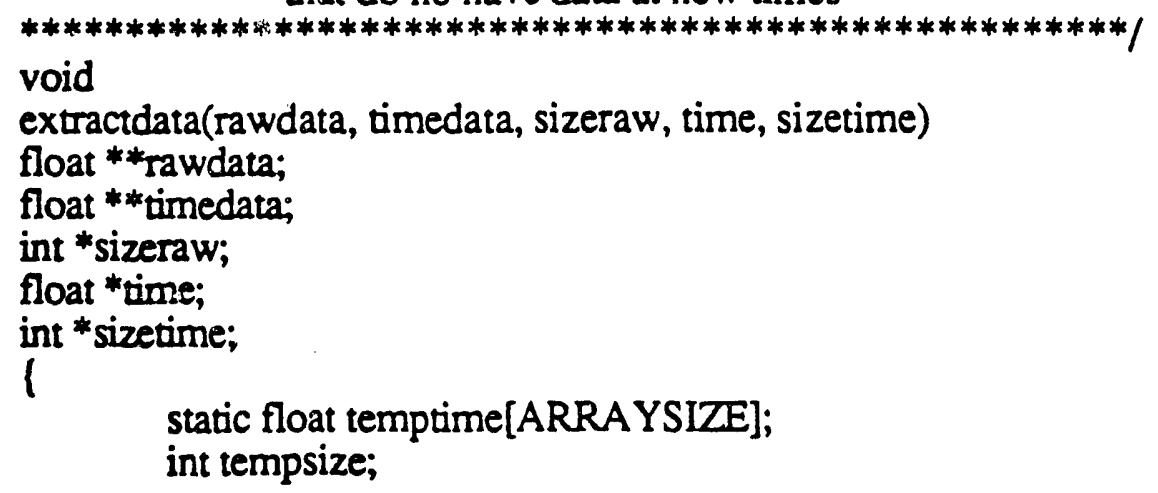




\section{MR3to6ST.c}

int index 1;

fillTimes (timedata[0], sizeraw[0], timedata[1], sizeraw[1], temptime, \&tempsize);

fillTimes (timedata[2], sizeraw[2], temptime, tempsize, time, sizetime);

for (index $1=0$; index $1<$ NUMDATA; index $1++$ )

fillData(rawdata[index1], timedata[index1], sizeraw[index1], time, *sizetime);

\}

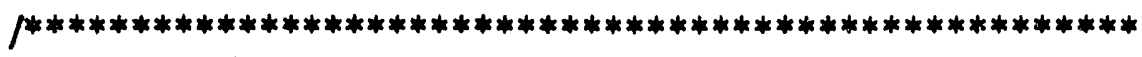

- This routine converts first_inj and last_inj into indexes of the first and last injection times in the array time

- MUST be called befor time is nored or converted to change in time.

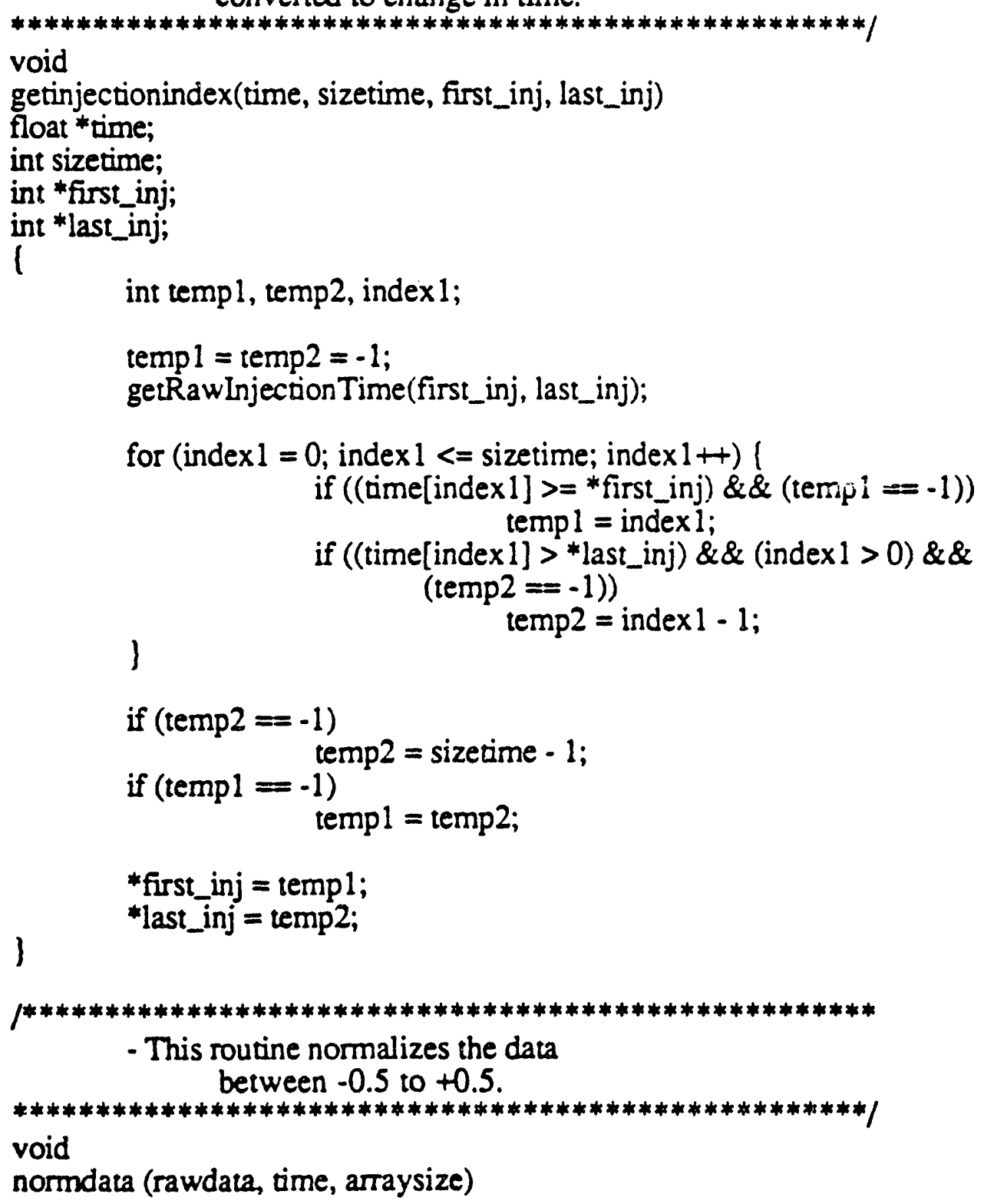

- This routine normalizes the data between -0.5 to +0.5 .

$* * * * * * * * * * * * * * * * * * * * * * * * * * * * * * * * * * * * * * * * * * * * * * * * * * * * 1$

void

normdata (rawdata, time, arraysize) 
float **awdata;

float *time;

int arraysize;

l

float min, max;

$\min =3.02$

$\max =30.110001$

normAllData (rawdata, NUMDATA, arraysize, $\min , \max$ );

$\min =\max =\operatorname{time}[0]$

getMinMax (time, arraysize, \&min, \&max);

normDataNP5ToPP5(time, arraysize, min, max);

printf ("Vnmin = \%.6Nn", $\mathrm{min}$ );

) printf ("max $=\% .6 \mathrm{fn} n ", \max$ );

$1 * * * * * * * * * * * * * * * * * * * * * * * * * * * * * * * * * * * * * * * * * * * * * * * * * * * *$

- This routine takes the data set and reduces it to only the injection times wanted.

$* * * * * * * * * * * * * * * * * * * * * * * * * * * * * * * * * * * * * * * * * * * * * * * * * * * * 1$

void

converttowantedinj(rawdata, time, sizetime, first_inj, last_inj)

float **rawdata;

float * time;

int * sizetime;

int first_inj;

int last_inj;

1

int index 1 , index 2 , index 3 ;

*sizetime = last_inj - first_inj +1 ;

if (first_inj > 0 )

for (index $1=0$, index 2 = first_inj; index $2<=$ last_inj; index $1++$, index $2++)$ (

time[index 1$]=$ ime[index 2$]$;

for (index $3=0$; index 3 < NUMDATA; index $3++$ )

rawdata[index 3][index2];

rawdata[index3][index 1] =

了

/****************************************************

- This routine returns the saturated hydraulic conductivity.

$* * * * * * * * * * * * * * * * * * * * * * * * * * * * * * * * * * * * * * * * * * * * * * * * * * * *)$

float

soilprop (thedepth) 
int thedepth;

I

float return_num;

int soiltype;

soiltype = getSoilType(thedepth);

$l^{*}$ the following are normalized between -0.5 and $+0.5 * /$

if $(($ soiltype $=1) \|($ soiltype $=2)$ )

return_num $=-0.5 ; 1^{*} 0.02 * 1$

if (soiltype $=3$ ) return_num $=0.2142857 ; / 0.05 * /$

if (soiltype $=4$ ) return_num $=0.5 ; /^{*} 0.062 * 1$

了

return return_num;

/*****************************************************

- This routine adds soil properties to the data.

- To change the soil properties simply change this routine and the constant NUMSORLPOPS to the number of soilproperties being used.

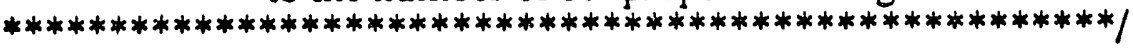

void

getsoilprops (soildata, sizearray, thedepth)

float ** soildata;

int sizearray;

int thedepth;

l

int index 1 ;

static float temp[NUMSOILPROPS];

temp $[0]=$ soilprop(thedepth);

temp $[1]=$ soilprop $($ thedepth +30$)$;

temp $[2]=$ soilprop (thedepth +60$)$;

for (index $1=0$; index $1<$ sizearray; index $1++)$ ( soildata[0][index 1$]=$ temp [0]; soildata[1][index 1$]=$ temp $[1]$;

\} soildata[2][index 1$]=$ temp [2];

\}

f****************************************************

- This routine prints the data out in crenet format.

$* * * * * * * * * * * * * * * * * * * * * * * * * * * * * * * * * * * * * * * * * * * * * * * * * * * * 1$

void

outdata (filename, rawdata, time, soildata, sizearray) 


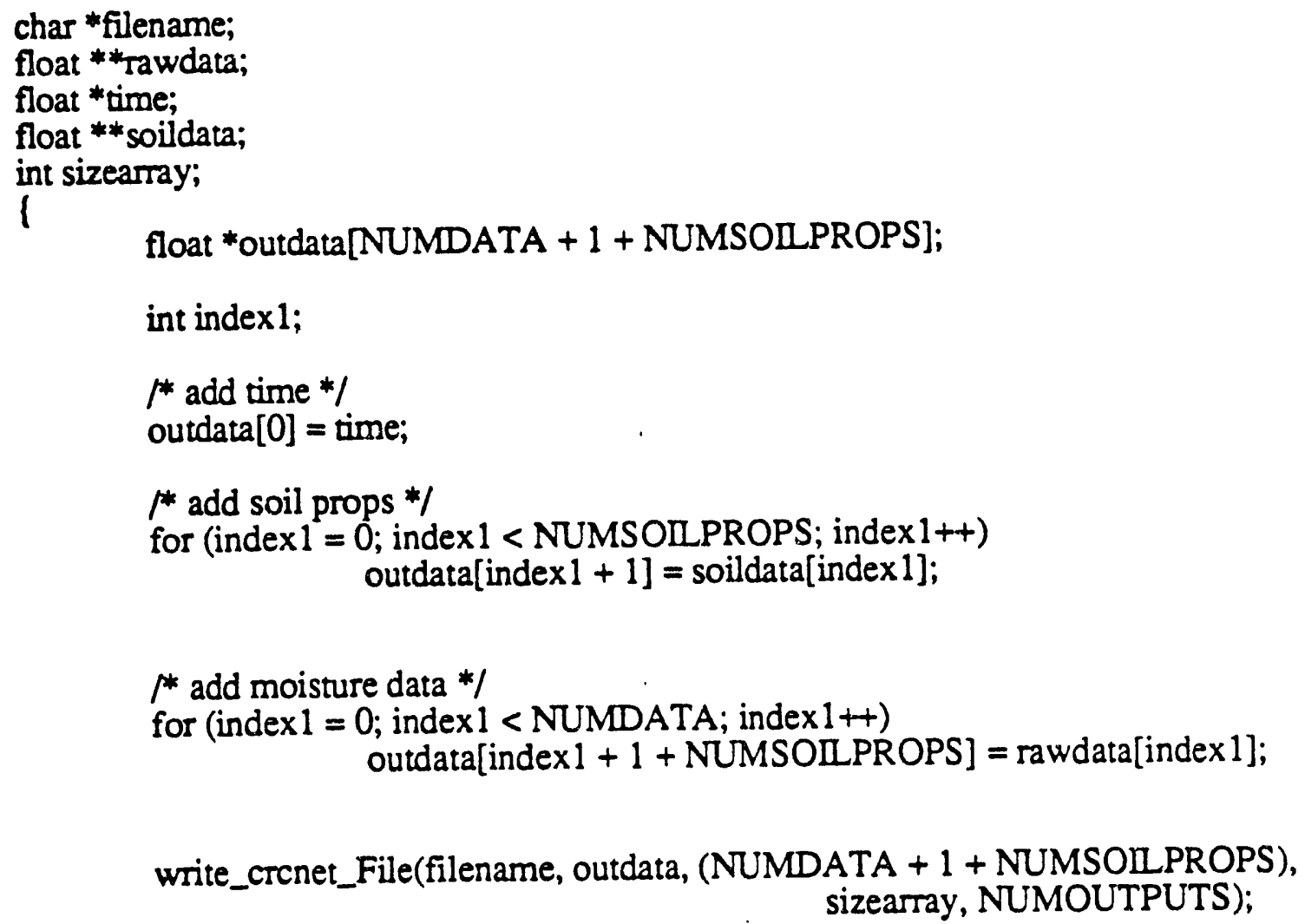

\section{了}

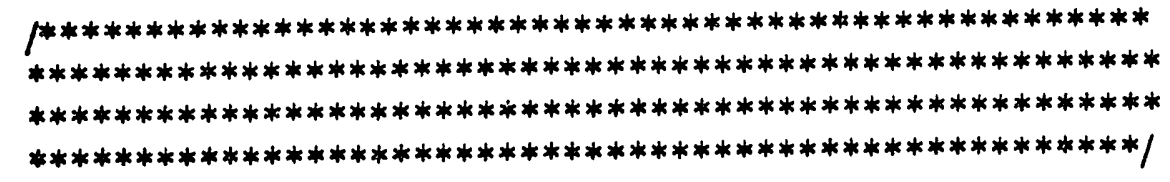

main (argc, argv)

int argc;

char *argv[]; l

float **rawdata, **timedata, **soildata;

static int sizeraw[NUMDATA];

static float time[ARRAYSIZE];

int sizetime;

char *filename;

int thedepth, first_inj, last_inj;

if (argc $!=5)$ (

printf(" ज Usage: outfilename ");

printf("first_inj last_inj depth $(n ")$;

printf(" outfilename $=>$ the output for crenet (rec\#n.input) $\left.n^{\prime \prime}\right)$;

printf(" . first_inj $\Rightarrow>$ the first injection you want data from $h ")$;

printf(" last_inj $\Rightarrow$ the last injection you want data fromln");

printf(" depth $\Rightarrow$ depth of $A \backslash n ")$;

printf(" A B Cn");

printf(" $123 \mathrm{hn");}$ 


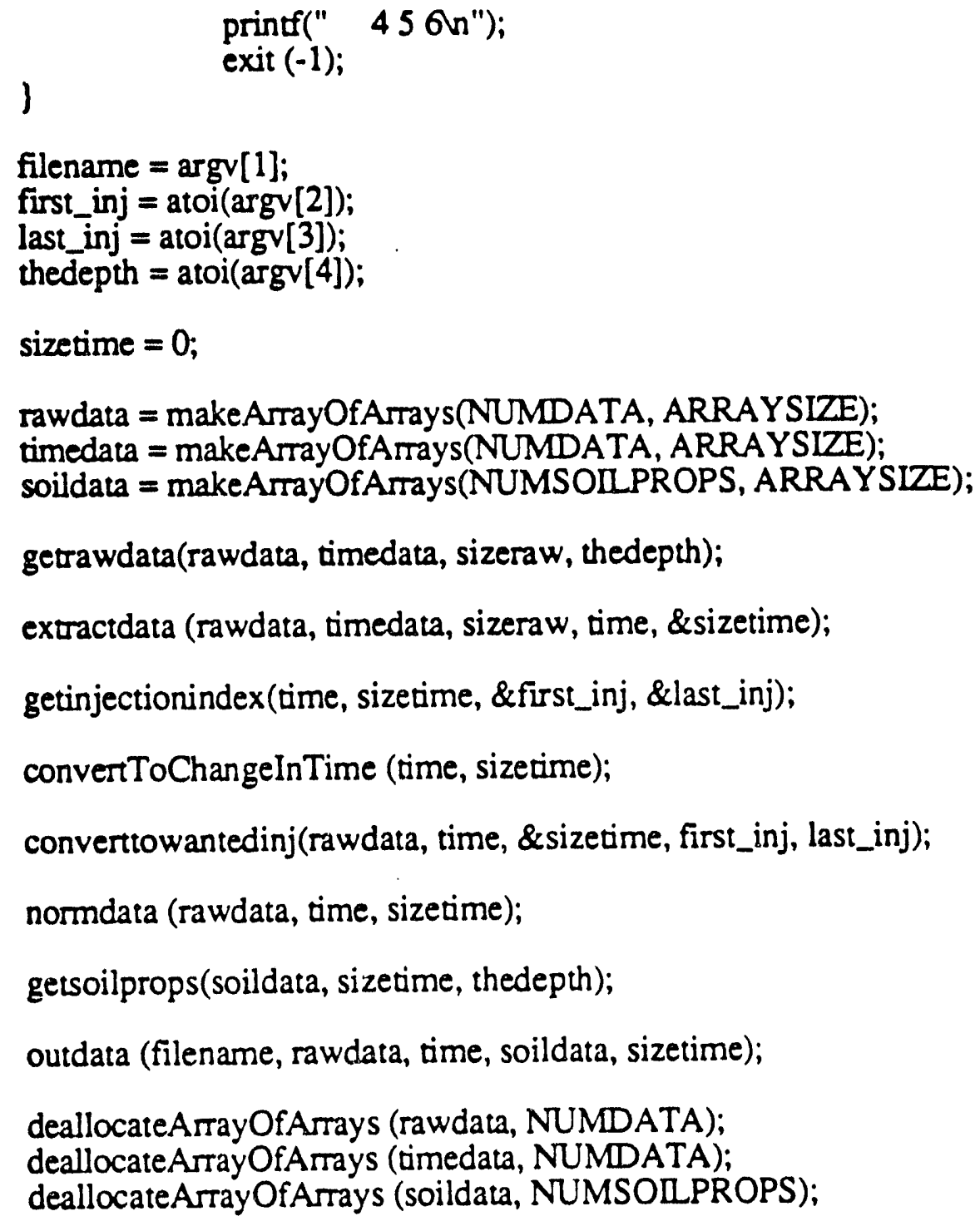




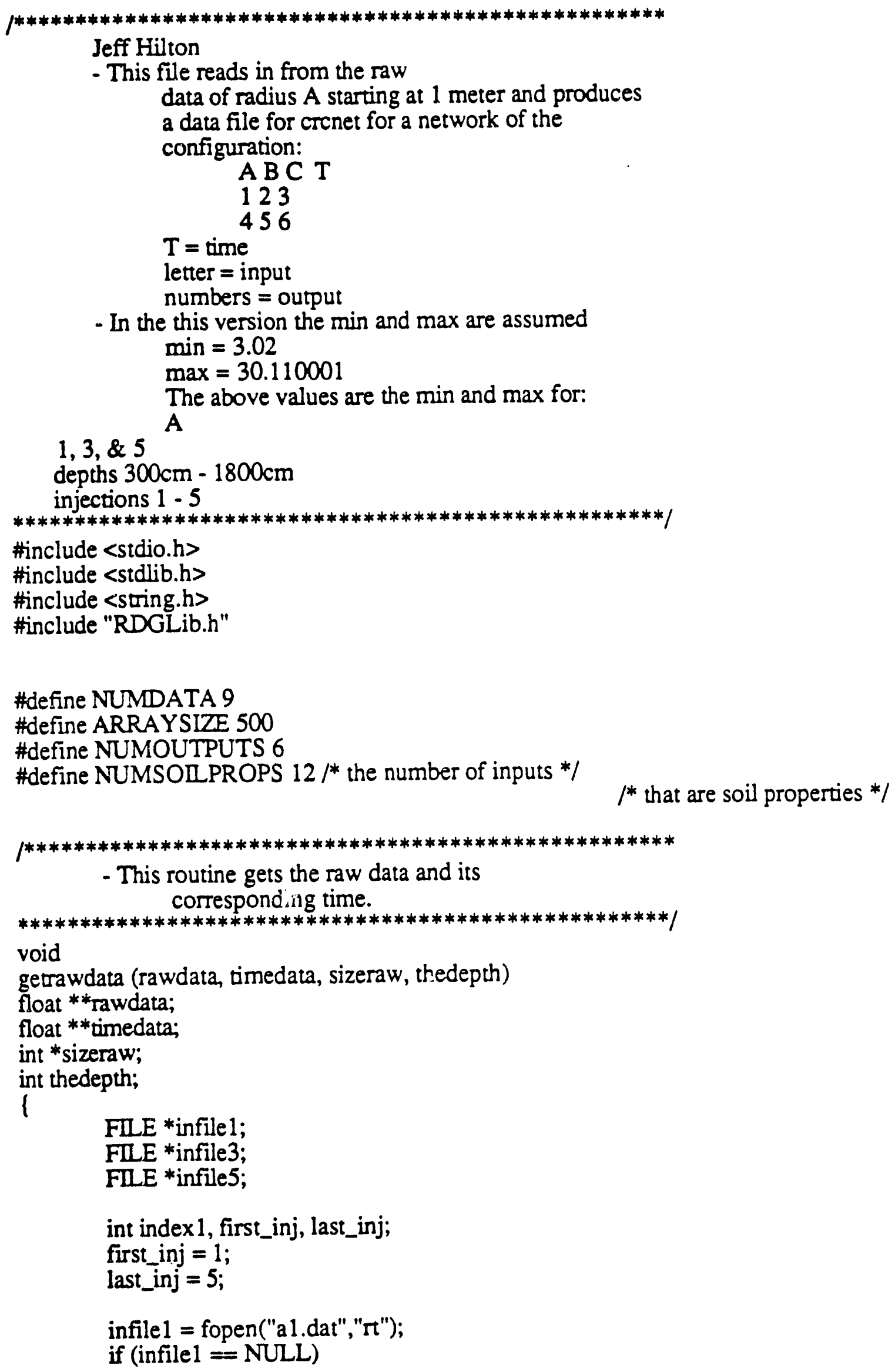

Jeff Hilton

- This file reads in from the raw data of radius $A$ starting at 1 meter and produces a data file for crenet for a network of the configuration:

A B C T

123

456

$\mathrm{T}=$ time

letter $=$ input numbers = output

- In the this version the min and max are assumed $\min =3.02$ $\max =30.110001$

The above values are the min and max for:

$1,3, \& 5$ A

depths $300 \mathrm{~cm}-1800 \mathrm{~cm}$ injections 1 - 5

$* * * * * * * * * * * * * * * * * * * * * * * * * * * * * * * * * * * * * * * * * * * * * * * * * * * * /$

\#include <stdio.h>

\#include <stdlib.h>

\#include <string.h>

\#include "RDGLib.h"

\#define NUMDATA 9

\#define ARRAYSIZE 500

\#define NUMOUTPUTS 6

\#define NUMSORPROPS $12 / *$ the number of inputs */

/*****************************************************

- This routine gets the raw data and its corresponding time.

******************************************************1

void

getrawdata (rawdata, timedata, sizeraw, thedepth)

float **rawdata;

float **timedata;

int *sizeraw;

int thedepth;$$
\text { in }
$$

FIIE *infile 1;

FILE *infile3;

FIIE *infile5;

int index 1, first_inj, last_inj;

first_inj $=1$;

last_inj = 5;

infile1 = fopen("a1.dat"," $r$ ");

if (infilel $=$ NULL) 


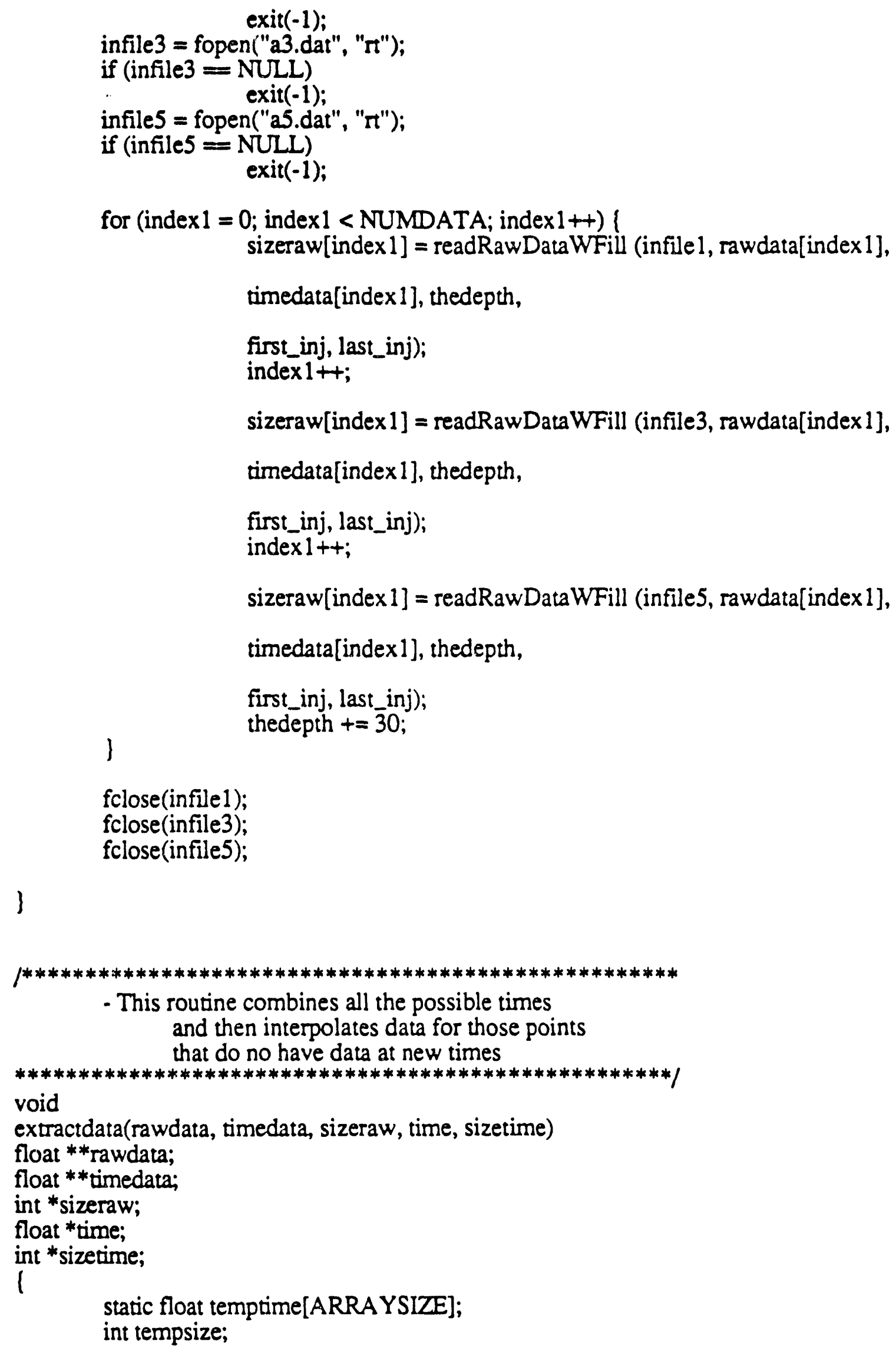

- This routine combines all the possible times and then interpolates data for those points that do no have data at new times

$* * * * * * * * * * * * * * * * * * * * * * * * * * * * * * * * * * * * * * * * * * * * * * * * * * * * /$

void

extractdata(rawdata, timedata, sizeraw, time, sizetime)

float ** rawdata;

float **timedata;

int *sizeraw;

float *time;

int *sizetime;

1

static float temptime[ARRA YSIZE];

int tempsize; 
int index 1 ;

fillTimes (timedata[0], sizeraw[0], timedata[1], sizeraw[1], temptime, \&tempsize);

fillTimes (timedata[2], sizeraw[2], temptime, tempsize, time, sizetime);

for (index 1 = 0 ; index $1<$ NUMDATA; index $1++$ )

fillData(rawdata[index1], timedata[index1], sizeraw[index1], time, *sizetime);

1

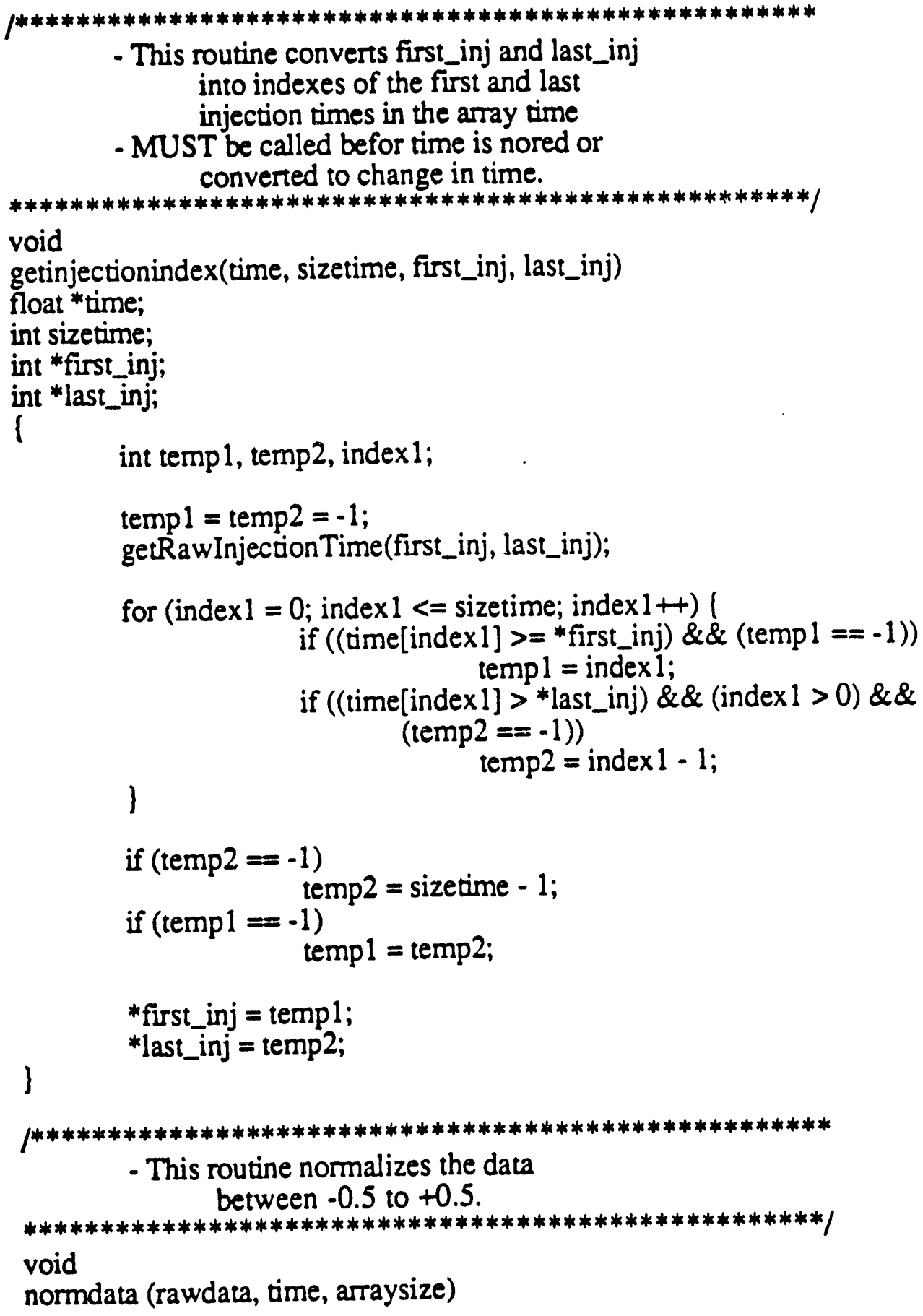

- This routine converts first_inj and last_inj into indexes of the first and last injection times in the array time

- MUST be called befor time is nored or converted to change in time.

$* * * * * * * * * * * * * * * * * * * * * * * * * * * * * * * * * * * * * * * * * * * * * * * * * * * * 1$

void

getinjectionindex(time, sizetime, first_inj, last_inj)

float *time;

int sizetime;

int *first_inj;

int *last_inj;

1

int temp1, temp2, index 1;

templ = temp2 = -1;

getRawInjection Time(first_inj, last_inj);

for (index $1=0$; index $1<=$ sizetime; index $1++)$ (

if $(($ time[index 1$]>=*$ first_inj) \&\& (templ $==-1)$ )

templ = index 1;

if ((time[index 1$]>$ *last_inj) \&\& (index $1>0) \& \&$

(temp2 $==-1$ )) temp2 = index $1-1$

)

if (temp2 $==-1)$

if (templ $=-1)$ temp2 = sizetime - 1; temp1 = temp2;

*first_inj = templ;

*last_inj = temp2;

\}

/*****************************************************

- This routine normalizes the data

between -0.5 to +0.5 .

$* * * * * * * * * * * * * * * * * * * * * * * * * * * * * * * * * * * * * * * * * * * * * * * * * * * *)$

void

normdata (rawdata, time, arraysize) 
float * *rawdata;

float *time;

int arraysize;

1

float min, max;

$\min =3.02$

$\max =30.110001$

normAllData (rawdata, NUMDATA, arraysize, min, max);

$\min =\max =$ time $[0] ;$

getMinMax (time, arraysize, \&min, \&max);

normDataNP5ToPP5(time, arraysize, $\min , \max$ );

printf ("Vnmin = \%.6fn", $\mathrm{min}$ );

)

printf ("max $=\% .6 \mathrm{An} ", \max$ );

\section{p***************************************************}

- This routine takes the data set and reduces it to only the injection times wanted.

****************************************************1

void

convertowantedinj(rawdata, time, sizetime, first_inj, last_inj)

float **rawdata;

float * time;

int *sizetime;

int first_inj;

int last_inj;

l

int index 1 , index 2 , index 3 ;

*sizetime = last_inj - first_inj + 1;

if (first_inj $>0$ )

for (index $1=0$, index $2=$ first_inj; index $2<=$ last_inj;

index $1++$, index $2++)$ (

time[index1] = time[index2];

for (index $3=0$; index $3<$ NUMDATA; index $3+$ )

rawdata[index 3][index 2];

rawdata[index3][index1] =

了

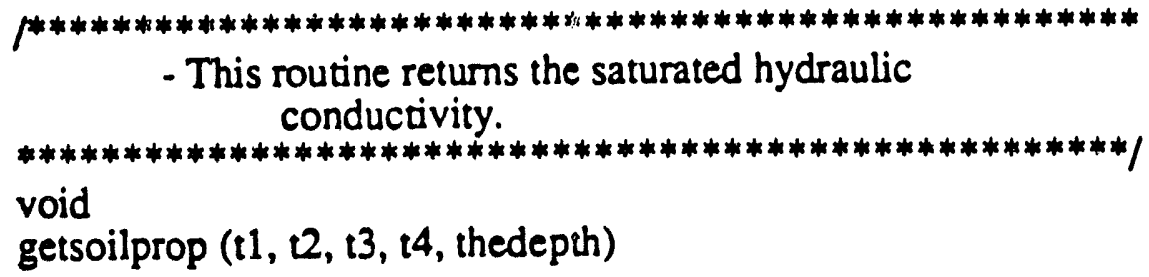


float *t1;

float *2;

float * 3 ;

float * 4 ;

int thedepth;

int soiltype;

soiltype $=$ getSoilType(thedepth $)$;

* the following are normalized between -0.5 and $+0.5 * /$

if (soiltype $=1$ ) (

$$
\begin{aligned}
& * t 1=0.5 ; \\
& * t 2=-0.5 ; \\
& * t 3=-0.5 ; \\
& * t 4=-0.5 ;
\end{aligned}
$$

)

if (soiltype $==2$ ) (

$*_{t 1}=-0.5$;

$* 2=0.5$;

$*$ t3 = -0.5;

$* t 4=-0.5$;

)

$$
\text { if (soiltype = } \begin{aligned}
3) & 1 \\
* t 1 & =-0.5 \\
* t 2 & =-0.5 \\
* t 3 & =0.5 \\
* t 4 & =-0.5
\end{aligned}
$$

\}

if (soiltype $==4$ )

$* t 1=-0.5$

$* 12=-0.5$

$*$ t3 $=-0.5$

$*$ t4 = 0.5;

\}

\}

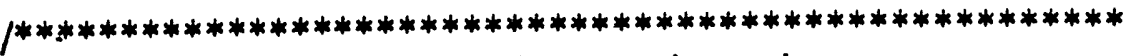

- This routine adds soil properties to the data.

- To change the soil properties simply change this routine and the constant NUMSOILPROPS to the number of soilproperties being used.

$* * * * * * * * * * * * * * * * * * * * * * * * * * * * * * * * * * * * * * * * * * * * * * * * * * * *)$

void

getsoilprops (soildata, sizearray, thedepth) float ** soildata;

int sizearray;

int thedepth;

int index 1 , index 2 ;

static float temp[NUMSOILPROPS]; 
getsoilprop (\&temp[0], \&temp[1], \&temp[2], \&temp [3], thedepth);

getsoilprop (\&temp [4], \&temp[5], \&temp[6], \&temp[7], thedepth + 30);

getsoilprop (\&temp[8], \&temp[9], \&temp[10], \&temp[11], thedepth +60);

for (index $1=0$; index $1<$ NUMSOILPROPS; index $1++$ )

for (index $2=0$; index $2<$ sizearray; index $2++$ )

soildata[index 1$][$ index 2$]=$ temp[index 1$]$;

1 /****************************************************

- This routine prints the data out in crenet format.

****************************************************|

void

outdata (filename, rawdata, time, soildata, sizearray)

char *filename;

float **rawdata;

float *time;

float ** soildata;

int sizearray;

I

float *outdata[NUMDATA + 1 + NUMSOILPROPS];

int index 1;

$1 *$ add time */

outdata $[0]=$ time;

$1 *$ add soil props */

for (index $1=0$; index $1<$ NUMSOILPROPS; index $1++$ )

outdata[index $1+1]$ = soildata[index 1$]$;

$1 *$ add moisture data */

for (index $1=0$; index $1<$ NUMDATA; index $1+$ )

outdata[index $1+1+$ NUMSOILPROPS] = rawdata[index 1];

write_crenet_File(filename, outdata, (NUMDATA + 1 + NUMSOIPPROPS), sizearray, NUMOUTPUTS);

\}

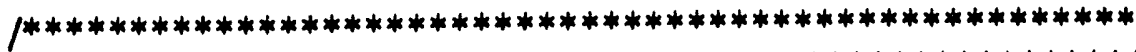

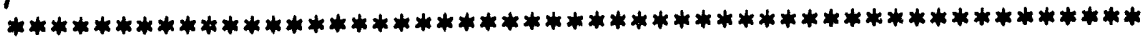

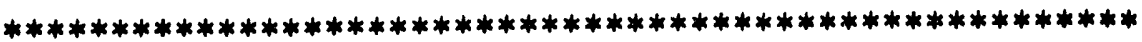

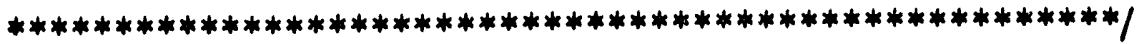

main (argc, argv)

int argc;

char *argv口;

l

float **rawdata, **timedata, **soildata; 
static int sizeraw[NUMDATA];

static float time[ARRA YSIZE];

int sizetime;

char *filename;

int thedepth, first_inj, last_inj;

if $(\operatorname{argc} !=5)$ ( printf(" In Usage: outfilename "); printf("first_inj last_inj depthln"); prinff(" outfilename $=>$ the output for crenet (rec\#n.input) $) n "$ "); printf(" first_inj $\Rightarrow>$ the first injection you want data from $n "$ "); printf (" last_inj $\Rightarrow>$ the last injection you want data fromb"); printf(" depth $\Rightarrow$ depth of $A$ ln"); printf(" A B Cn");

printf(" 123 n"); printf(" 45 on");

1 exit $(-1)$;

filename $=\operatorname{argv}[1]$

first_inj = atoi (argv[2]);

last_inj $=$ atoi $(\operatorname{argv}[3])$;

thedepth = atoi (argv[4]);

sizetime $=0$;

rawdata = makeArrayOfArtays (NUMDATA, ARRAYSIZE);

timedata = makeArrayOfArrays(NUMDATA, ARRA YSIZE);

soildata = makeArayOfArrays(NUMSOILPROPS, ARRAYSIZE);

getrawdata(rawdata, timedata, sizeraw, thedepth);

extractdata (rawdata, timedata, sizeraw, time, \&sizetime);

getinjectionindex(time, sizetime, \&first_inj, \&last_inj);

convertToChangeInTime (time, sizetime);

converttowantedinj(rawdata, time, \&sizetime, first_inj, last_inj);

normdata (rawdata, time, sizetime);

getsoilprops(soildata, sizetime, thedepth);

outdata (filename, rawdata, time, soildata, sizetime);

deallocateArrayOfArrays (rawdata, NUMDATA);

deallocateArrayOfArrays (timedata, NUMDATA);

deallocateArrayOfArrays (soildata, NUMSOILPROPS); 
// this program only works for a resolution of 0.05

// this produces 7721 times sieps

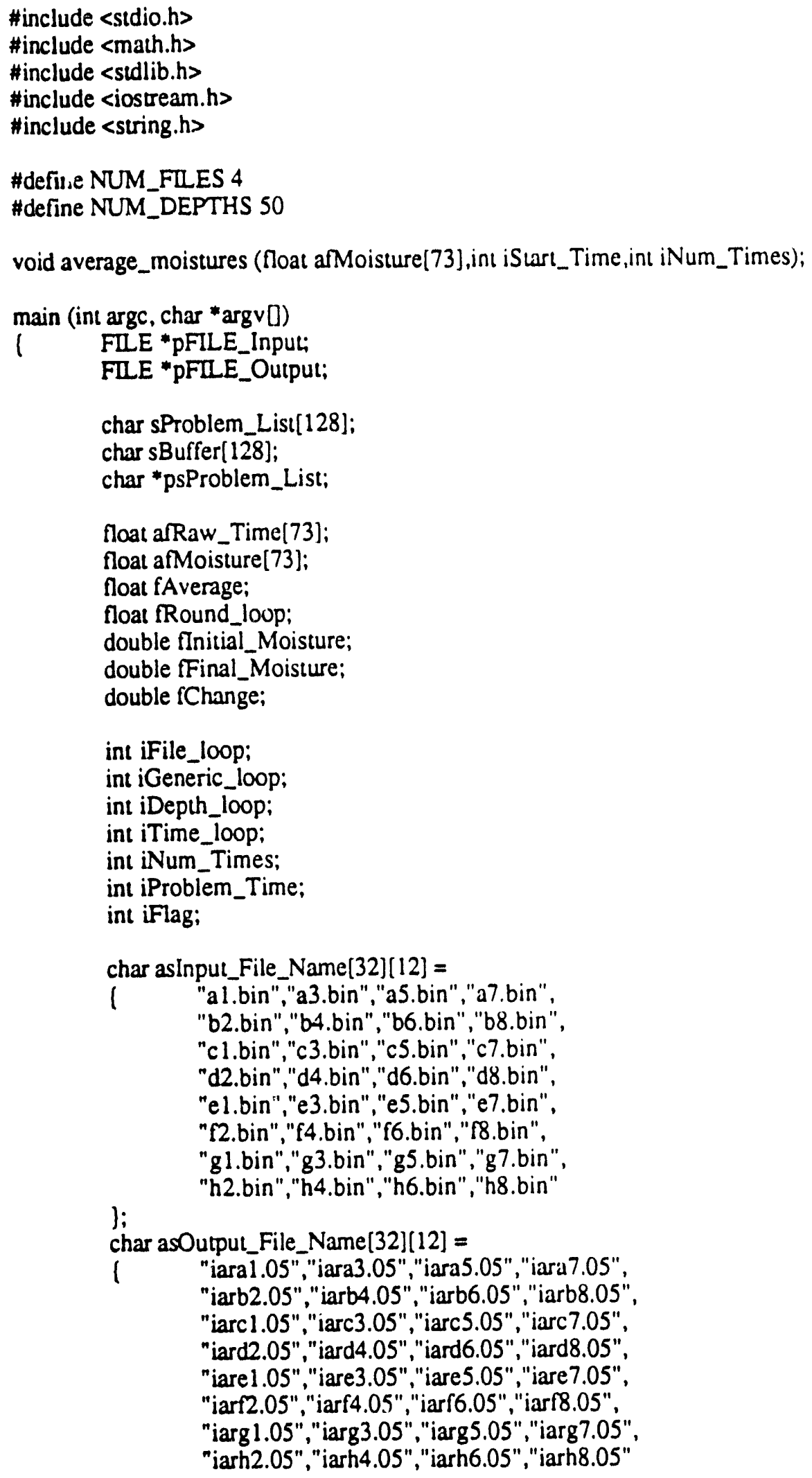


; ;

for (iFile_loop $=0$;iFile_loop $<$ NUM_FILES;iFile_loop ++ )

( cout $\ll<$ "Processing file: " $\ll$ asInput_File_Name[iFile_loop] $\ll$ h'; pFILE_Input $=$ fopen(asInput_File_Name[iFile_loop],"rb");

pFILE_Output $=$ fopen(asOutput_File_Name(iFile_loop],"w");

fread (afRaw_Time, sizeof (afRaw_Time),1,pFILE_Input); // read the time stumps

for(iTime_loop $=0$;iTime_loop $<73 ;$ iTime_loop++)

1 for (fRound_loop $=0$; IRound_loop $<386$; IRound_loop $+=0.05$ )

1 if (fabs(Round_loop - arRaw_Time[iTime_loop]) $<=0.025$ )

1 afRaw_Time[iTime_loop] = Round_loop;

)

RRound_loop = 386;

) // round the time stamps

sprintf (sProblem_List,"");

for (iTime_loop $=0$;iTime_loop $<$ 72;iTime_loop ++ )

if $(($ arRaw_Time [iTime_loop] $!=0) \& \&$

(arRaw_Time [iTime_loop] $==$ atRaw_Time[iTime_loop+1]))

( sprints (sBuffer,"\%d ",iTime_loop); strcal (sProblem_List,sBuffer);

for (iGeneric_loop $=$ iTime_loop;iGeneric_loop < 72;iGeneric_loop++)

arRaw_Time[iGeneric_loop] $=a \sqrt{R} a w_{-}$Time [iGeneric_loop +1$]$;

arRaw_Time $[7 \overline{2}]=0.0$;

)

// remove any overlapping times

iGeneric_loop $=0$;

for (fRound_loop $=0$; Round_loop $<386$; Round_loop $+=0.05$ )

fprintf (pFILE_Output," \%.2f ",Round_loop);

fprintf (pFILE_Output," "ר");

for (iDepth_loop $=0$;iDcplh_loop < NUM_DEPTHS;iDcpth_loop++)

( cout $\ll<"$ depth.........." $<$ iDepth_loop $\ll<V^{\prime}$;

fread (afMoisture,size of (alMoisture), 1,pFILE_Input);

$/ /$ read current depth

for (iTime_loop =0:iTime_loop $<$ 72;iTime_loop++)

[ if (arMoistu-e[iTime_loop] $==100$ )

73;iGeneric_loop++)

if (iTime_loop $==0$ )

1 for (iGeneric_loop $=$ iTime_loop $+1 ;$ iGeneric_loop $<$

afMoisture[iGeneric_loop];

73;iGeneric_loop++)

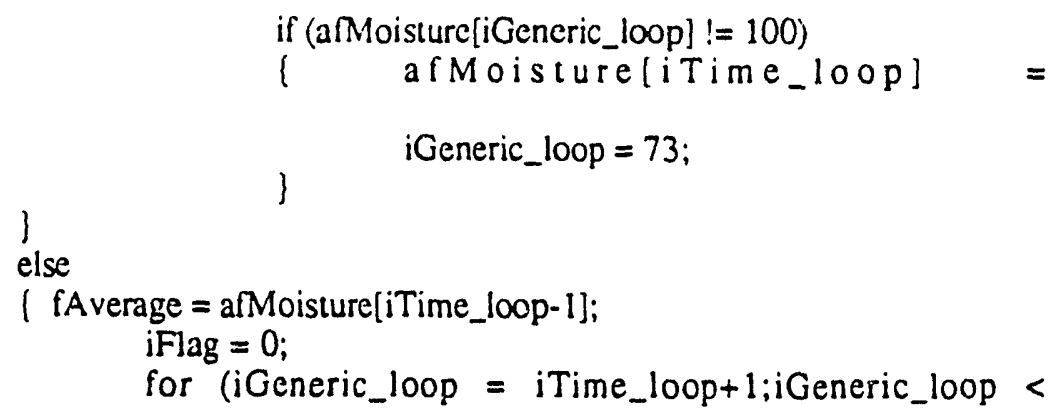

if (afMoisture[iGeneric_loop] !=100.0)

( fAverage $+=\mathrm{a}$ MMoisture[iGeneric_loop];

iGcneric_loop =73; 
72;iGeneric_loop++)

1])*20);

) // cycle through the depths averaging whereever a non-repon is found

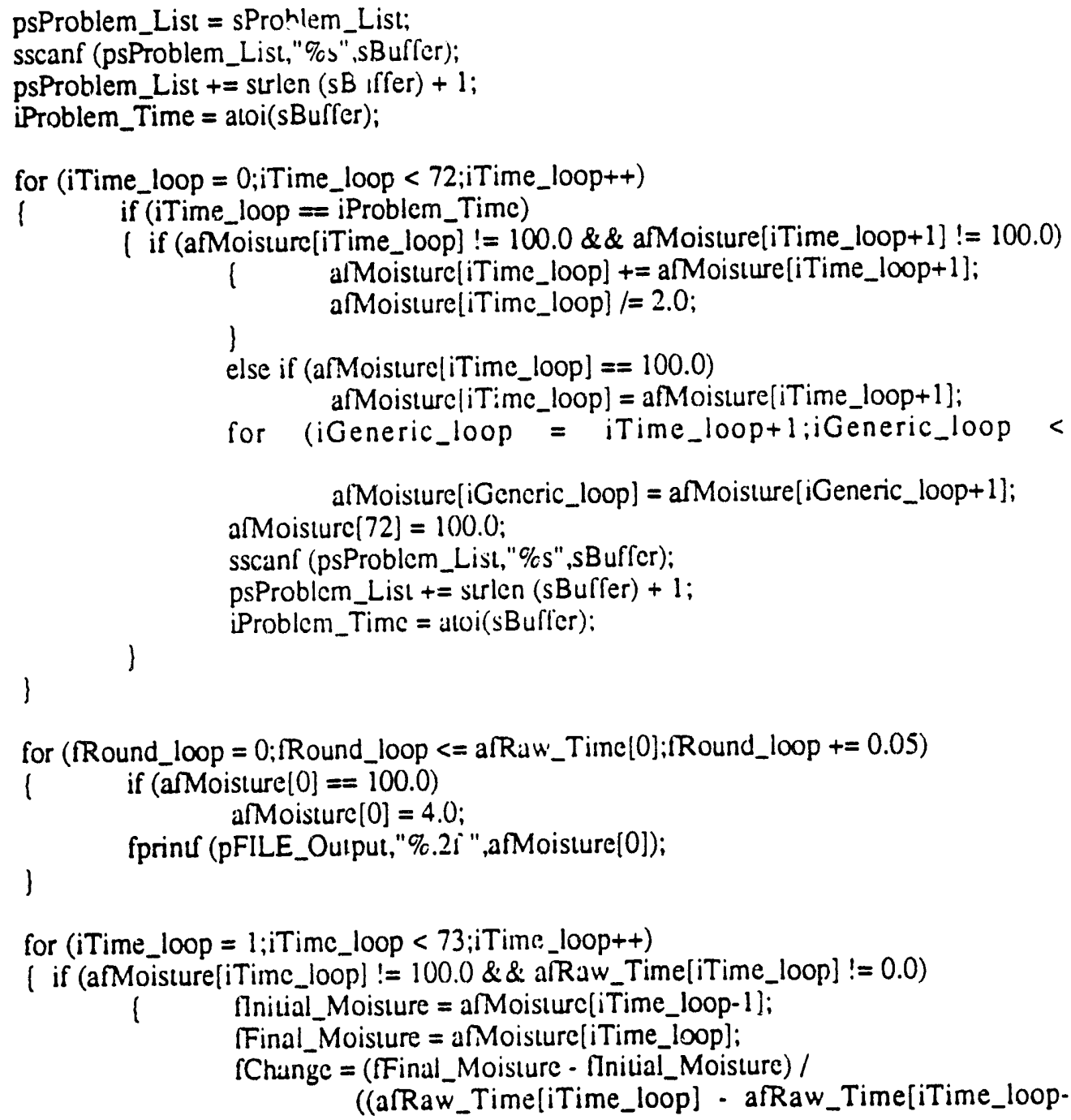

for (Round_loop $=$ arRaw_Time[iTime_loop-1] + 0.05;

Round_loop < atRaw_Time[iTime_loop];

(Round_loop $+=0.05$ )

( Initial_Moisture $+=$ \{Change;

fprintf (pFILE_Output,"\%.2f ",Initial_Moisture);

1

I

fprinuf (pFILE_Outpul,"\%.2f ",arMoisture[iTime_loop]);

else

$1 \quad$ Round_loop $=$ iTime_loop -1 ;

iTime_loop = 73; 


$$
\begin{aligned}
& \text { \} } \\
& \text { iTime_loop = [Round_loop; } \\
& \text { for (RRound_loop = arRaw_Time [iTime_loop] + 0.05; }
\end{aligned}
$$$$
\text { fRound_loop < 386; }
$$$$
\text { fRound_loop }+=0.05 \text { ) }
$$$$
\text { fprintf (pFILE_Output,"\%.2f ",arMoisture[iTime_loop]); }
$$

fprintf (pFILE_Output, "ר");

)

fclose (pFILE_Input);

fclose (pFILE_Output); 


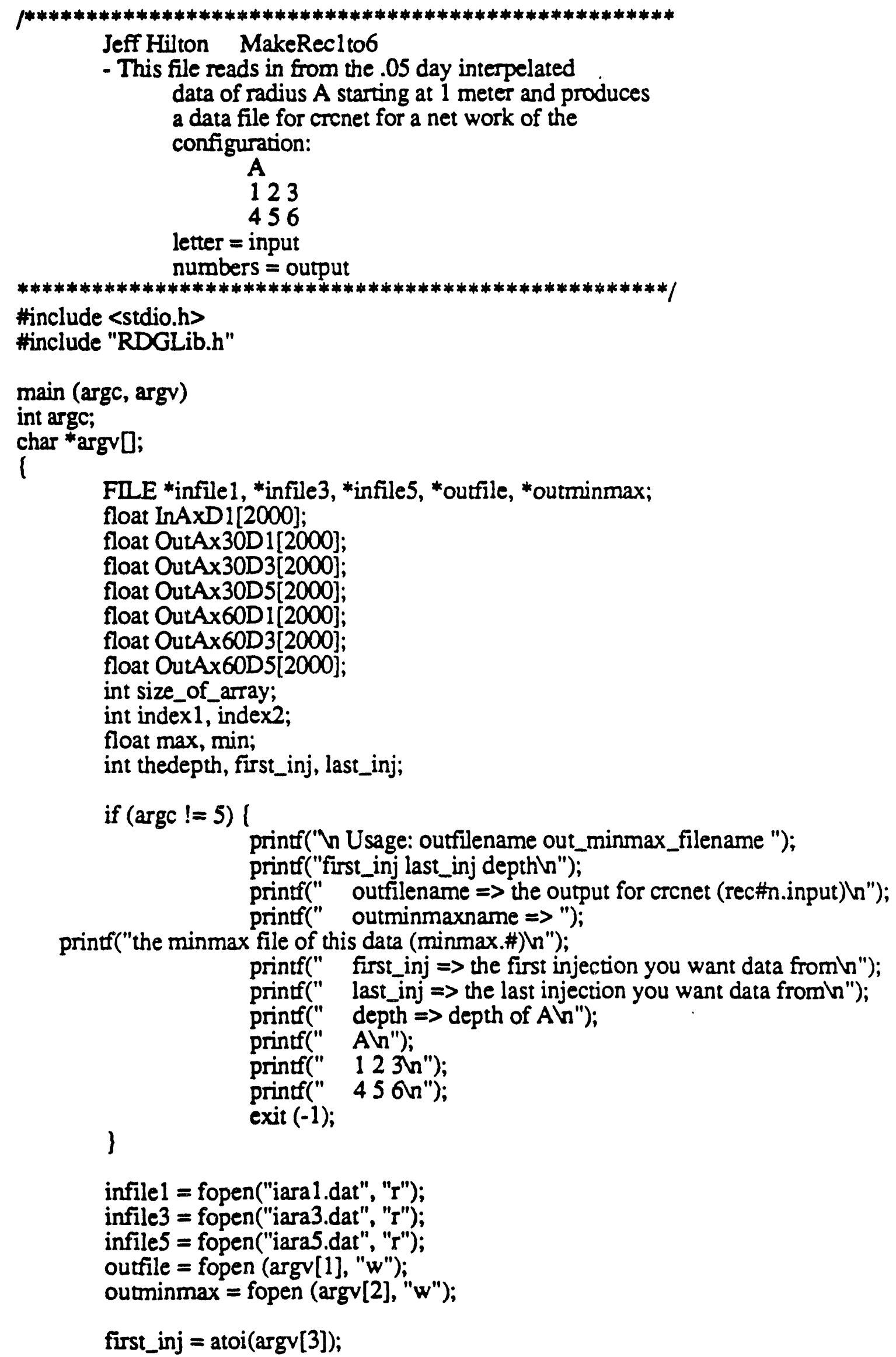


last_inj = atoi(argv[4]);

thedepth = atoi(argv[5]);

size_of_array $=$ readADepth(infile $1, \operatorname{InAxD} 1$, thedepth,

first_inj,

last_inj);

readADepth (infile1, OutAx30D1, thedepth + 30, first_inj, last_inj);

readADepth (infile1, OutAx60D1, thedepth +60 , first_inj, last_inj);

readADepth (infile3, OutAx30D3, thedepth +30 , first_inj, last_inj);

readADepth (infile3, OutAx60D3, thedepth + 60, first_inj, last_inj);

readADepth (infile5, OutAx30D5, thedepth + 30, first_inj, last_inj);

readADepth (infile5, OutAx60D5, thedepth +60 , first_inj, last_inj);

$\min =\operatorname{In} A \times D 1[0]$

$\max =\operatorname{InAxD1}[0]$

getMinMax (InAxD1, size_of_array, \&min, \&max);

getMinMax(OutAx30D1, size_of_array, \&min, \&max);

getMinMax(OutAx30D3, size_of_array, \&min, \&max);

getMinMax(OutAx30D5, size_of_array, \&min, \&max);

getMinMax(OutAx60D1, size_of_array, \&min, \&max);

getMinMax(OutAx60D3, size_of_array, \&min, \&max);

getMinMax(OutAx60D5, size_of_array, \&min, \&max);

fprintf(outminmax, "\%.6f \%.6fn", min, max);

normData0To1(InAxD1, size_of_array, $\min , \max$ );

normData0Tol(OutAx30D1, size_of_array, min, $\max$ );

normData0Tol(OutAx30D3, size_of_array, min, max);

normData0Tol(OutAx30D5, size_of_array, min, $\max$ );

normData0Tol(OutAx60D1, size_of_array, $\min , \max$ );

normData0Tol(OutAx60D3, size_of_array, min, max);

normData0Tol(OutAx60D5, size_of_array, min, max);

fprintf(outfile, "\%d 1 6n", size_of_array);

for (index $1=0$; index $1<$ size_of_array; index $1++$ )

fprintf(outfile, "\%.6f \%.6f \%.6f \%.6f \%.6f \%.6f \%.6fn",

InAxD1 [index 1],

OutAx30D1[index1],

OutAx30D3[index1],

OutAx30D5[index1],

OutAx60D1 [index1],

OutAx60D3[index1],

OutAx60D5[index1]);

fclose(infile1);

fclose(infile3);

fclose(infile5);

fclose(outfile);

fclose(outminmax); 


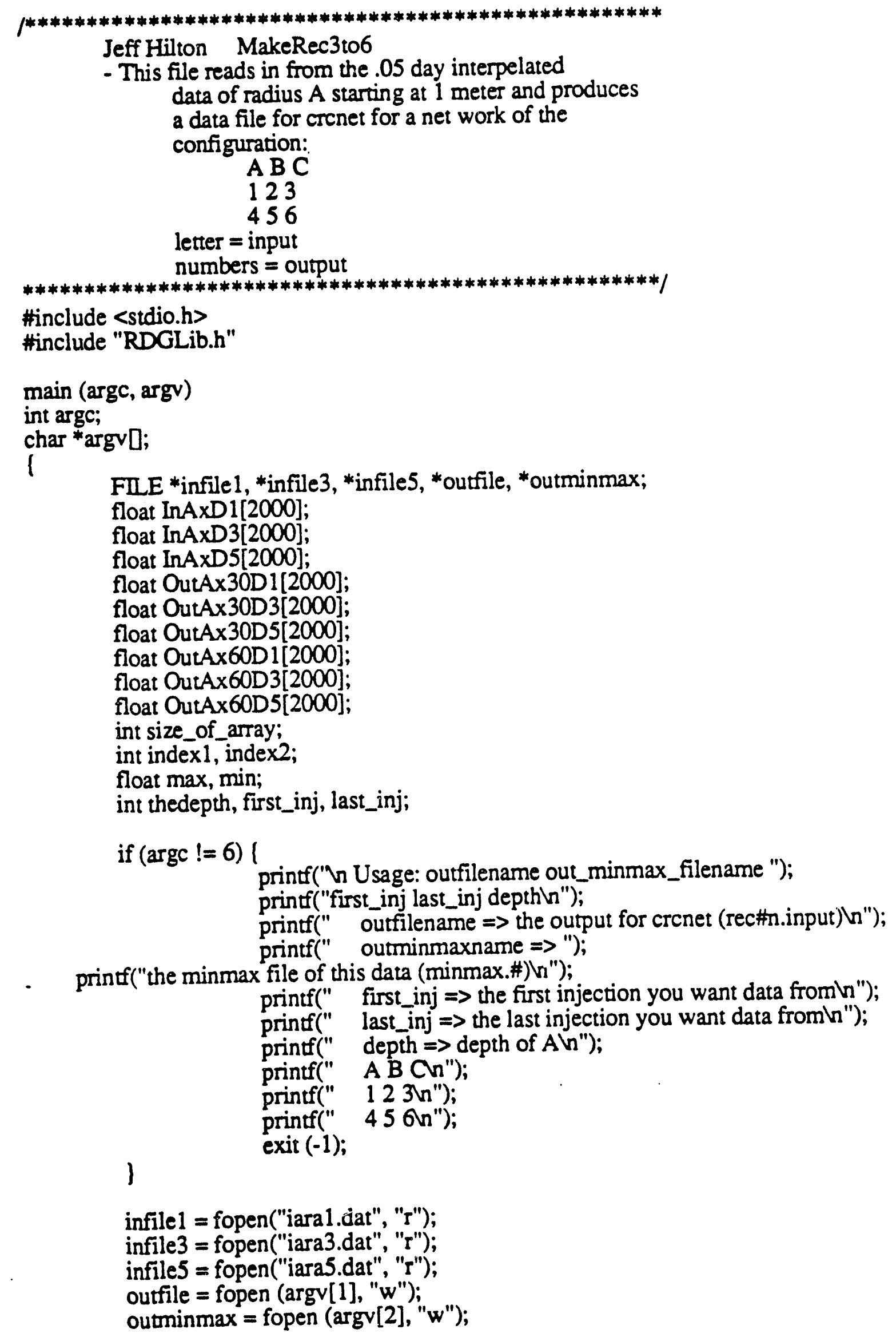


first_inj = atoi(argv[3]);

last_inj = atoi(argv[4]);

thedepth $=$ atoi $(\operatorname{argv}[5])$;

size_of_array $=$ readADepth(infile $1, \operatorname{In} A x D 1$, thedepth,

first_inj,

last_inj);

readADepth (infile1, OutAx30D1, thedepth + 30, first_inj, last_inj); readADepth (infile1, OutAx60D1, thedepth +60 , first_inj, last_inj); readADepth (infile3, InAxD3, thedepth, first_inj, last_inj); readADepth (infile3, OutAx30D3, thedepth + 30, first_inj, last_inj); readADepth (infile3, OutAx60D3, thedepth +60 , first_inj, last_inj); readADepth (infile5, InAxD5, thedepth, first_inj, last_inj);

readADepth (infile5, OutAx30D5, thedepth + 30, first_inj, last_inj); readADepth (infile5, OutAx60D5, thedepth +60, first_inj, last_inj);

$\min =\operatorname{InAxD1}[0]$

$\max =\operatorname{InAxD1}[0]$

getMinMax(InAxD1, size_of_array, \&min, \&max);

getMinMax(InAxD3, size_of_array, \&min, \&max);

getMinMax ( $\Omega A x D 5$, size_of_array, \&min, \&max);

getMinMax(OutAx30D1, size_of_array, \&min, \&max);

getMinMax(OutAx30D3, size_of_array, \&min, \&max);

getMinMax(OutAx30D5, size_of_array, \&min, \&max);

getMinMax(OutAx60D1, size_of_array, \&min, \&max);

getMinMax(OutAx60D3, size_of_array, \&min, \&max);

getMinMax(OutAx60D5, size_of_array, \&min, \&max);

fprintf(outminmax, "\%.6f \%.6fn", min, max);

normData0Tol (InAxD1, size_of_array, min, $\max$ );

normData0Tol(InAxD5, size_of_array, $\min , \max$ );

normData0To1(InAxD3, size_of_array, $\min , \max$ );

normData0Tol(OutAx30D1, size_of_array, $\min , \max$ );

normData0To1(OutAx30D3, size_of_array, min, max);

normDataOTol(OutAx30D5, size_of_array, min, max);

normData0To1(OutAx60D1, size_of_array, min, max);

normData0To1(OutAx60D3, size_of_array, min, max);

normData0To1(OutAx60D5, size_of_array, min, $\max$ );

fprintf(outfile, "\%d 3 $\mathrm{n}$ ", size_of_array);

for (index $1=0$; index $1<$ size_of_array; index $1++$ )

\%.6nn",

fprintf(outfile, "\%.6f \%.6f \%.6f \%.6f \%.6f \%.6f \%.6f \%.6f

\author{
In $A \times D 1$ [index 1$]$, \\ In $A x D 3[$ index 1], \\ InAxD5[index 1], \\ OutAx30D1[index1], \\ OutAx30D3[index1], \\ OutAx30D5[index1], \\ OutAx60D1[index1], \\ OutAx60D3[index1],
}




\section{MakeRec3to6.c}

OutAx60D5[index1]);

fclose(infile1);

fclose(infile 3);

fclose(infile5);

fclose(outfile);

fclose(outminmax); 


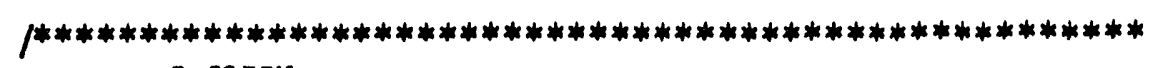

Jeff Hilton

- This file reads in from the .05 day interpelated

data of radius $A$ starting at 1 meter and produces

a data file for crenet for a net work of the

configuration:

A B C

123

456

letter $=$ input

numbers = output

- In the $B$ version the min and max are assumed

$\min =3.02$

$\max =30.110001$

This is the min and max for

A

$1,3, \& 5$

depths $300 \mathrm{~cm}-1800 \mathrm{~cm}$ injections 1.5

*****************************************************1

\#include <stdio.h>

\#include "RDGLib.h"

main (argc, argv)

int argc;

char *argv0;

l

FILE *infile1, *infile3, *infile5, *outfile;

float InAxD1[2000];

float $\operatorname{In} A x D 3[2000]$;

float InAxD5[2000];

float OutAx30D1[2000];

float OutAx30D3[2000];

float OutAx30D5[2000];

float OutAx60D1[2000];

float OutAx60D3[2000];

float OutAx60D5[2000];

int size_of_array;

int index 1 , index 2 ;

float $\max , \mathrm{min}$;

int thedepth, first_inj, last_inj;

if $(\operatorname{argc} !=5)$ !

printf("In Usage: outfilename ");

printf("first_inj last_inj depth $\backslash$ ");

printf(" outfilename => the output for crenet (rec\#n.input) $(n ")$;

printf(" first_inj $\Rightarrow>$ the first injection you want data from $\left.n^{\prime \prime}\right)$;

printf(" last_inj $\Rightarrow$ the last injection you want data from $\backslash "$ ");

printf(" depth $\Rightarrow$ depth of $A$ ln");

printf(" A B Cn");

printf(" $123 \mathrm{hn}$ ");

printf(" 45 on");

)

exit $(-1)$; 


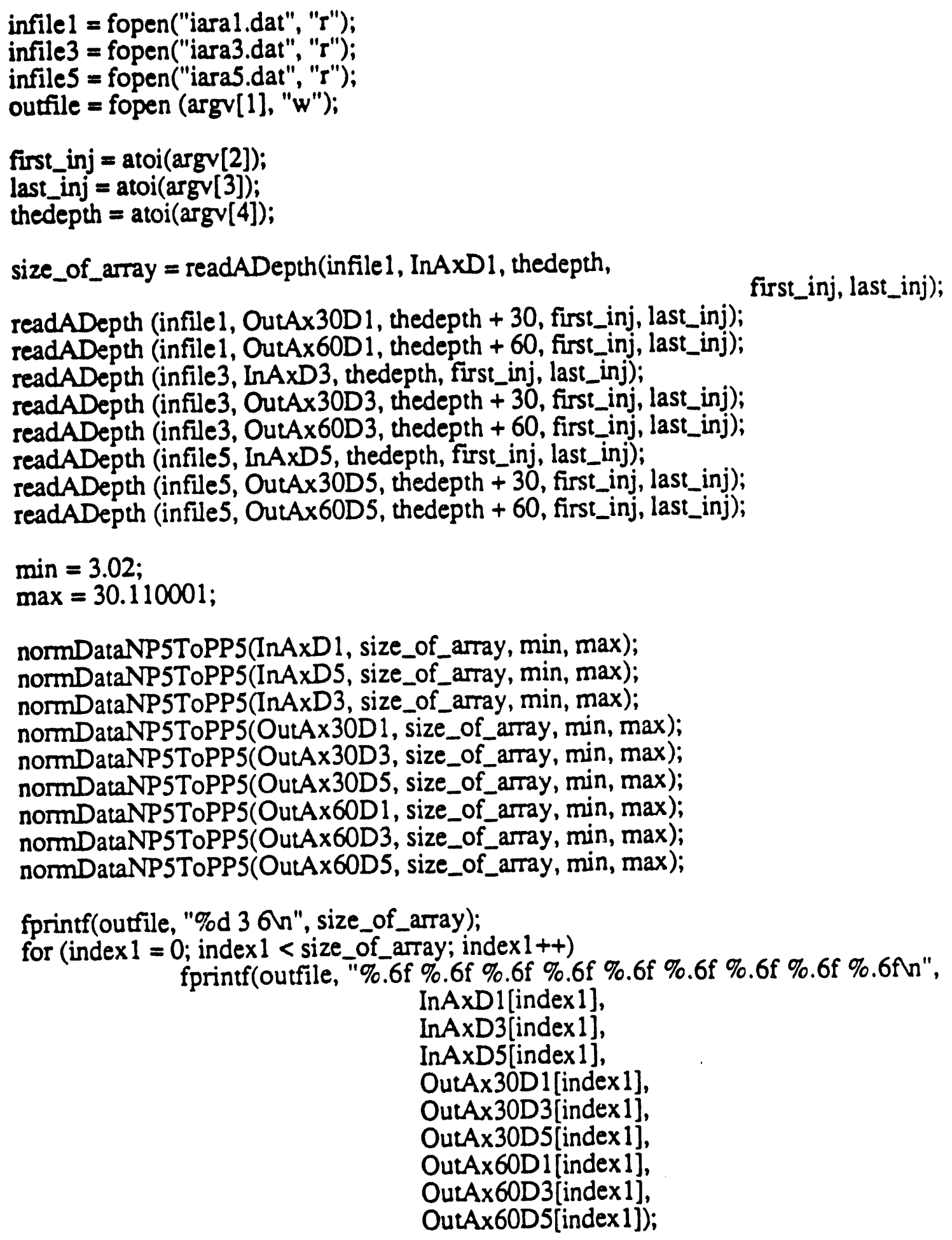

fclose(infile1);

fclose(infile3);

fclose(infile5);

fclose(outrile); 


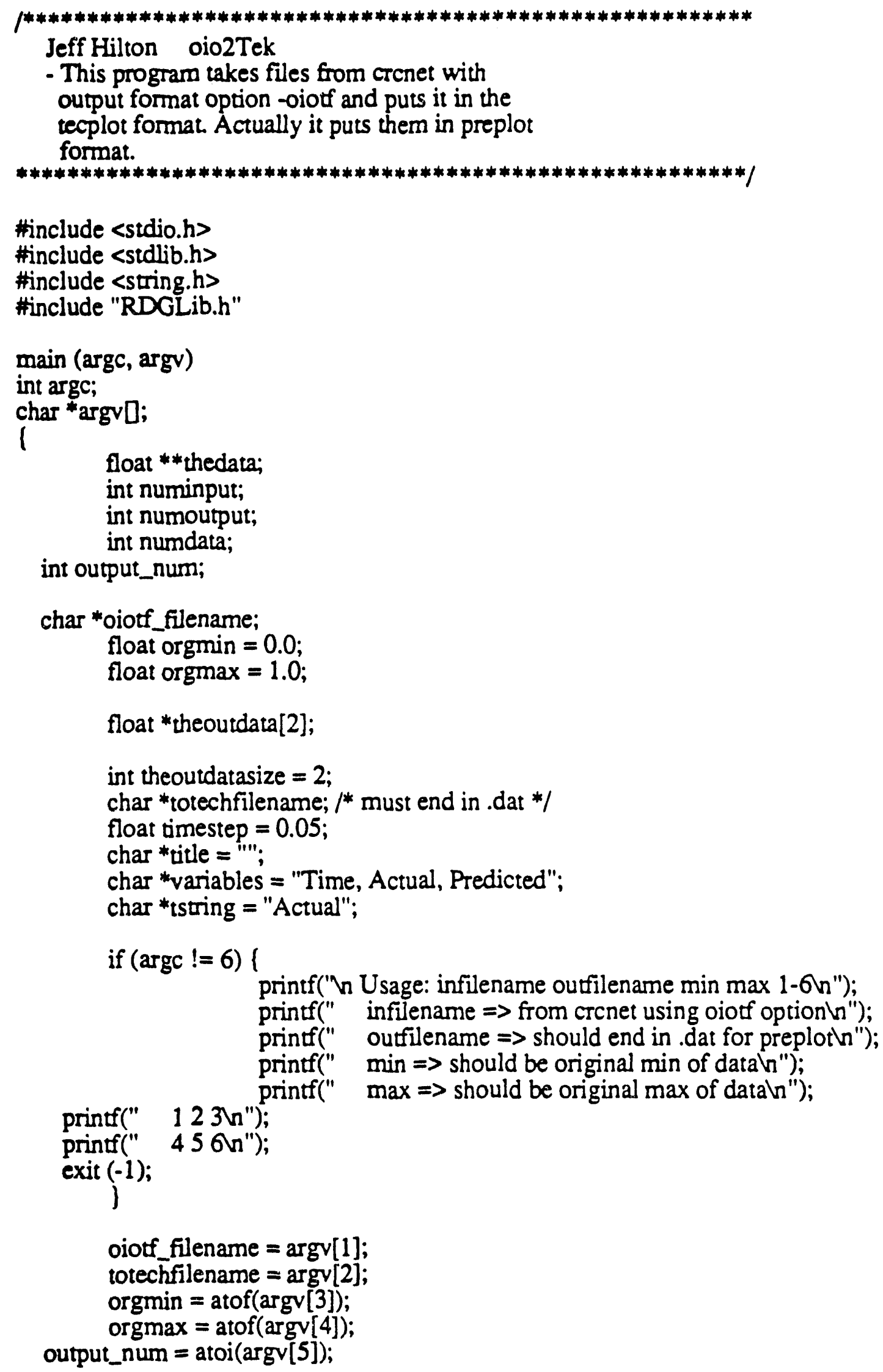

Jeff Hilton oio2Tek

- This program takes files from crenet with

output format option -oiot and puts it in the

tecplot format. Actually it puts them in preplot

format.

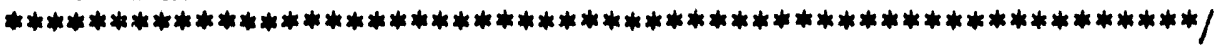


read_oiotf_File (oiotf_filename, \&thedata, \&numinput, \&numoutput,

\&numdata, orgmin, orgmax);

$/ *$ get the first of the training and the first of the output */

theoutdata[0] = thedata[numinput + numoutput + output_num - 1];

theoutdata[1] = thedata[numinput + output_num - 1];

write_TechPlot_File (totechfilename, theoutdata, theoutdatasize,

title, variables,

numdata, timestep, NULL,

tstring);

deallocate_oiotf_Data (thedata, numinput, numoutput);

1 


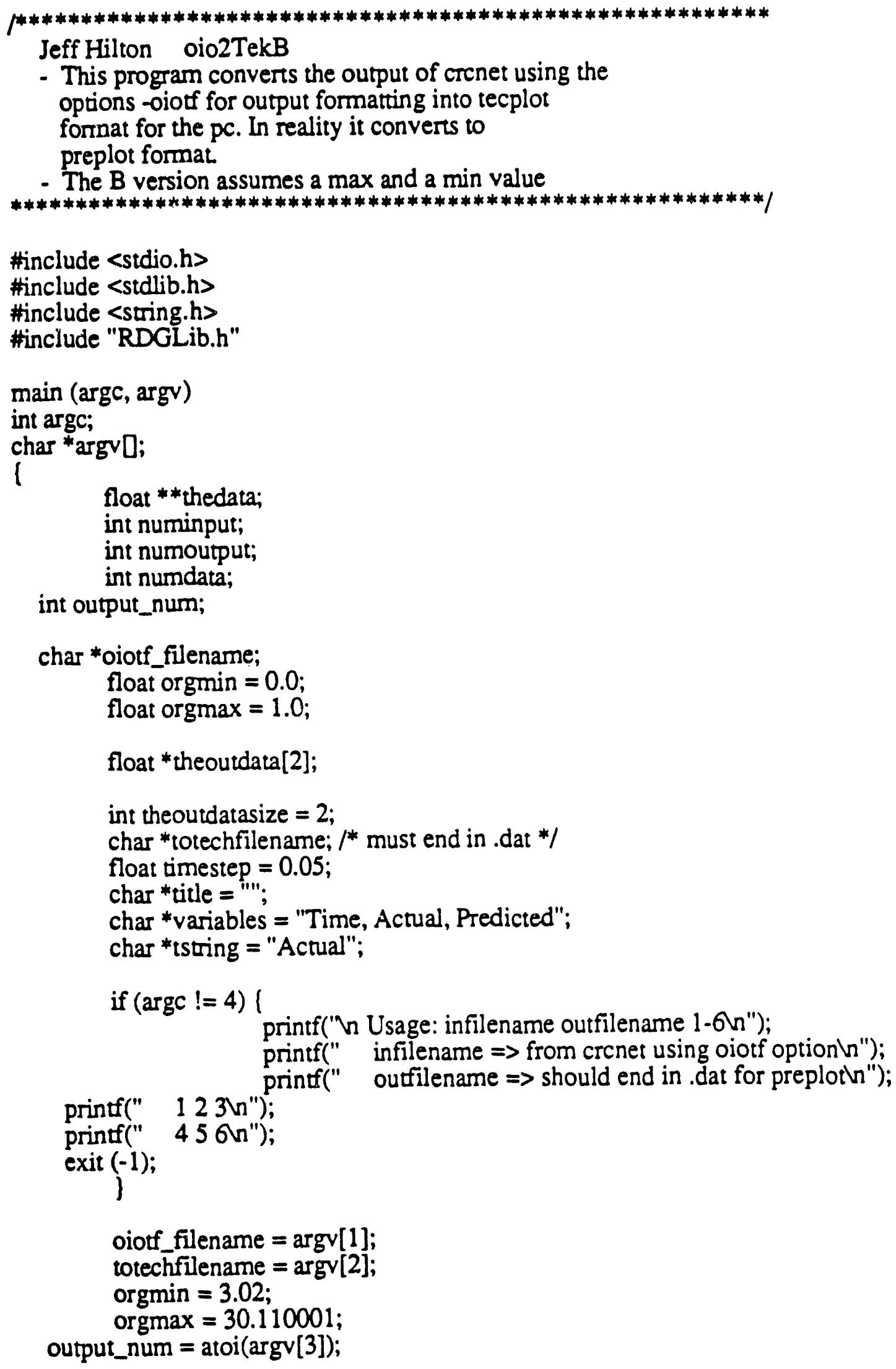

Jeff Hilton oio2TekB

- This program converts the output of crenet using the options -oiotf for output formatting into tecplot fornat for the pc. In reality it converts to preplot format.

- The $B$ version assumes a max and a min value $\neq * * * * * * * * * * * * * * * * * * * * * * * * * * * * * * * * * * * * * * * * * * * * * * * * * * * * * * * * 1$

\#include <stdio.h> \#include <stdlib.h> \#include <string.h> \#include "RDGLib.h"

main (argc, argv)

int argc;

char *argvD;

l

float **thedata;

int numinput;

int numoutput;

int numdata;

int output_num;

char *oiotf_filename;

float orgmin $=0.0$

float orgmax $=1.0$;

float *theoutdata[2];

int theoutdatasize $=2$;

char *totechfilename; /* must end in dat */

float timestep $=0.05$;

char *title =""';

char *variables = "Time, Actual, Predicted";

char *tstring = "Actual";

if $(\operatorname{argc} !=4)$ (

printf(" $\left.123 \mathrm{ln}^{\prime \prime}\right)$;

printf(" $\vee$ Usage: infilename outfilename 1-6n");

printf(" infilename => from crcnet using oiotf optionln");

print(" $456 \mathrm{Gn}$ ");

printf(" outfilename => should end in .dat for preplot $n "$ ");

exit $(-1)$;

oiotf_filename $=\operatorname{argv[1]}$;

totechfilename $=\operatorname{argv}[2]$;

orgmin $=3.02$;

orgmax $=30.110001$

output_num = atoi(argv[3]); 
read_oiotf_File (oiotf_filename, \& thedata, \&numinput, \&numoutput, \&numdata, orgmin, orgmax);

$/ *$ get the first of the training and the first of the output */

theoutdata[0] = thedata[numinput + numoutput + output_num - 1];

theoutdata[1] = thedata[numinput + output_num -1$]$;

write_TechPlot_File (totechfilename, theoutdata, theoutdatasize,

numdata, timestep, NULL, title, variables,

$$
\text { tstring); }
$$

1

deallocate_oiotf_Data (thedata, numinput, numoutput); 


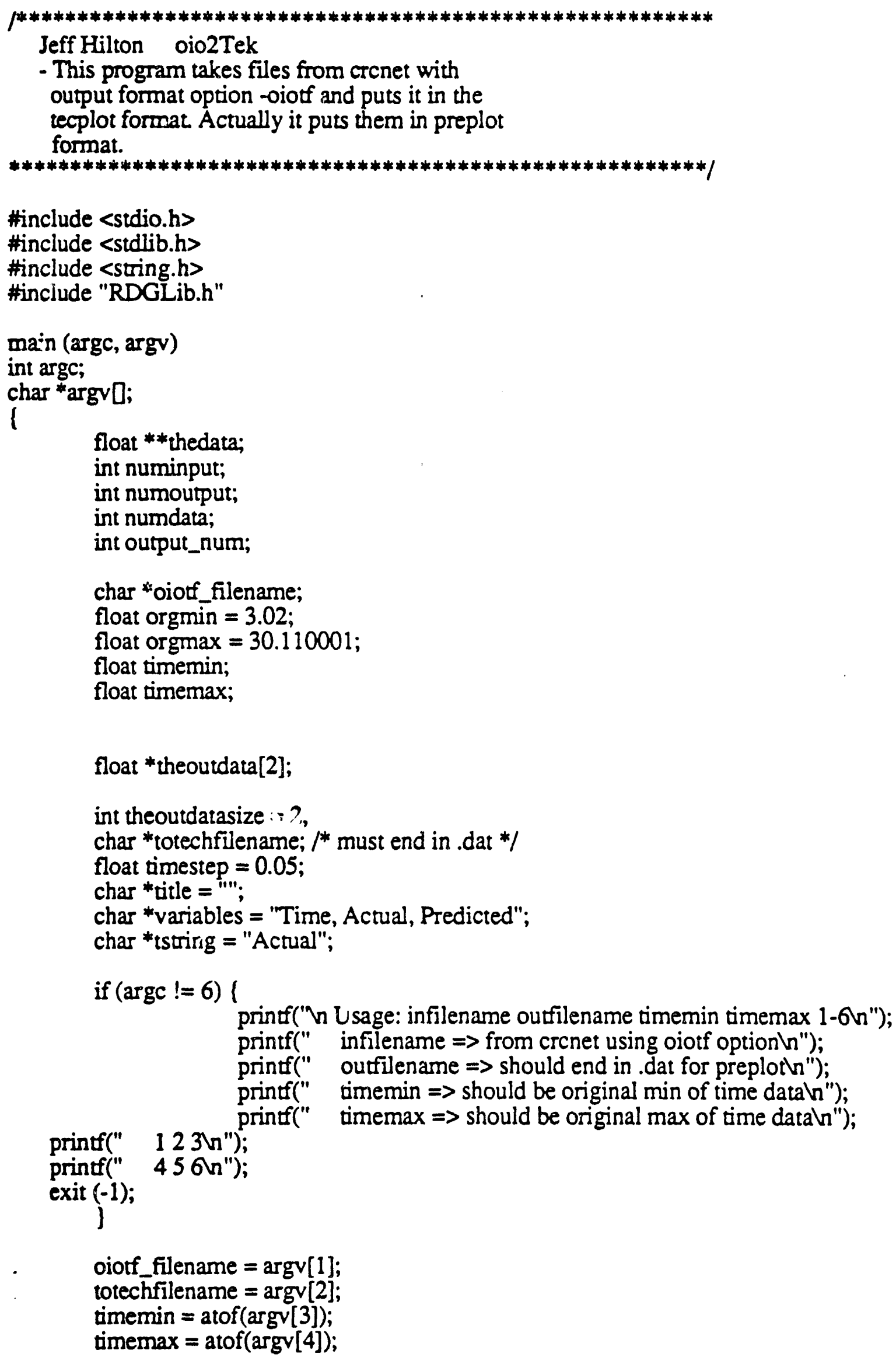


output_num = atoi(argv[5]);

read_oiotf_File (oiotf_filename, \&thedata, \&numinput, \&numoutput, \&numdata, orgmin, orgmax);

$/$ * unnnrm the time data but first renorm it to orgmin \& orgmax * normDataNP5ToPP5(thedata[0], numdata, orgmin, orgmax); unNormDataNP5ToPP5(thedata[0], numdata, timemin, timemax); $/ *$ convert time back from change in time */ convertFromChangeInTime (thedata[0], numdata);

$1^{*}$ get the first of the training and the first of the output */ theoutdata[0] = thedata[numinput + numoutput + output_num - 1];

theoutdata[1] = thedata[numinput + output_num - 1];

write_TechPlot_File (totechfilename, theoutdata, theoutdatasize, variables, numdata, timestep, thedata[0], title, tstring);

deallocate_oiotf_Data (thedata, numinput, numoutput); 
f***************************************************************

RDGLib.h Recurrent net Data Generator Library

Jeff Hilton

These routines are put here to transform the data

from the iar file (Interpolated Averaged \& Rounded) into

crenet format.

Thier are a lot of constants in the data!

Some start at 1 and some start at 0 .

I tried to keep them inclusive of the max and min values.

$* * * * * * * * * * * * * * * * * * * * * * * * * * * * * * * * * * * * * * * * * * * * * * * * * * * * * * * * * * * * * * * 1$

\#ifndef RDGLIB_H

\#define RDGLIB_H

\#include <stdio.h $\overline{>}$

\#define MIN_TMME $0 \quad / *$ Interpolated Averaged \& Rounded for 05 day*/

\#define MAX̄_TMME $7720 / *$ would be 3860 for iar .10 day *I

\#define MIN_WEEK 0

\#define MAX $\_$WEEK $139 / *$ would be ??? for iar .10 day */

\#define MIN_DEPTH $300 / * 300 \mathrm{~cm} * 1$

\#define MAX DEPTH 1800

\#define DEPTH_INC $30 / *$ depth was taken at $30 \mathrm{~cm}$ increments */

\#define MIN_DEPTH_NO $0 / *$ The number of depths */

\#define MAX_DEPTH_NO 50

\#define MIN_DNECTION $1 /$ * There were $\mathrm{n}$ injections */

\#define MAX_INJECTION 52 /* 1 a 7 day week for n weeks */

\#define DAYSINAWEEK 7 * Raw data starts at 0 and each injection */

\#define RAWDATASIZE 73 /* each raw data file has 73 columns *I

extern void getInjectionTime ();

extern void getMinMax ();

extern void normDataOTol ();

extern void unNormDataOTol ();

extern void NormDataNP5ToPP5 ();

extern void unNormDataNP5ToPP5 ();

extem int convertToDepthNum 0 ;

extern int readADepth ();

extern void takeLogOfData ();

extern void read_oiotf_File 0; 
extern void deallocate_oiotf_Data 0 ;

extern void write_TechPlot_File 0;

extern void getRawInjectionTime 0;

extern int readRawData();

extern int readRawDataWFill();

extern void interpData0;

extern void getAllMinMax 0;

extern void normAllData 0 ;

extern void write_crenet_File ();

extern float **makeArrayOfArrays();

extern void deallocateArrayOfArrays();

extern void fillTimes ();

extern void fillData ();

extern void convertToChangeInTime ();

extern void convertFromChangeInTime ();

extern int getSoilType ();

\#endif 


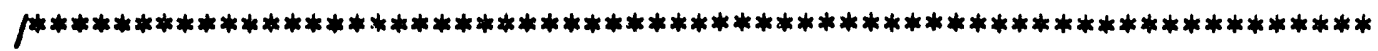

RDGLib.c Recurrent net Data Generator Library

Jeff Hilton

These routines are put here to transform the data

from one file format into another.

They support the following:

- iar files (Interpolated Averaged \& Rouneded) generated by either inter 05 or inter 10 .

- crenet (-oioff output, and input)

- preplot (tecplot)

- raw hanford data files

(X\#.dat; $X \Rightarrow A \cdot H ; \# \Rightarrow\{1,3,5,7\}$ or $(2,4,6,8\})$

THESE LIBRARIES ARE GROWING TOOLS

$* * * * * * * * * * * * * * * * * * * * * * * * * * * * * * * * * * * * * * * * * * * * * * * * * * * * * * * * * * * * * * * * * 1$

\#include <stdio.h>

\#include <stdlib.h>

\#include <string.h>

\#include <math.h>

\#include "RDGLib.h"

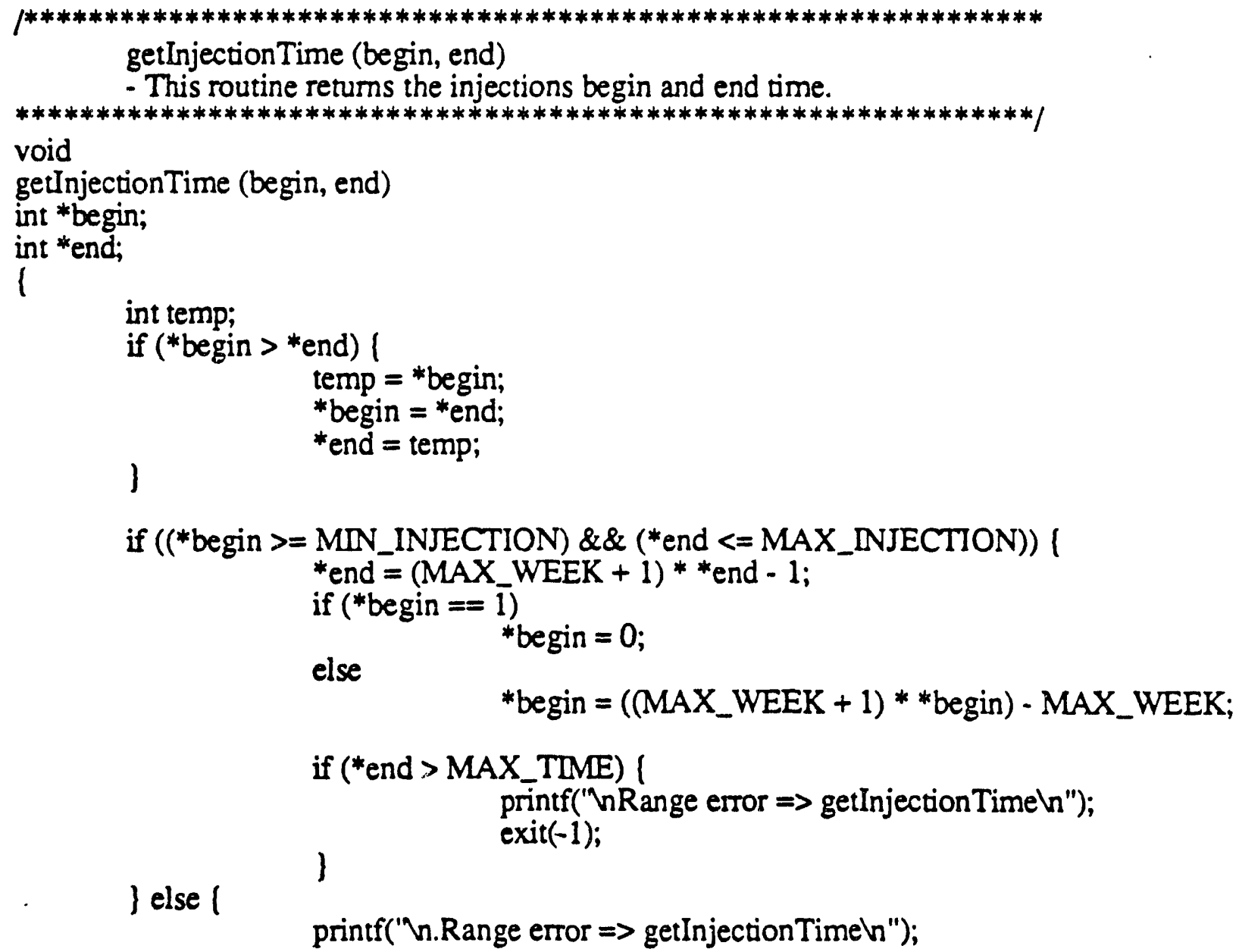




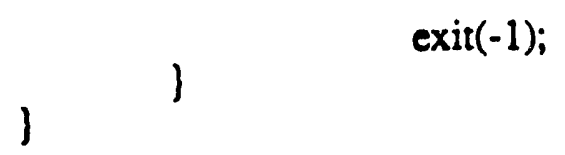

getMinMax (data, data_length, min, max)

- This routine retums the $\max$ and min values of the data

- It assumes that the max and min are previously set so that the user may get the max and min of several arrays.

- Assign max and min before the first time through this routine.

****************************************************************/

void

getMinMax (data, data_length, min, max)

float *data;

int data_length;

float *min;

float *max;

l

int index;

if $(($ data $=$ NULL $) \& \&$ (data_length $>0))$ ( printf (" nnNULI pointer error => getMinMaxh");

\} exit(-1);

for (index $=0$; index < data_length; index ++ ) \{

if (data[index] $<\min [0]$ ) $\min [0]=$ data $[$ index $]$

if (data[index] $>\max [0]$ ) $\max [0]=\operatorname{data}[$ index $]$

\}

)

/****************************************************************

normDataOTol (data, data_length, $\min , \max$ )

- norms data to range 0.0 to 1.0 over the entire data set

- assumes min and max are the min and max over the entire data set

- to be used with getMinMax

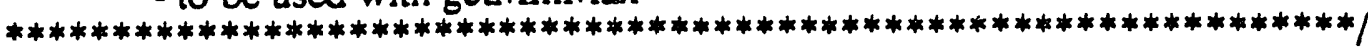

void

normData0Tol (data, data_length, $\min , \max$ )

float *data;

int data_length;

float min;

float max;

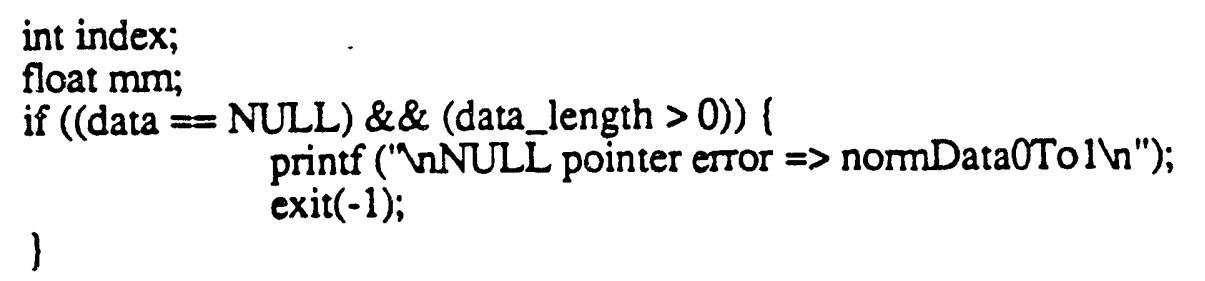




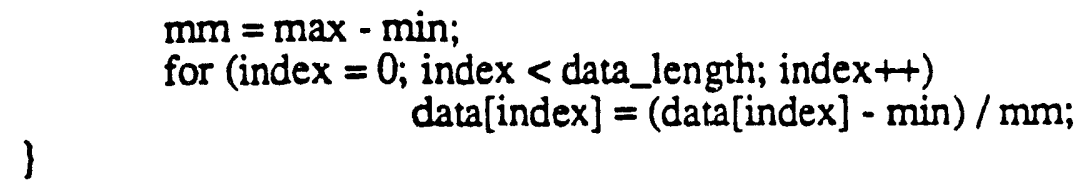




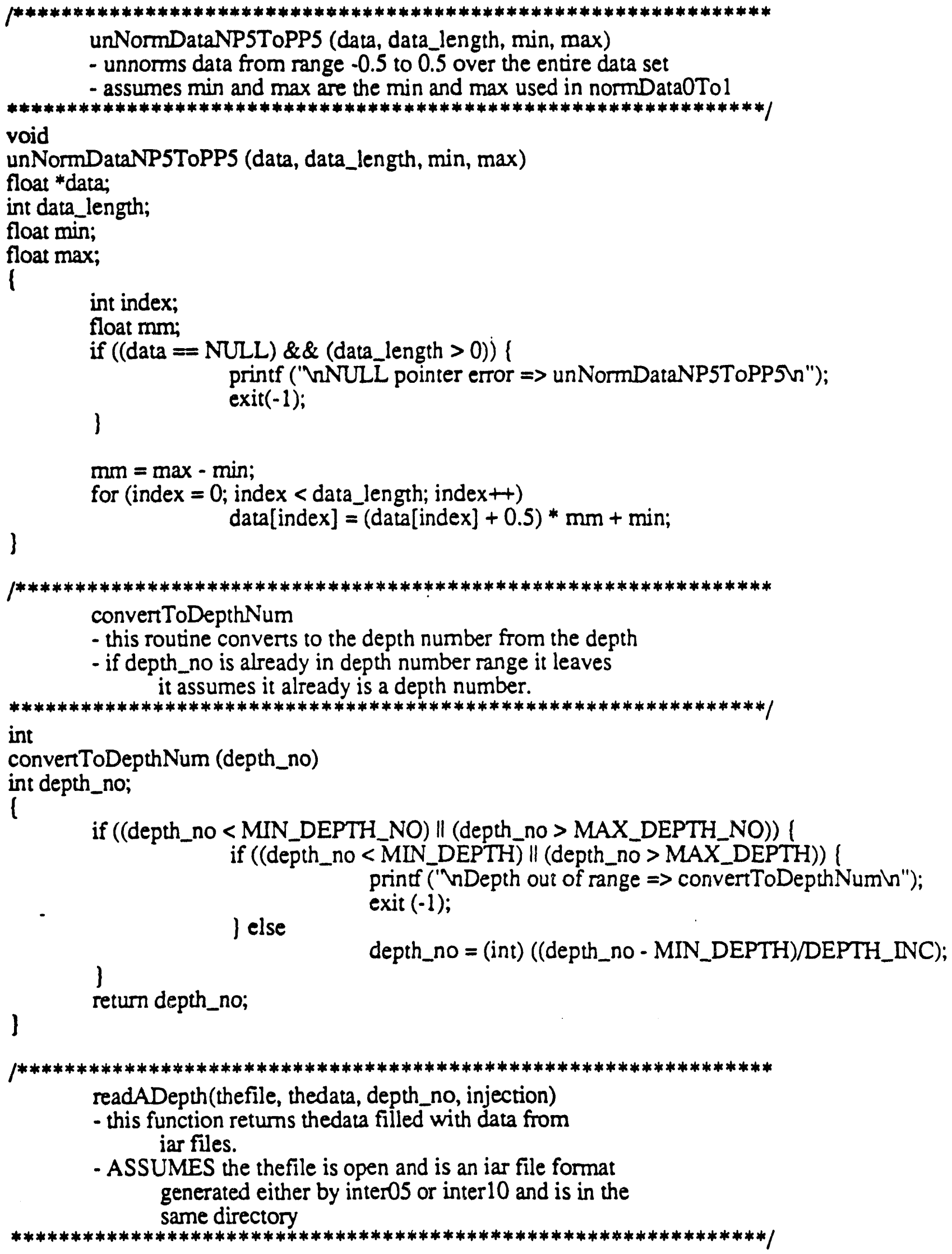




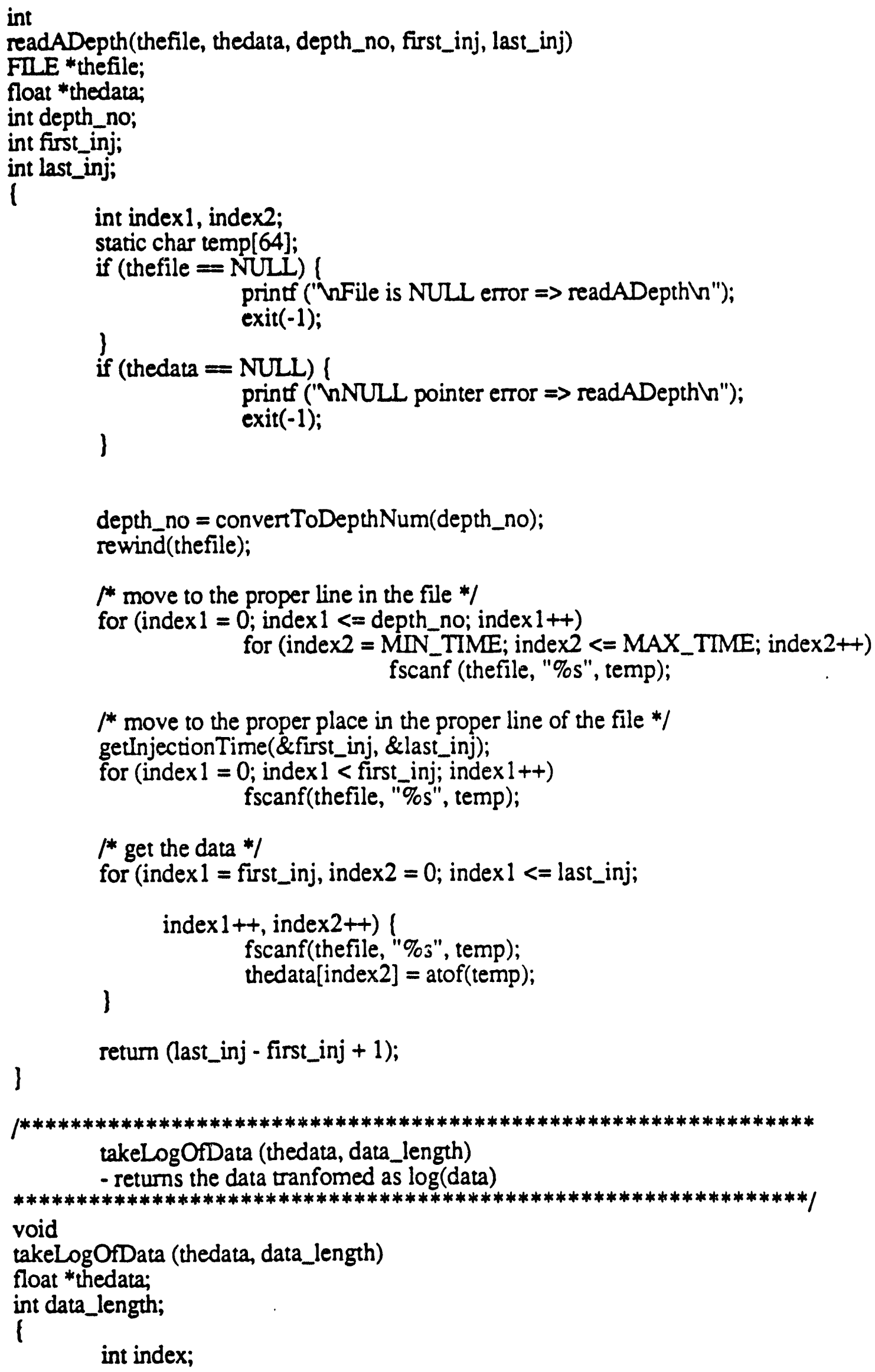




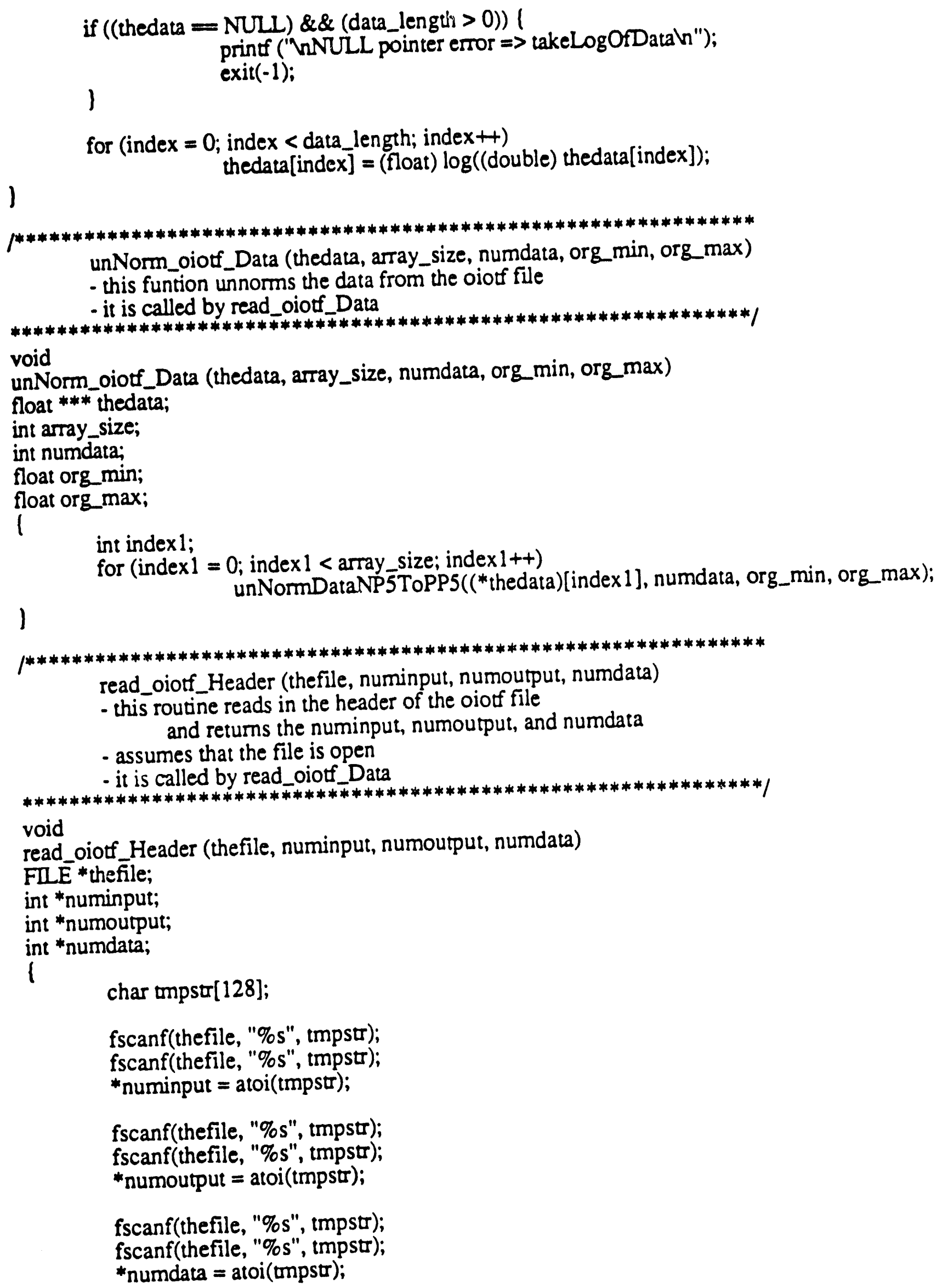

read_oiotf_Header (thefile, numinput, numoutput, numdata)

- this routine reads in the header of the oioff file

and returns the numinput, numoutput, and numdata

- assumes that the file is open

- it is called by read_oiotf_Data

***************************************************************1

void

read_oiotf_Header (thefile, numinput, numoutput, numdata)

FILE *thefile;

int *numinput;

int *numoutput;

int *numdata;

I

char tmpstr[128];

fscanf(thefile, "\%s", tmpstr);

fscanf(thefile, "\%s", tmpstr);

*numinput = atoi(tmpstr);

fscanf(thefile, "\%s", tmpstr);

fscanf(thefile, "\%s", tmpstr);

*numoutput = atoi(tmpstr);

fscanf(thefile, "\%s", tmpstr);

fscanf(thefile, "\%s", tmpstr);

${ }^{*}$ numdata = atoi(tompstr); 
void

read_oiotf_Data (thefile, thedata, numinput, numoutput, numdata)

FIIE *thefile;

float *** thedata;

int numinput;

int numoutput;

int numdata;

1

static char tmpstr[64];

int index 1, index 2, start, stop;

int arraysize;

arraysize $=2$ * numoutput + numinput;

$1 *$ allocate the arrays */

*thedata $=\left(\right.$ float $\left.{ }^{*}\right)$ malloc $($ sizeof $($ float $*) *$ arraysize $)$;

if $(*$ thedata $==$ NULL) $l$ printf("ViError allocating *thedata => read_oiotf_Data");

\} exit $(-1)$;

for (index $1=0$; index $1<$ arraysize; index $1++$ ) (

$(*$ thedata $)$ [index 1$]=\left(\right.$ float $\left.{ }^{*}\right)$ malloc(sizeof(float) * numdata $)$;

if $\left({ }^{*}\right.$ thedata $)[$ index 1$]==$ NULL $)$ l printf(" inError allocating *thedata[\%d]", index 1); printf("=> read_oiotf_Data");

) exit(-1);

\}

$\rho^{*}$ get the data */

for (index $1=0$; index $1<$ numdata; index $1++$ ) (

${ }^{*}$ get the input data */ fscanf(thefile, "\%s", tmpstr);

for (index $2=0$; index 2 <uminput; index $2++$ ) ( fscanf(thefile, "\%s", tmpstr);

)

(*thedata)[index2][index 1$]=$ atof(tmpstr);

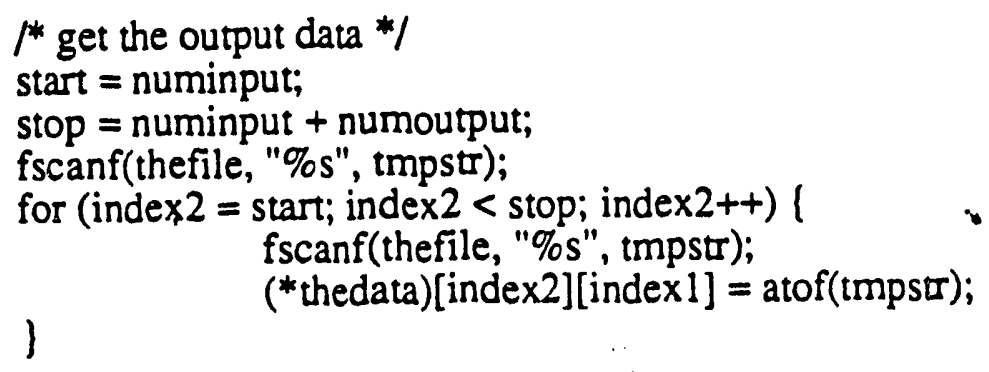




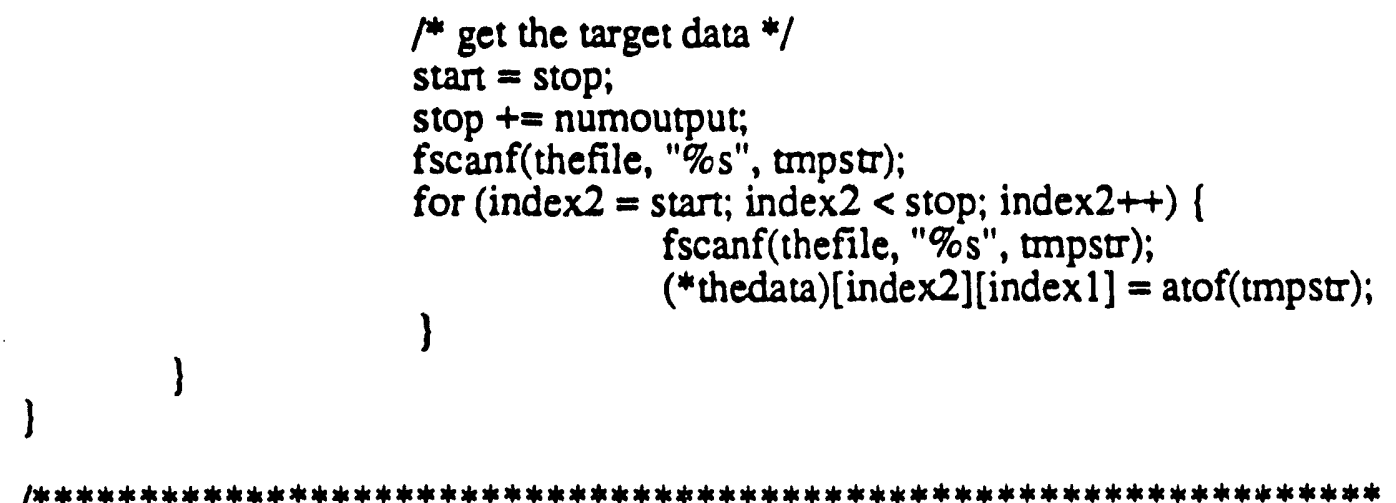

read_oiotf_File (thefilename, thedata, numinput, numoutput, numdata,

- this routine takes the name of an oiotf file generated org_min, org_max)

by rcmet using the -oiotf option and return the data in an array of arrays.

The arrays are in the form:

[input arrays.., output arrays.., target arrays..]

each of these array is numdata long

- Assumes that thedata will be allocated here and deallocated in deallocate_oiotf_Data outside this routine

- oiotf file format for inputs of 1, outputs of 5, and trains of 2

Numinputs: \# Numoutputs: \# Numtrain: \#

input: \#

output: \#\# \# \# \#

target: \#\#\#\#\#

input: \#

output: \# \# \# \# \#

target: \#\# \# \#

\footnotetext{
void

read_oiotf_File (thefilename, thedata, numinput, numoutput, numdata,

char *thefilename;

org_min, org_max)

float ***thedata;

int *numinput;

int *numoutput;

int *numdata;

float org min;

float org max;

l

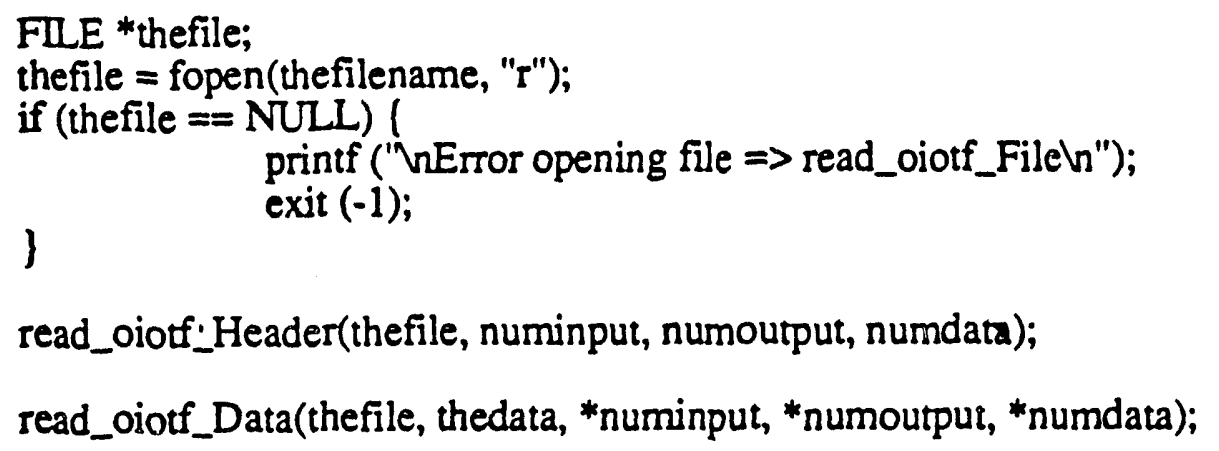




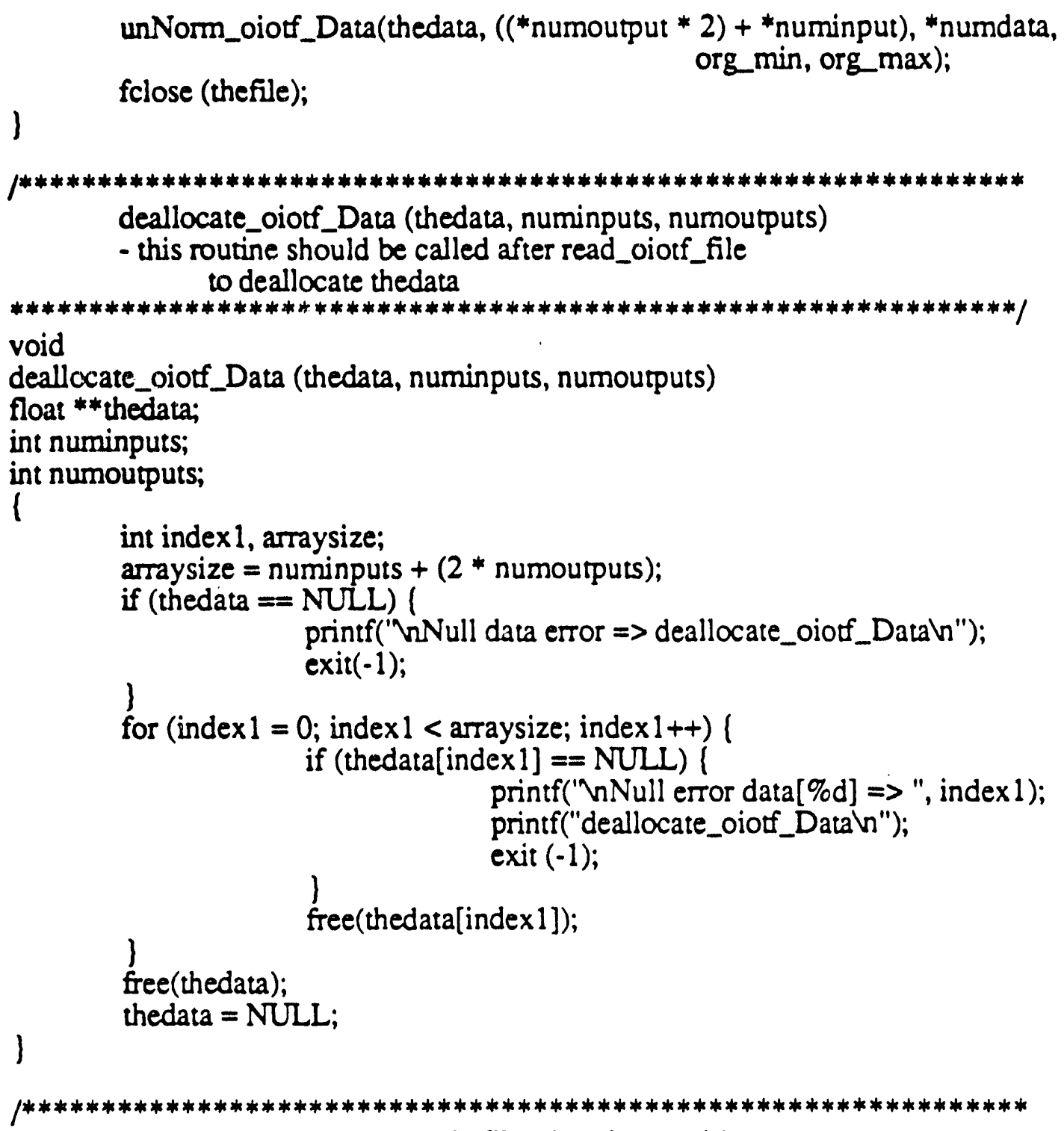

write_TechPlot_Header (thefile, sizeofarray, title,

- this routine writes the header for TechPlot data file

variables, tstring)

- called by write TechPlot_file

***************************************************************1

void

write_TechPlot_Header (thefile, sizeofarray, title,

FILE * thefile;

variables, tstring)

int sizeofarray;

char *title;

char *variables;

char *tstring;

fprintf (thefile, "TITLE = \"\%s\"ท", title);

fprintf (thefile, "VARIABLES = \%s n", variables);

fprintf (thefile, "ZONE T=l"\%s〉", ", tstring);

fprintf (thefile, "I = \%oln", sizeofarray); 
write_TechPlot_Data (thefile, thedata, numofarrays, sizeofarray,

- this routine writes out the data portion of a timestep, timedata) TechPlot file

- if the time data is NULL the time is set from

0 .. n by timestep increments

- called by write Tech Plot file

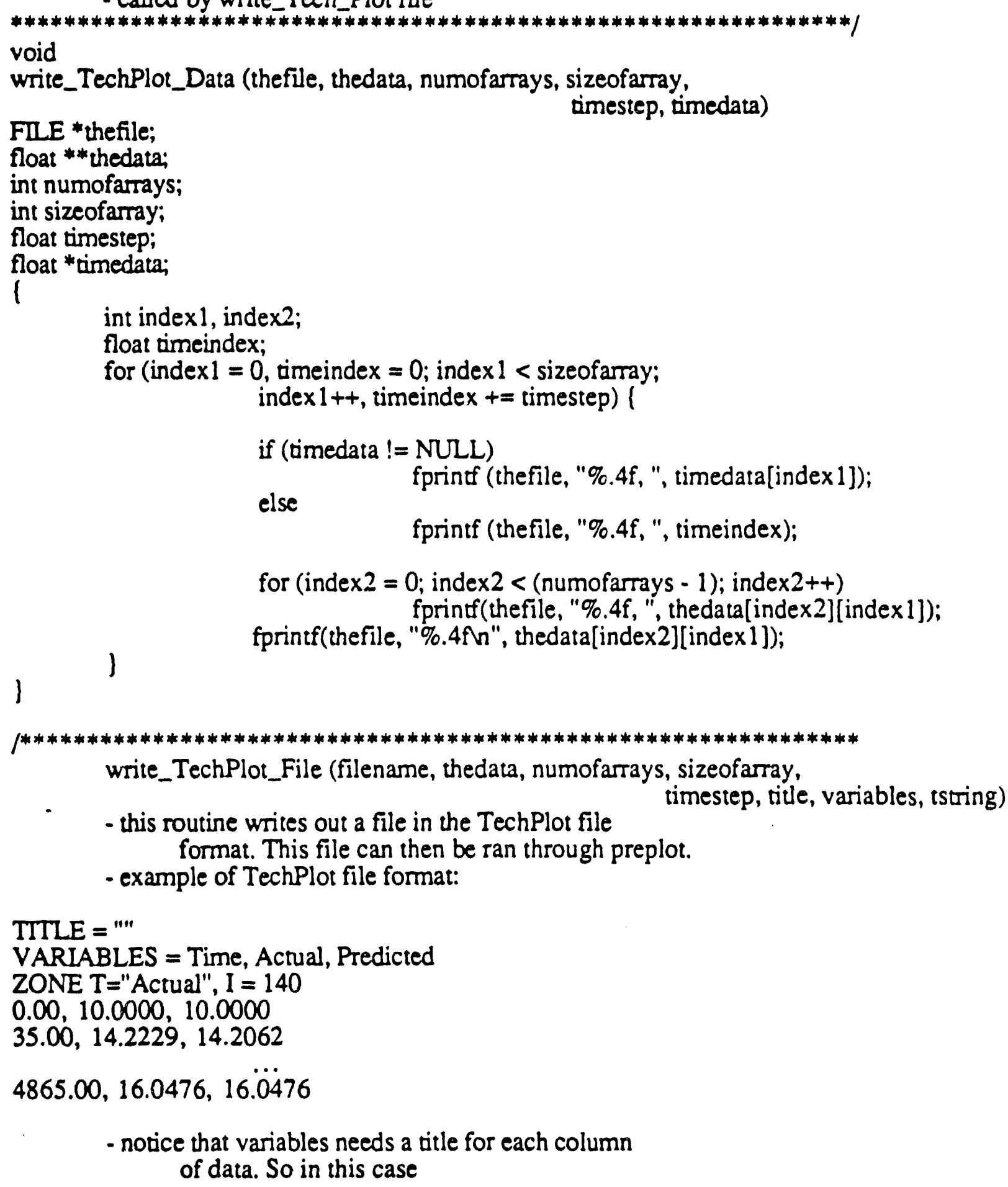


variable $=$ "Time, Actual, Predicted" and tstring = "Actual"

$* * * * * * * * * * * * * * * * * * * * * * * * * * * * * * * * * * * * * * * * * * * * * * * * * * * * * * * * * * * * * * * 1$

void

write_TechPlot_File (filename, thedata, numofarrays, sizeofarray,

char *filename;

timestep, timedata, title, variables, tstring)

float **thedata;

int numofarrays;

int sizeofarray;

float timestep;

float *timedata;

char * itle;

char *variables;

char *tstring;

l

FILE *thefile;

thefile = fopen (filename, "w");

if (thefile $==$ NULL)

printf ("UEError opening file => read_oiotf_Fileln");

) exit $(-1)$;

write_TechPlot_Header (thefile, sizeofarray, title, variables, tstring);

write_TechPlot_Data (thefile, thedata, numofarrays, sizeofarray,

fclose (thefile); timestep, timedata);

;

getLinearInterp $(x 1, y 1, x 2, y 2, x 3)$

- given two points and the $x$ for a third point

ASSUMED TO BE ONTHE SAME LNE AS THE FIRST TWO returns the $\mathrm{y}$ for this third point;

float

getlinearInterp $(x 1, y 1, x 2, v 2, x 3)$

float $x 1$;

float yl;

float $x 2$;

float $y 2$;

float $x 3$;

I

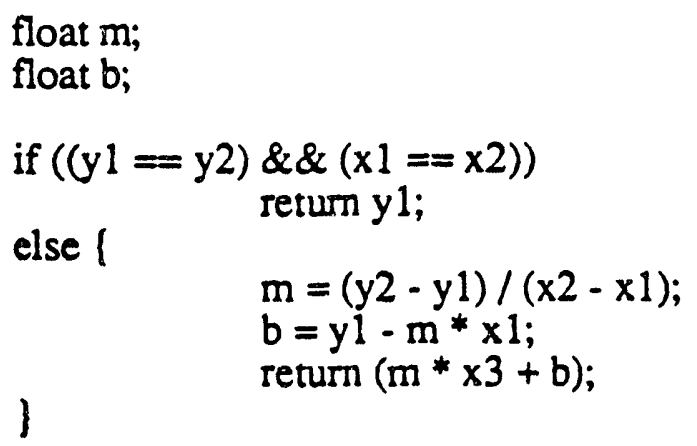


getBoundingTime(timedata, timedatasize, timewanted, $\mathfrak{t}, \mathrm{t} l$ )

- this routine finds the two points that bounding the time in timewanted and retums their index

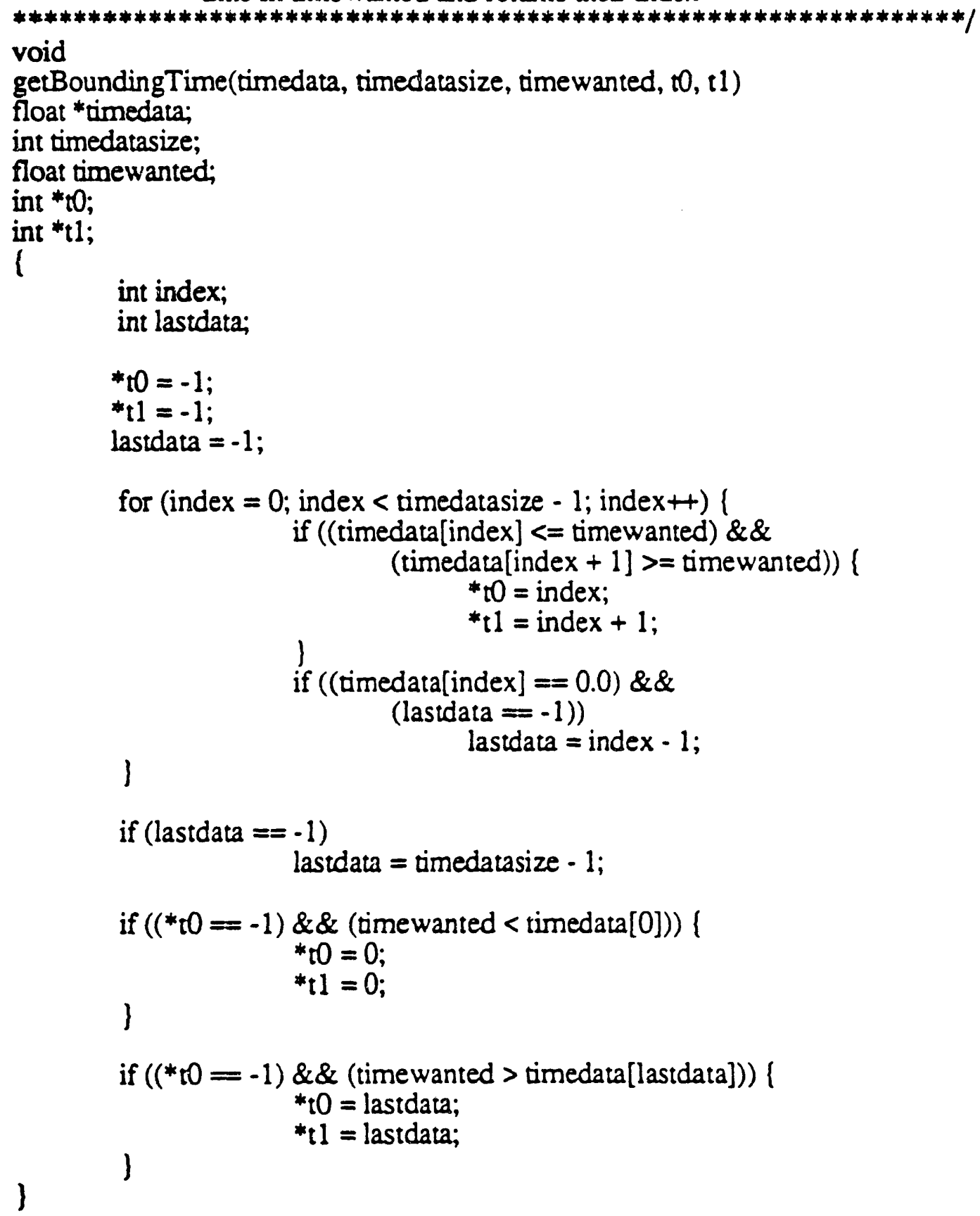

- This routine take the data and timedata and retums newdala and newtimedata for the increments sizes.

- ASSUMES that newdata and newdatasize are allocated arrays 
void

interpData(data, timedata, datasize, newdata, newtimedata, newdatasize,

float *data;

increment, timestar, timeend)

float *timedata;

int datasize;

float *newdata;

float *newtimedata;

int *newdatasize;

float increment;

float timestart;

float timeend;

l

float time;

int $t 0, t 1$, index;

if $(($ data $=$ NULL $) \|($ timedata $=$ NULL $) \|$

(newtimedata $==$ NULL) $\|($ newdata $==$ NULL $)$ )

printf ("Vn NULL data error ");

if $($ data $==$ NULL $)$

printf ("data ");

if (timedata $==$ NULL)

prinff ("timedata ");

if (newdata $==$ NULL)

printf ("newdata ");

if (newtimedata $==$ NULL)

printf(" $=>$ interpData");

printf ("newtimedata ");

\}

exit(-1);

for (index $=0$, time $=$ timestart, t $0=-1$; time $<=$ timeend;

time $+=$ increment) (

getBoundingTime (timedata, datasize, time, \& $\& 0, \& t 1)$;

if $((t 0>=0) \& \&(t 1>=0))$ newtimedata[index] = time; newdata[index] = getLinearInterp (timedata[t0], data[t0],

timedata[t]], data[t]],

time);

index+r;

)

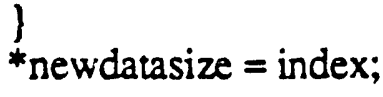

\}

l***************************************************************

getRawInjectionTime (first_inj, last_inj)

- this routine gets the injections in number (1..n) and returns in days as miltiples of week.

- called by ReadRaw

$* * * * * * * * * * * * * * * * * * * * * * * * * * * * * * * * * * * * * * * * * * * * * * * * * * * * * * * * * * * * * * *)$

void

getRawInjectionTime (first_inj, last_inj) 
int *first_inj;

int *last_inj;

1

$$
\text { int temp; }
$$

if (*first_inj > *last_inj) (

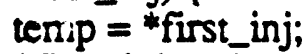

*first_inj = *last_inj;

1

*last_inj = temp;

*first_inj = (*first_inj * DAYSINAWEEK) - DAYSINAWEEK;

了

*last_inj = (*last_inj * DAYSINAWEEK);

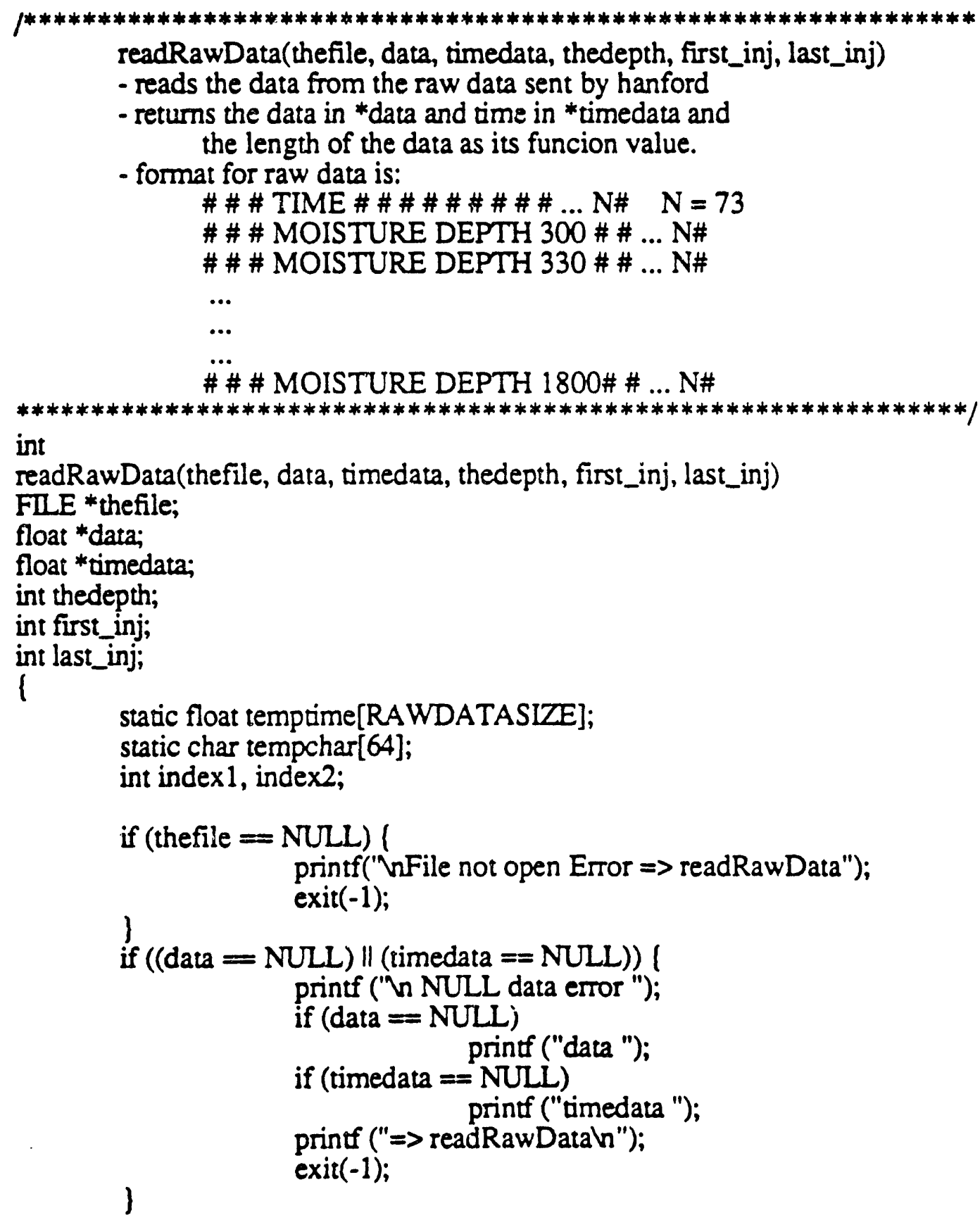

readRawData(thefile, data, timedata, thedepth, first_inj, last_inj)

- reads the data from the raw data sent by hanford

- returns the data in *data and time in *timedata and the length of the data as its funcion value.

- format for raw data is:

\# \# \# TIME \#\# \#\#\#\#\#\#\# \#... N\# $\mathrm{N}=73$

\#\# \# MOISTURE DEPTH 300 \#\# ... N\#

\# \# \# MOISTURE DEPTH 330 \#\# ... N\#

...

$\cdots$

\#\# \# MOISTURE DEPTH $1800 \#$ \# ... N\# 


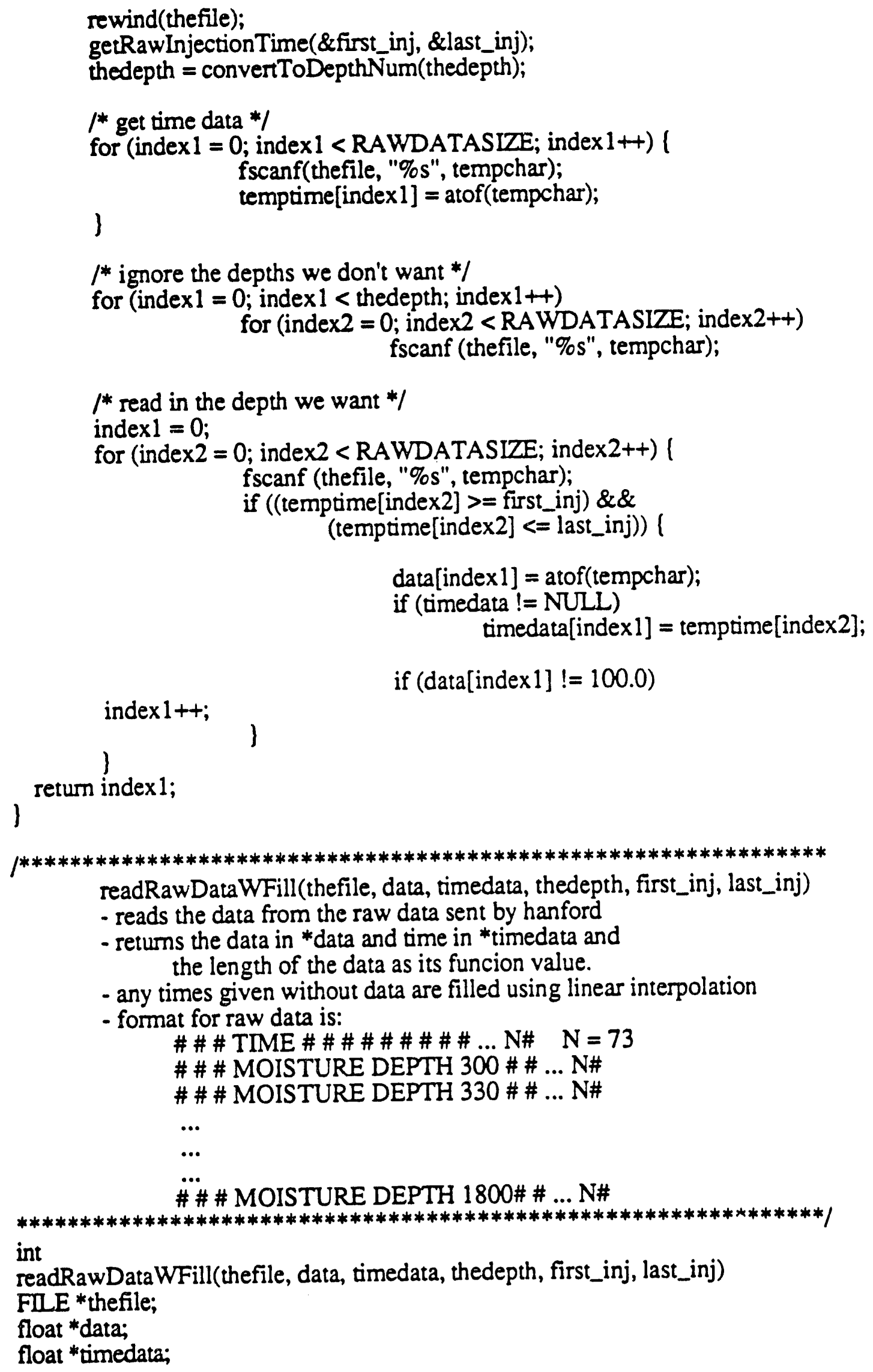

readRawDataWFill(thefile, data, timedata, thedepth, first_inj, last_inj)

- reads the data from the raw data sent by hanford

- returns the data in *data and time in *timedata and the length of the data as its funcion value.

- any times given without data are filled using linear interpolation

- format for raw data is:

\# \# \# TIME \# \# \# \# \# \# \# \# \# ... N\# $\mathrm{N}=73$

\# \# \# MOISTURE DEPTH 300 \# \# ... N\#

\# \# \# MOISTURE DEPTH 330 \# \# ... N\#

$\cdots$

$\cdots$

\#\# \# MOISTURE DEPTH 1800\# \# ... N\#

int

readRawDataWFill(thefile, data, timedata, thedepth, first_inj, last_inj)

FILE * thefile;

float *data;

float *imedata; 


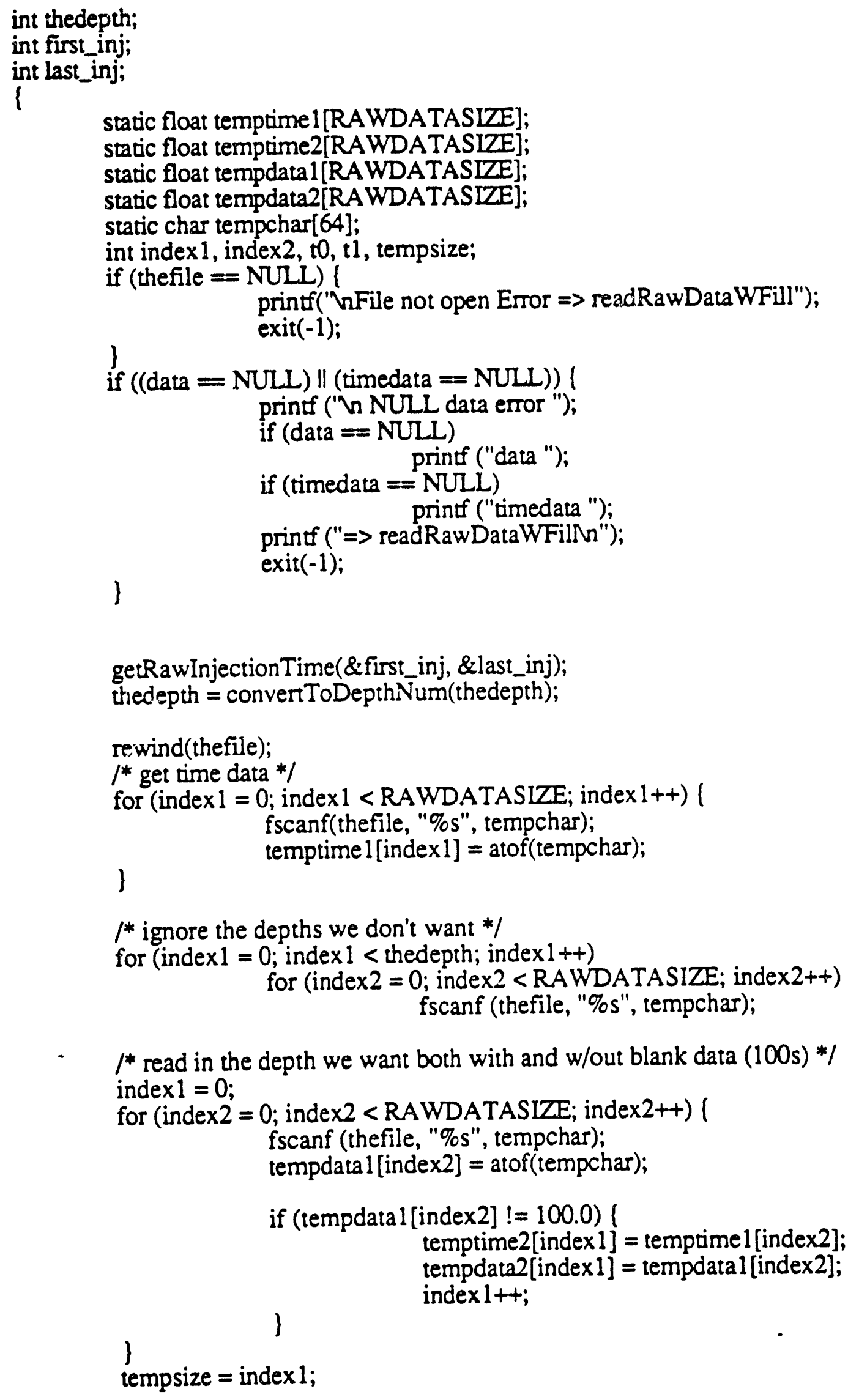




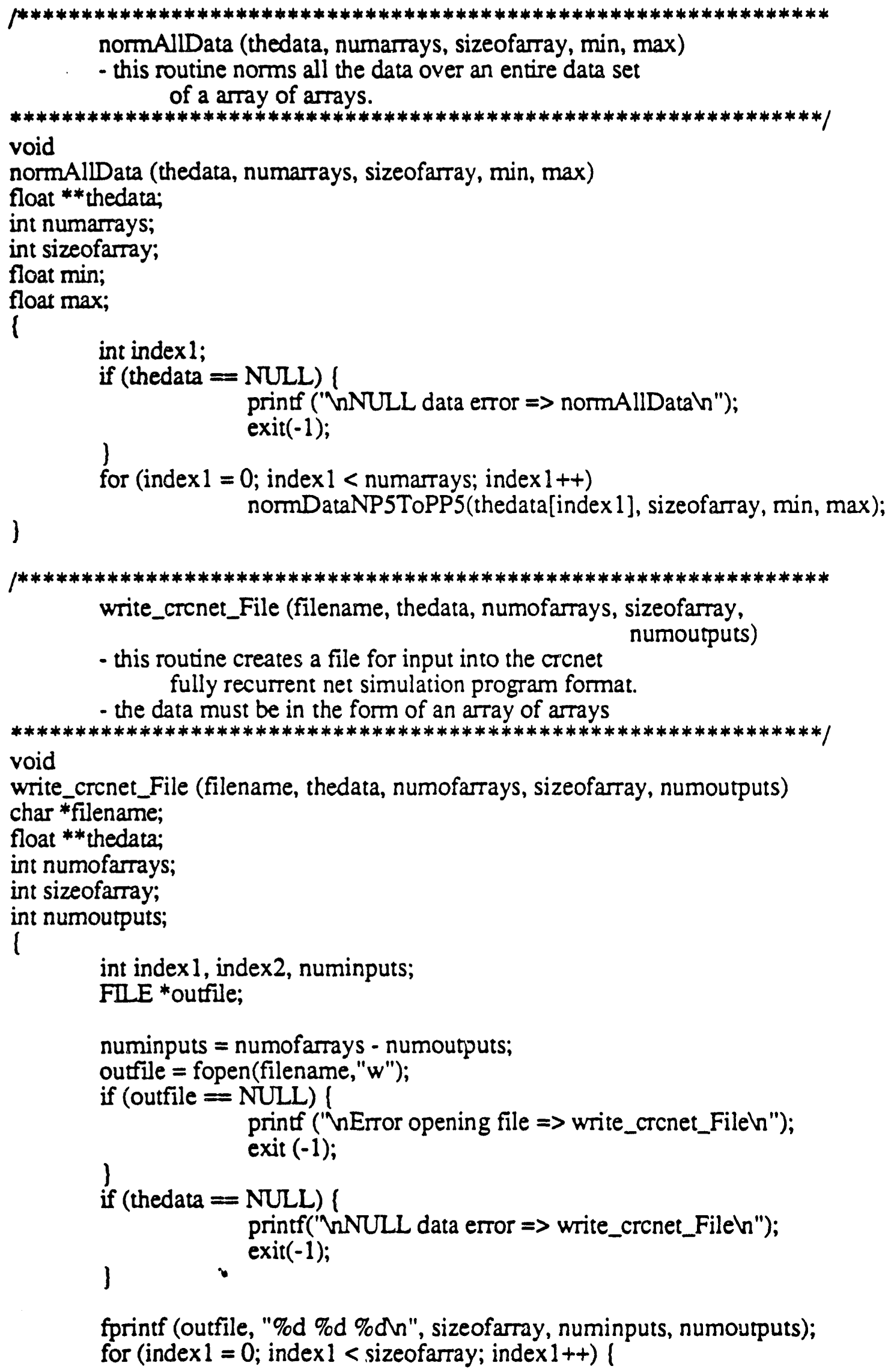

write_crenet_File (filename, thedata, numofarrays, sizeofarray,

- this routine creates a file for input into the cronet numoutputs)

fully recurrent net simulation program format.

- the data must be in the form of an array of arrays

$* * * * * * * * * * * * * * * * * * * * * * * * * * * * * * * * * * * * * * * * * * * * * * * * * * * * * * * * * * * * * * * 1$

void

write_crcnet_File (filename, thedata, numofarrays, sizeofarray, numoutputs)

char ${ }^{*}$ filename;

float **thedata;

int numofarrays;

int sizeofarray;

int numoutputs; l

int index 1 , index 2 , numinputs;

FIIE *outfile;

numinputs = numofarrays - numoutputs;

outfile = fopen(filename,"w");

if (outfile $==$ NULL) printf ("nError opening file => write_cronet_Fileh");

) exit (-1);

fprintf (outfile, "\%d \%d \%dn", sizeofarray, numinputs, numoutputs); for (index 1 = 0 ; index 1 < sizeofarray; index $1++$ ) ( 


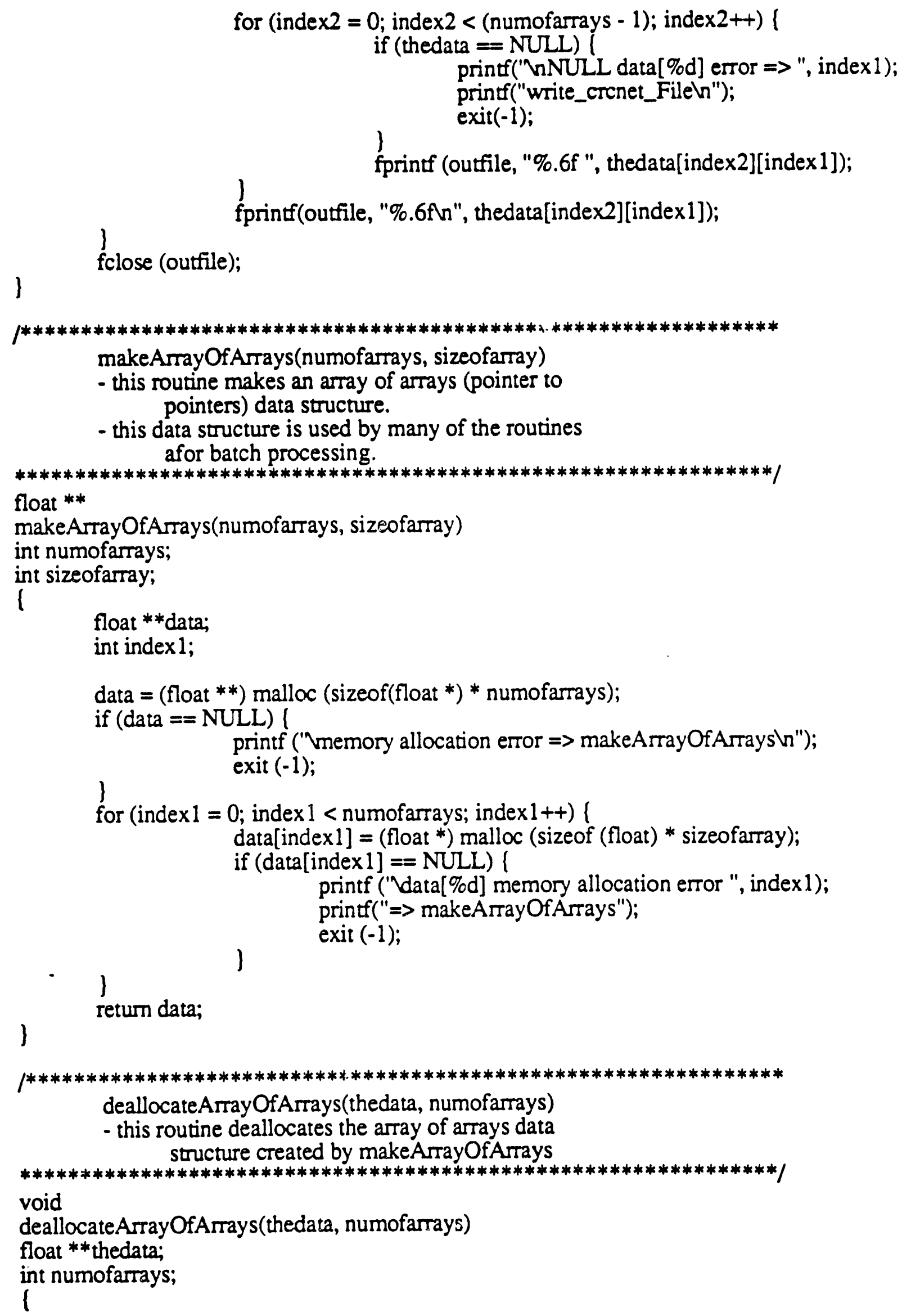

\section{I}

deallocateArrayOfArrays(thedata, numofarrays)

- this routine deallocates the array of arrays data structure created by makeArrayOfArrays

$* * * * * * * * * * * * * * * * * * * * * * * * * * * * * * * * * * * * * * * * * * * * * * * * * * * * * * * * * * * * * * *)$

void

deallocateArrayOfArrays(thedata, numofarrays)

float **thedata;

int numofarrays;

\{ 


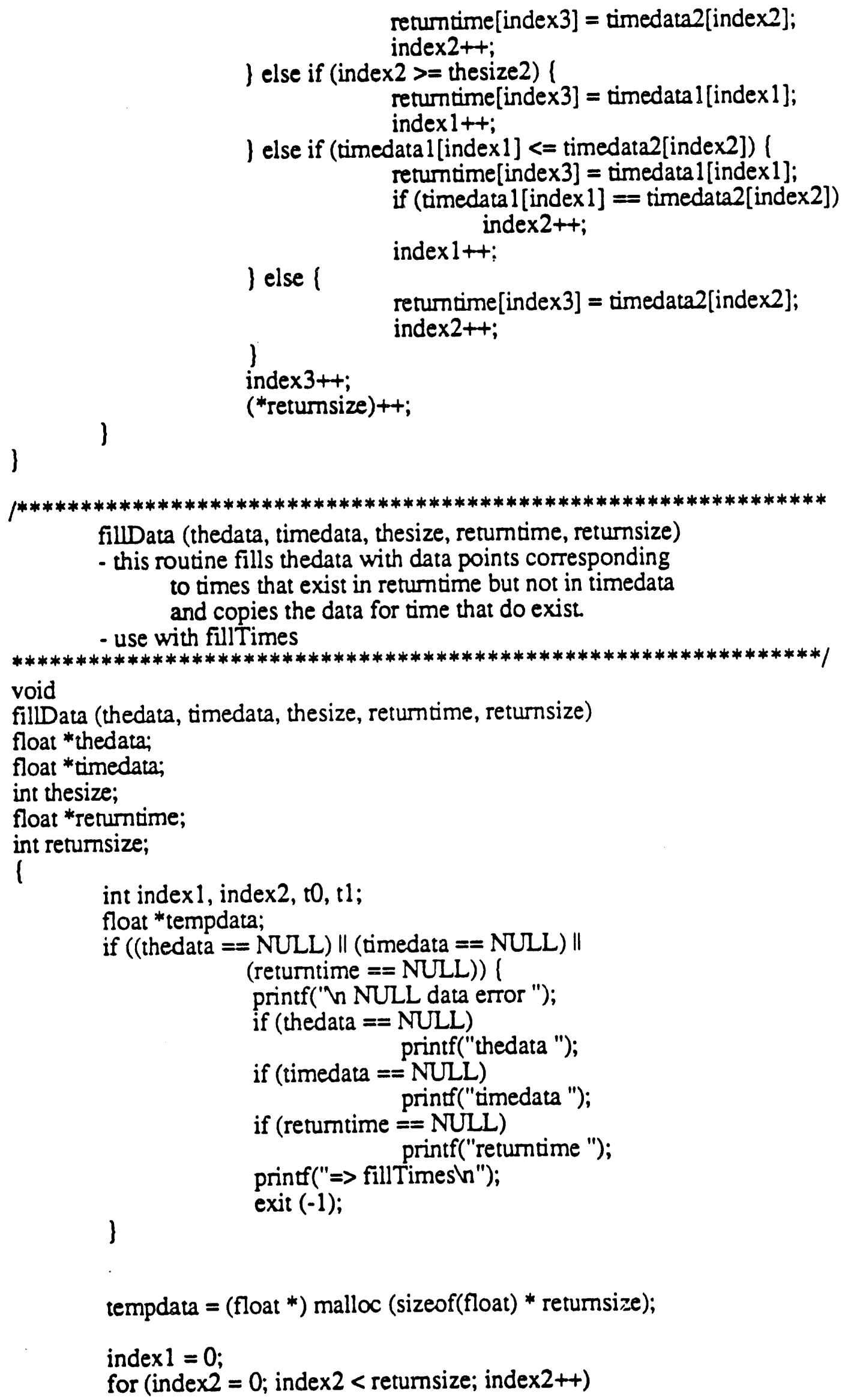




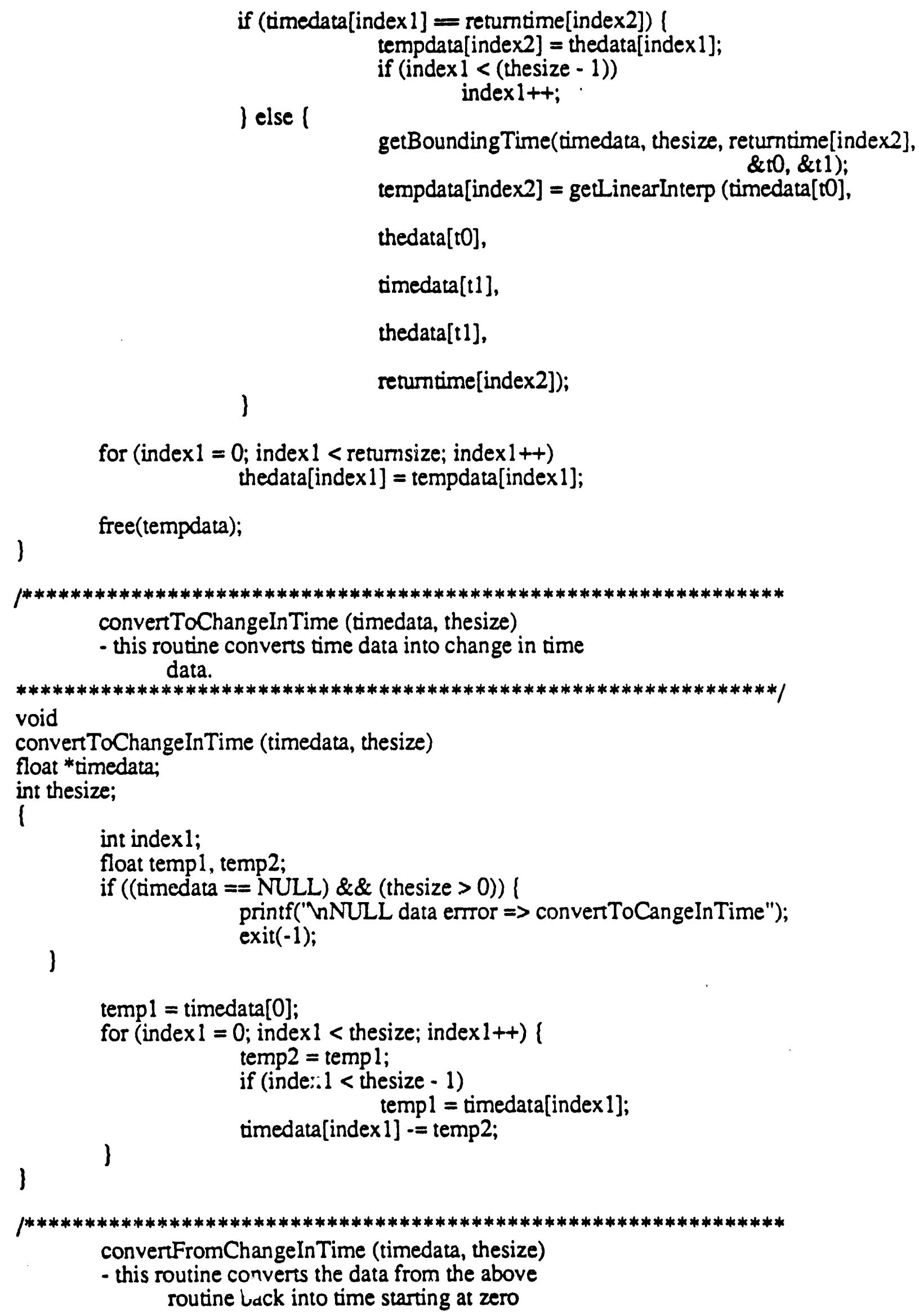




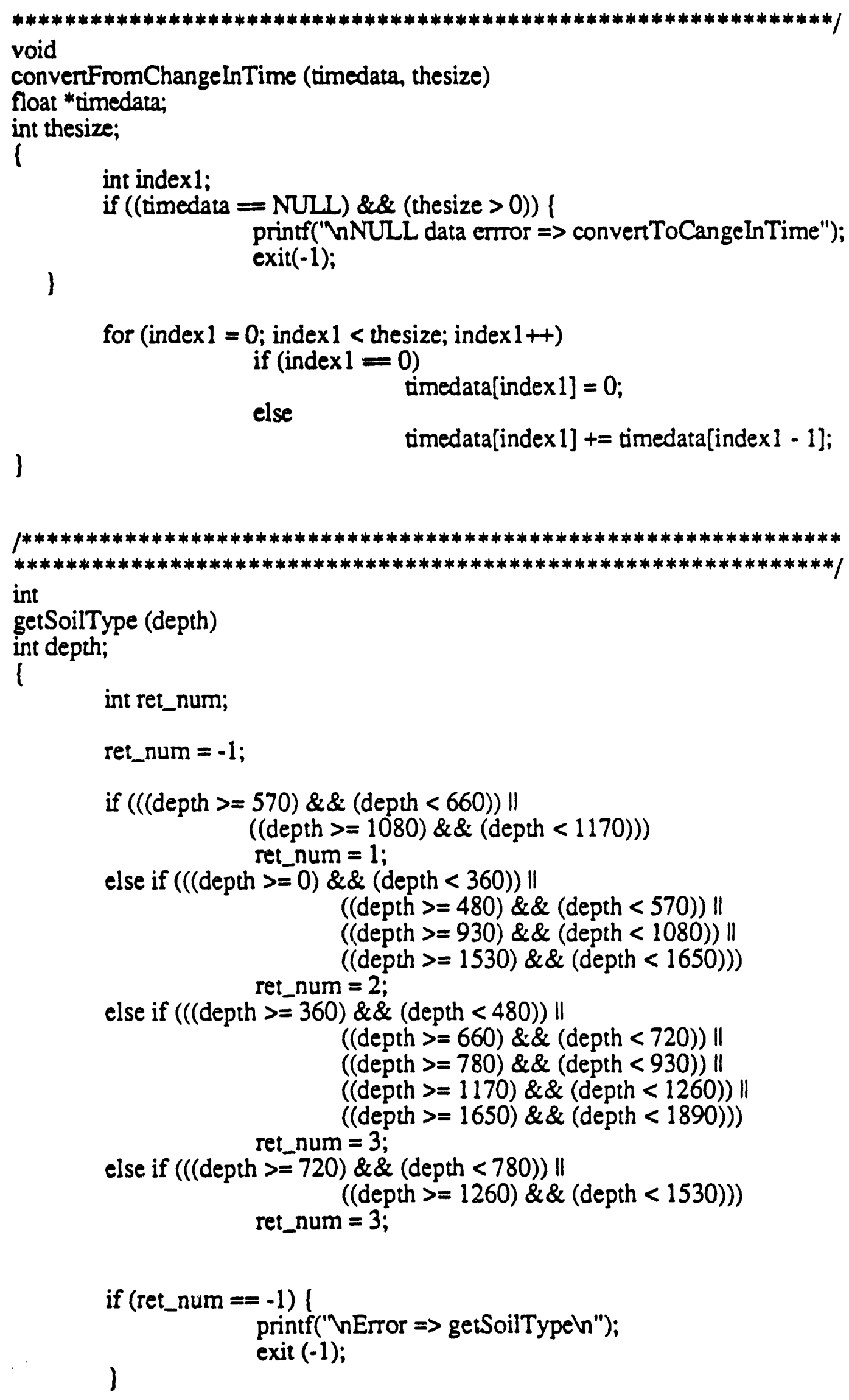




\section{\}}

return ret_num; 


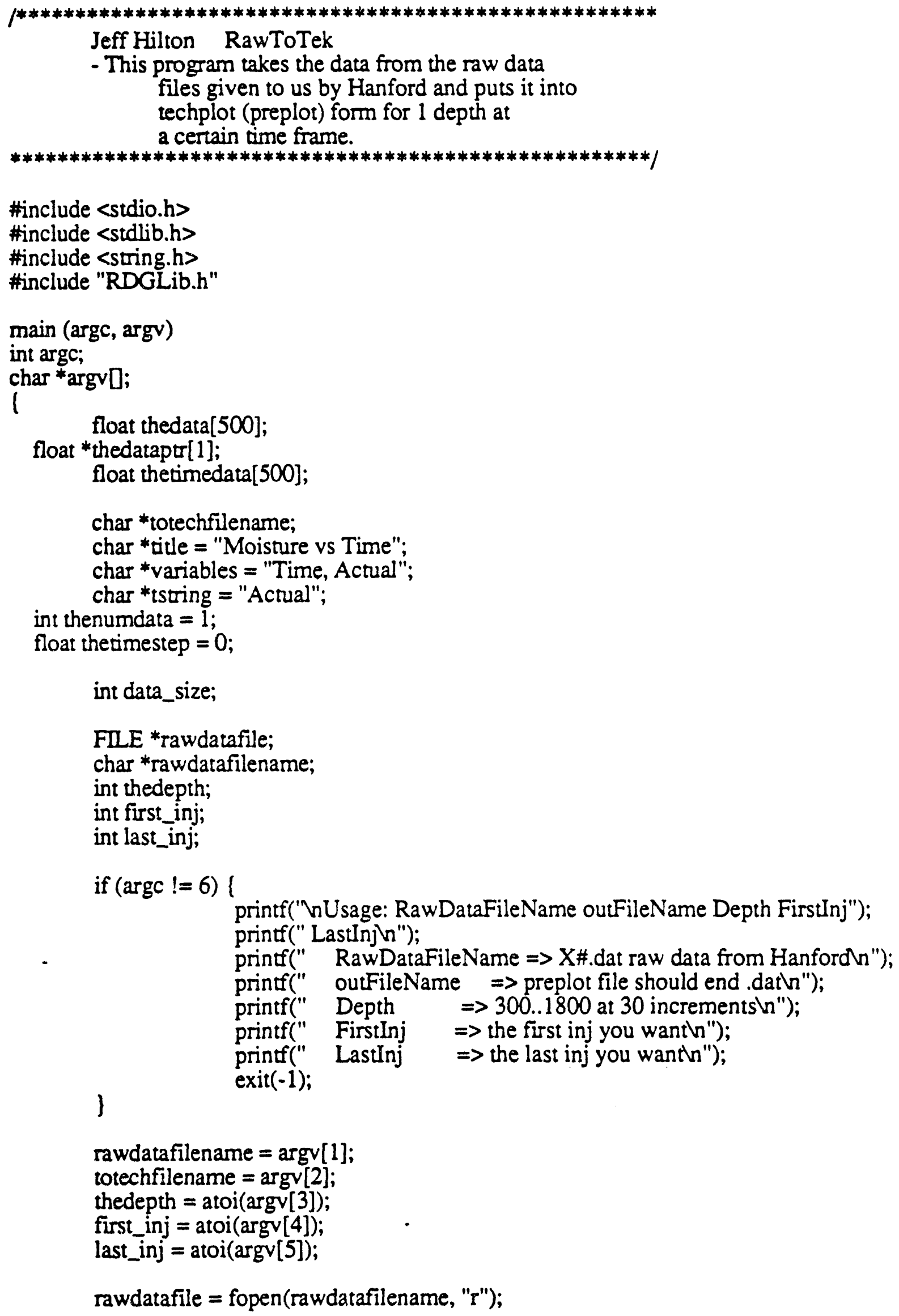




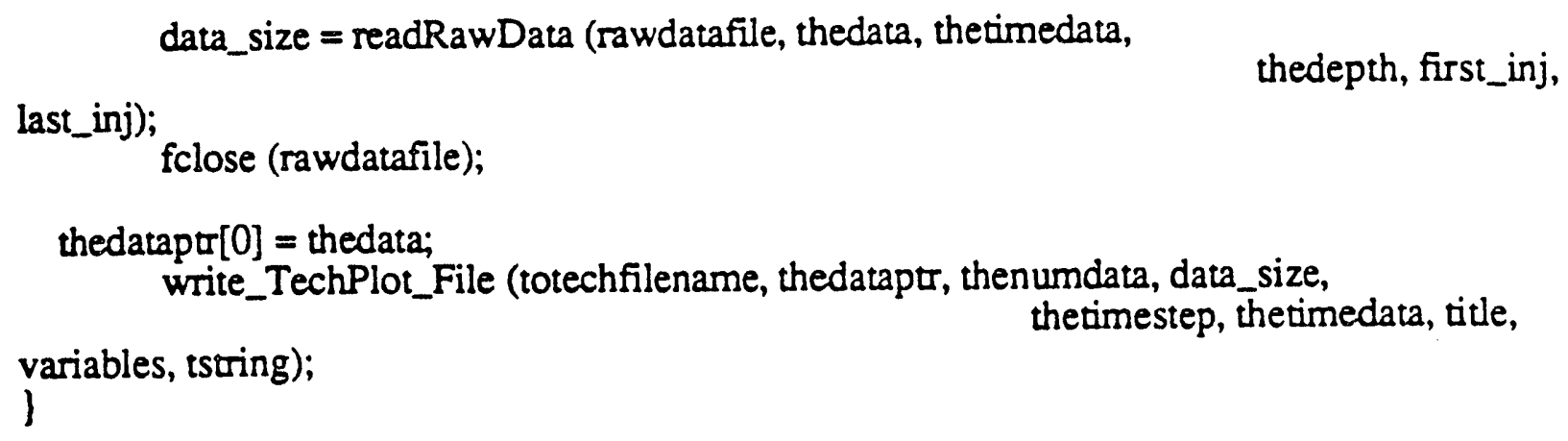


Appendix B

Back Propagation Network Process Development 
B-2 


\section{Appendix B}

\section{Back Propagation Network Process Development}

The back propagation network is an effective mapping function if the relationship between the input activation vector and output activation vector is a continuous nonlinear mapping. Applying the network to data indicates the degree of direct correlation between the input and output vector values. This methodology was used to evaluate the moisture data collected at the test site. Various networks were developed using subsets of the data to determine the completeness of the data and its general application to site screening.

Since the present data are sparse, a three-dimensional model, which would be the most useful and accurate model, could not be trained to any reasonable precision. A two-dimensional model, however, was a good approximation. The effective moisture values for the two-dimensional model were obtained at each depth from the surface and distance from the injection, by averaging the measured values over radial directions. This reduced the space to two dimensions, depth and distance from the source. Each cell borders on four others, so that the model predicted the moisture at time, $t+1$, for a cell based on itself and its neighbors moisture at time, $t$.

The site was broken down into grid cells along the vertical and horizontal axes. The size of the grid cells were $200 \mathrm{~cm}$ horizontally and $30 \mathrm{~cm}$ vertically. The grid cell approach requires moisture measurements to be recorded over time intervals at all grid cell locations within the site. The data provided by Westinghouse Hanford Company were not recorded in this manner. The moisture measurements were recorded at varying times, mainly during periods of large moisture changes, and measurements were not recorded at all locations, again only where large moisture changes were observed. Decision was made to interpolate the data between times when moisture data were available as shown in Figure B-1. A time interval of 0.05 days was chosen to accurately represent the moisture changes especially the moisture spikes. Depths and locations containing no measured moisture values over the entire experiment were considered to be at background levels. Following the interpolation, the cell networks were trained on the following inputs:

- Moisture values for five grid cells. One representing the current moisture of the locations to predict the moisture change for and the other four representing the adjacent horizontal and vertical locations.

- Hydraulic conductivity for the five grid cells that depends on the soil type.

The networks were trained to predict the change in moisture from time, $t$, to time, $t+0.05$ days for the central grid cell. The network was unable to accurately learn.

The analysis of the data revealed two problems that could have resulted in the network's inability to learn. The first problem had to do with the magnitude of the moisture change. The training data were comprised mainly of zero moisture changes (90\% of the data) because of the moisture values being at or near background values. This caused the network to produce zero moisture change in all cases. This problem was solved by grouping the moisture changes into sets 


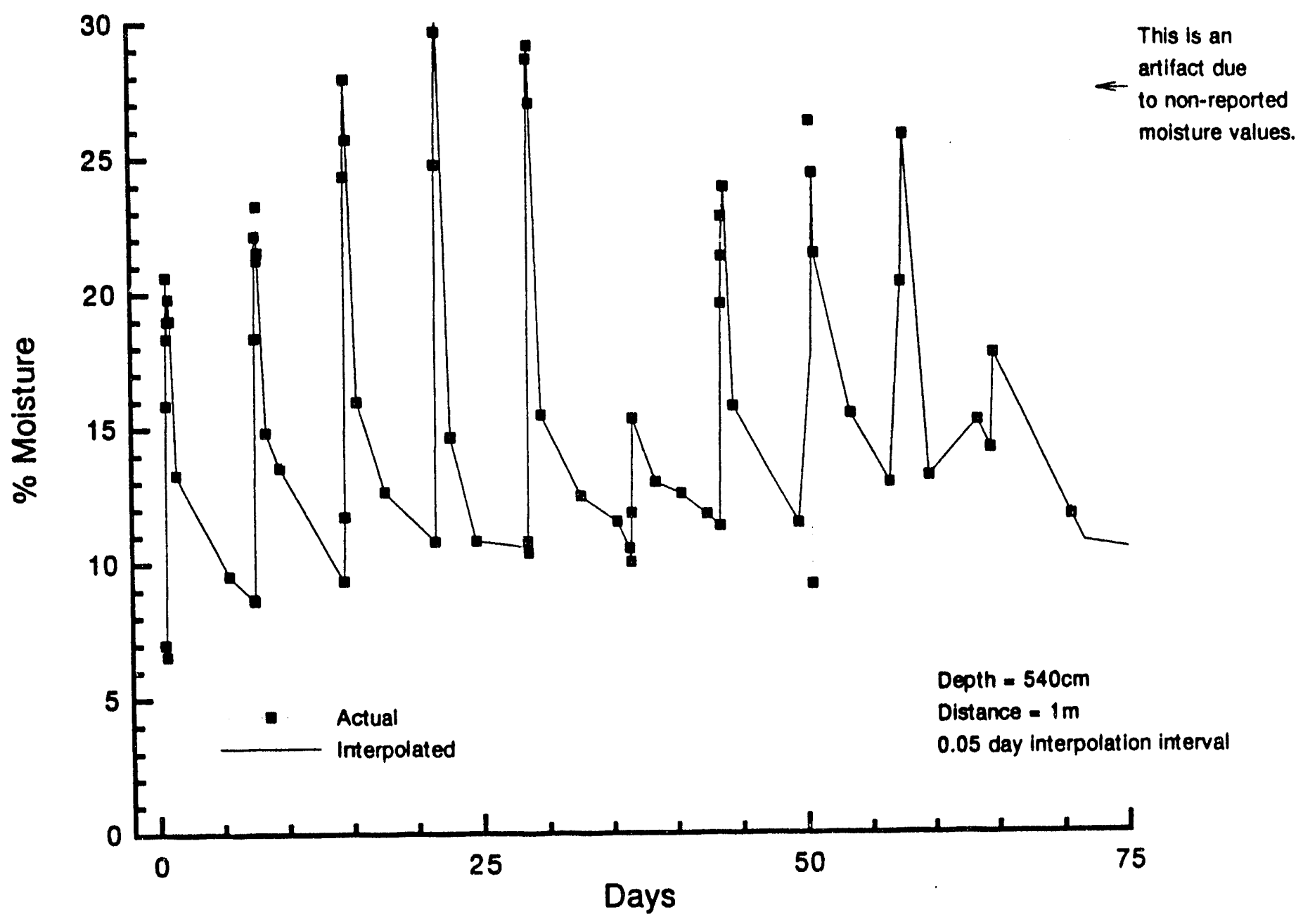

Figure B-1. Actual versus interpolated moisture values. 
with this data. The network had 10 hidden units and was trained for approximately 350,000 cycles. The performance was as follows:
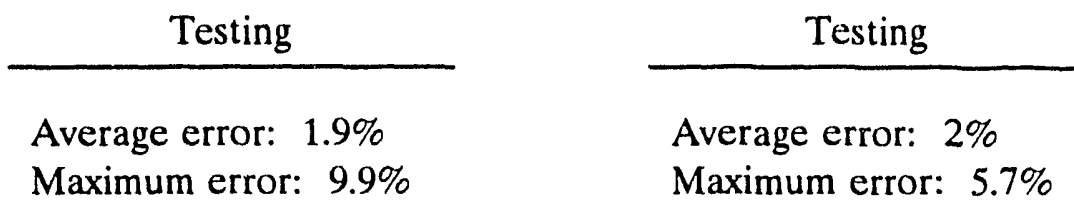

With this success, the amount of data on which the network should be trained was increased. The number of depth was increased from three to six and the number of days was increased from two to three. A single network was trained for approximately 120,000 cycles and had the following performance:

\begin{tabular}{|c|c|}
\hline Testing & Testing \\
\hline Average error: $1.9 \%$ & Average error: $4 \%$ \\
\hline Maximum error: $9.9 \%$ & Maximum error: $49 \%$ \\
\hline
\end{tabular}

The maximum error on testing data seemed too high. At this point, it was decided to train the network not on the moisture change but on the moisture values. Numerous networks on small subset, large, and extra large data sets were trained and tested. The small data set consisted of three depths, four distances, and 2 days. The best network had five hidden units and was trained for 63 cycles and had the following performance:

\begin{tabular}{ll}
\multicolumn{1}{c}{ Testing } & \multicolumn{1}{c}{ Testing } \\
Average error: $4 \%$ & Average error: $4.5 \%$ \\
Maximum error: $10 \%$ & Maximum error: $9 \%$
\end{tabular}

The large data set was composed of six depths, four distances, and 3 days. The best network had 10 hidden units and was trained for 28 cycles and had the following performance:

\begin{tabular}{|c|c|}
\hline Testing & Testing \\
\hline Average error: $4.3 \%$ & Avr rage error: $5 \%$ \\
\hline Maximum error: $9.8 \%$ & Maximum error: $11 \%$ \\
\hline
\end{tabular}

The extra large data set consisted of six depths, four distances, and 7 days. The best network had five hidden units and was trained for approximately 1,200,000 cycles and had the following performance:

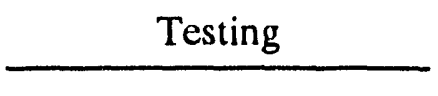

Average error: $.78 \%$

Maximum error: 5\%
Testing

Testing
Average error: $1.1 \%$
Maximum error: $6 \%$


These results are well within the requirements of site screening. However, the primary problem is that the resultirg neural network cannot be applied to other sites and still requires extensive sampling of the new site to adapt it to that site. 
Appendix C

Fully Recurrent Network Process Development 
C-2 


\section{Appendix C}

\section{Fully Recurrent Network Process Development}

A logical first step in the utilization of this approach is the construction of a network to model a small subset of the site data. This network consists of nine processing nodes each representing neighboring depths and distances from the injection well. This network is called $3 \times 3$ matrix. This network has two inputs that are the injection rate and initial moisture at each processing node as shown in Figure C-1. Each radius corresponds to a separate borehole, and each depth represents one of the 50 depths ranging from $300 \mathrm{~cm}$ to $1800 \mathrm{~cm}$ at $30 \mathrm{~cm}$ intervals. Every two nodes is connected with a weighted connection. The moisture predicted by each node represents the average moisture of the cell within which the node lies. Each cell measures $200 \mathrm{~cm}$ horizontally and $30 \mathrm{~cm}$ vertically. Moisture measurements from the third injection (14.4 days to 21 days) were used to train the networks. This translates into 132 moisture measurements per processing node per training cycle. The results will be shown only for the processing node of a distance of $100 \mathrm{~cm}$ and a depth of $540 \mathrm{~cm}$. Various networks with different total RMS error requirements, different hidden units were trained and tested. The best results were obtained after 1,000 training cycles to a total RMS error of $0.5 \%$ with no hidden units. Figure C-2 shows the network's performance in free recall mode. In this mode, the network is provided with initial moisture measurements at each cell and required to predict the remaining moisture values through time. As shown in Figure C-2, the network performance is satisfactory. Figure C-3 shows the networks performance in forced recall mode. In this mode the network is provided with the actual moisture measurements at each time interval, and it is required to predict the moisture value at the next time interval. Therefore, its prediction must be better than the free recall mode, which is the case.

Figure C-4 shows the network generalization capability. In this figure actual injection rates and initial moisture $(t=0)$ are the input for the network. This figure shows that moisture spikes should have been recorded during experiments on approximately day 35 and day 65 . One explanation could be that the measured values were recorded poorly, or a faulty device was utilized. For this reason, it was decided that the networks will be analyzed only for the first five injections. This network was not able to completely generalize to different initial moisture conditions or different injection rates. It was then decided to add the initial moisture values before each injection for both training and testing. Therefore, in addition to the magnitude of the current injection rate, this network also receives the moisture values of all of the cells as was measured immediately before the injection takes place. These moisture values represent the current base-line conditions before the current injection. Various networks were designed and trained. The best network was trained on first three injections for 165 cycles, nine hidden units and a RMS value of $0.72 \%$. Figures C-5 through C-10 demonstrate this network's prediction ability on both individual injections as well as running predictions.

The results indicate that this approach is somewhat better than the previous approach by comparing Figures C-4 and C-10. On average, the predicted peaks and initial moistures are closer to the measured values.

These results indicate that the fully recurrent network approach has the potential to predict moisture levels of various injections, once the network is trained on data related to a single injection. Based on these results, torsls are being developed that could predict the moisture levels at certain 
depths from moisture values measured at more shallow depths. Sticking to our nine processing nodes, we would like to know whether we can predict the moisture values at six lower boreholes given the moisture values at three upper boreholes. This format is called three- to six-setup. Various networks with total RMS error requirements of $0.5 \%$ and different number of hidden units were trained and tested. The best results obtained after 1550 training cycles to a total RMS error of 0.005 with six hidden units. Figure $\mathrm{C}-11$ shows the network's performance in free recall mode. This network has been trained on third injection. Figure $\mathrm{C}-12$ shows the network generalization capability to two other injections which look satisfactory. It was then felt that the two upper boreholes away from the injection site might not be so effective in predicting the moisture levels of lower boreholes since their moisture values would not r'ıange much with time. The new format is called one- to six-setup. Different networks with different number of hidden units were trained and tested. The best results obtained after 2,165 training cycles to a total RMS error of 0.005 with three hidden units. Figure C-13 shows the network's performance in free recall mode. This network has been trained on third injection. Figure C-14 shows the network generalization capability to other four injections that seem to be satisfactory.

We then used the network to extend its generalization capability to other locations (i.e., higher depth). We also incorporated the soil hydraulic conductivity parameter as an extra parameter to see if the network can predict the moisture level at other locations more accurately.

\section{Generalization Capability with Respect to Depth}

This network was tested on two other depths, 810 and $840 \mathrm{~cm}$. Figures $\mathrm{C}-15$ through C-20 show the results for radial distances of 1,3 , and $5 \mathrm{~m}$. The results were not satisfactory. We then began a series of tests to see if we could find any correlation between the learning at different depths and the connecting weights in the network. The training was performed for three networks starting at depths of 540,780 , and $1080 \mathrm{~cm}$. No correlation was observed between the connecting weights. Even the correlation between the connecting weights was weak when the three networks to be trained overlapped. In this situation, the distance between the successive networks was $60 \mathrm{~cm}$. This led us to believe that soil properties could be important parameters if we want to generalize the network with respect to other locations.

\section{Networks with Soil Properties}

Saturated hydraulic conductivity constant of soil was added to the network input parameters. We had to make sure that the training network saw multiple soil types. A network consisted of six different depths $(540,600,660,750,810$, and $960 \mathrm{~cm})$ was trained. Only injection No. 3 was used for the training. In this exercise, we let the network see only real data rather than 0.05 day interpolated data. This network was tested on other depths and the results didn't show a good generalization. It was then decided to use soil type $(1,2,3,4)$ rather than saturated hydraulic conductivity constant as an input to the network. This input was given to network as binary so that each depth would have four bits to represent the soil type. One would be on and the other three would be off for each depth. This meant 12 new inputs to replace the three soil conductivity constant inputs. This network was trained for various hidden units and the best results were obtained for three hidden units. The generalization results with respect to iime (from one injection to five injections), with respect to depth (from 570 to $990 \mathrm{~cm}$ ), with respect to radial distance (from 1 to $5 \mathrm{~m}$ ) and with respect to radial position (from Radial A to Radial B) are shown in Figures C-21 
through C-31. Figures C-21 through C-23 predict the moisture content as function of radial distance for five injections. Figures C-24 through C-29 predict the moisture content for two other depths as a function of radial distance. Figure C-30 predicts the moisture at another depth at radial distance of $1 \mathrm{~m}$. Figure C-31 predicts the moisture at another radial position (Radial B). It is important to note that the network was not trained by any data from Radial B locations. The predictions shown in Figures C-21 through C-31 indicate that the network generalization capability to other injections, depths, radial positions, and radial distances seem to be satisfactory. 


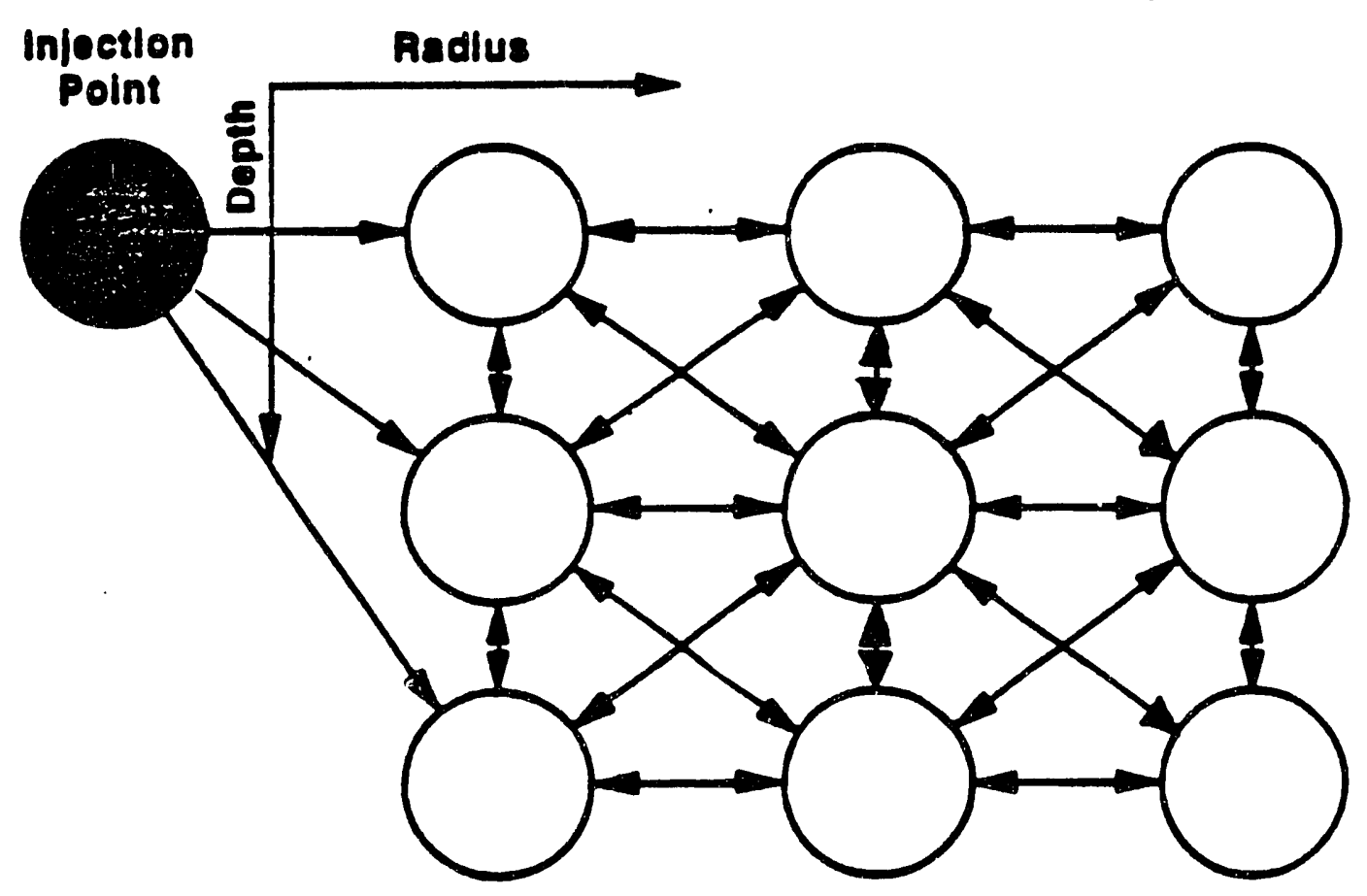

Figure C-1. Fully recurrent network. 


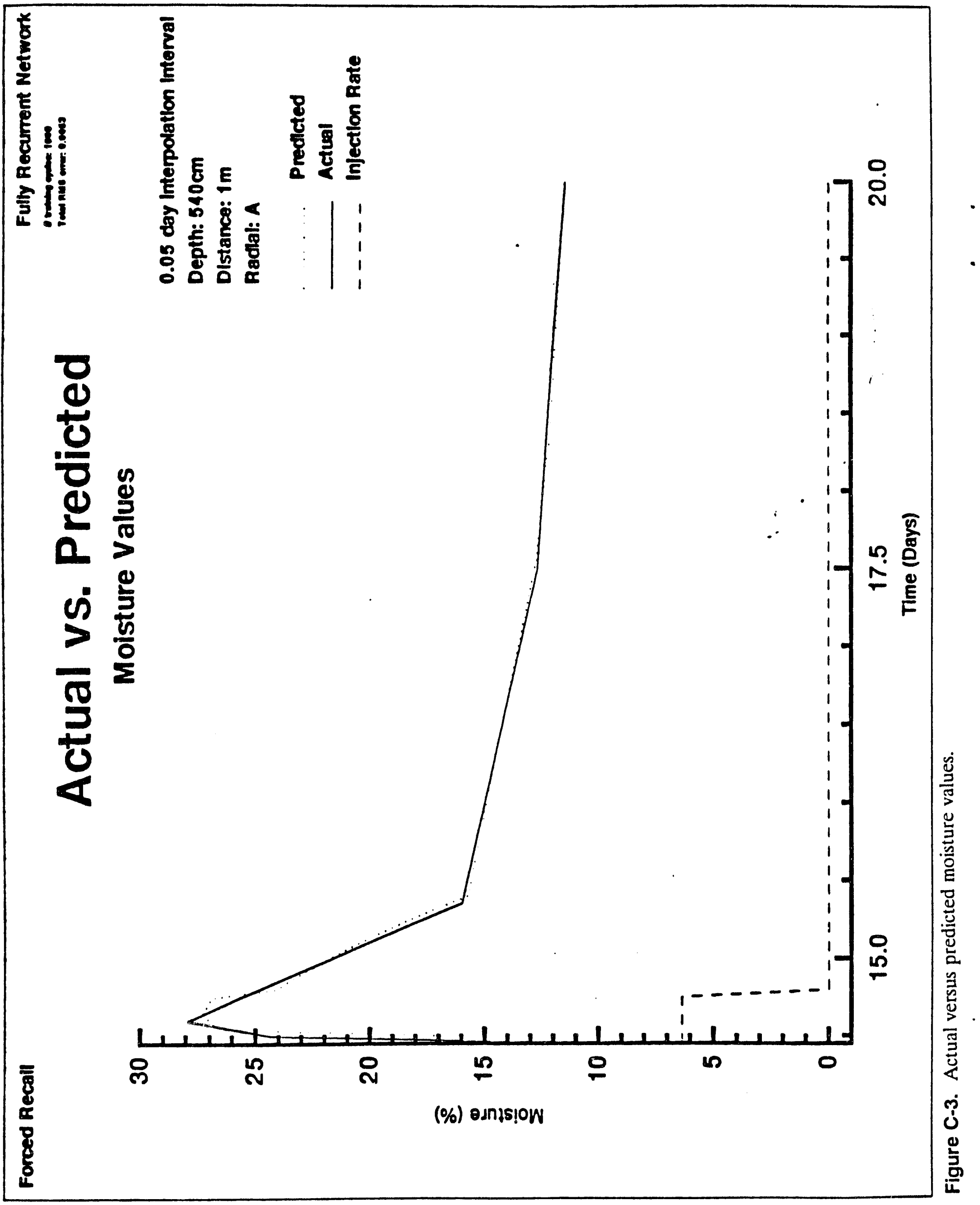




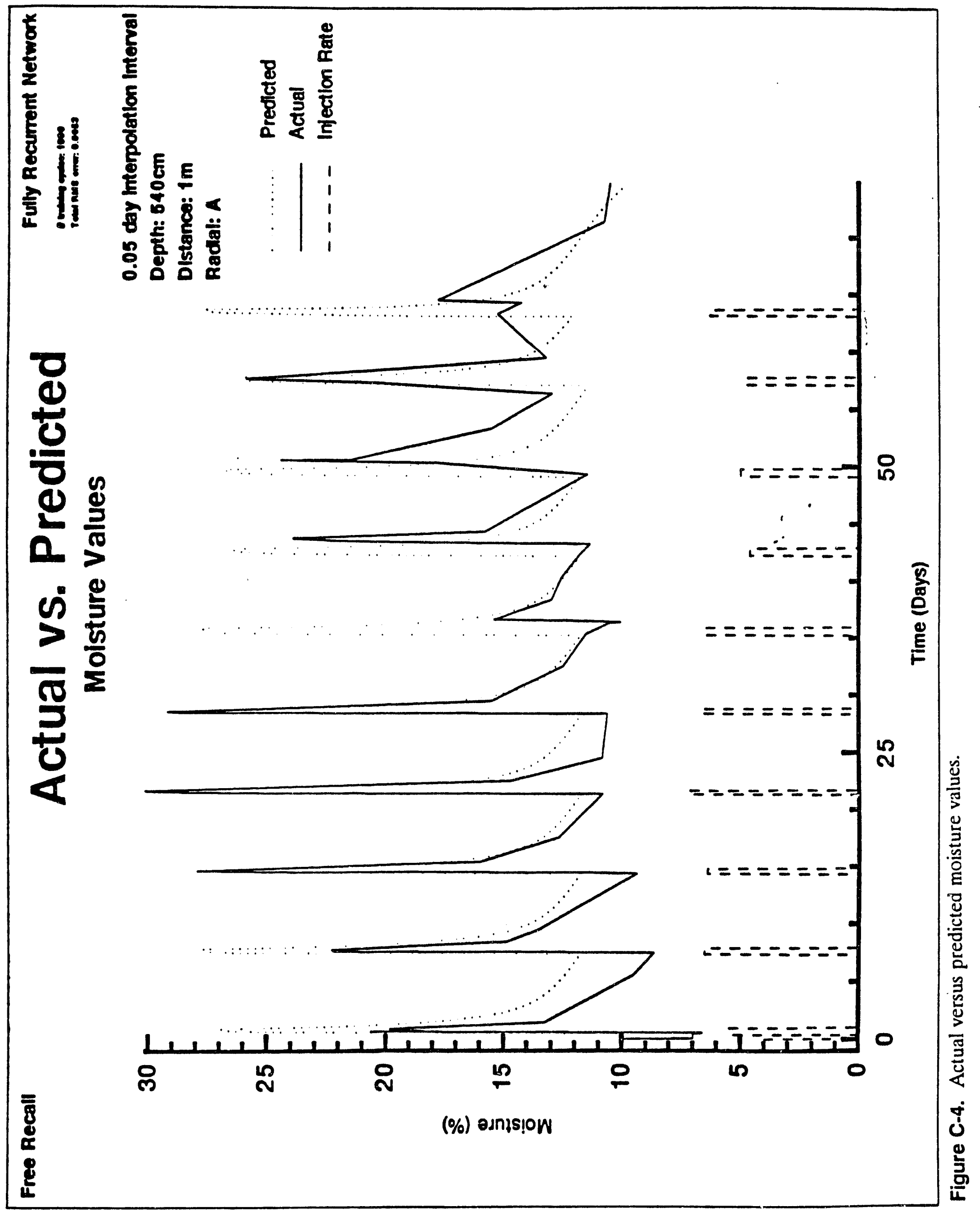




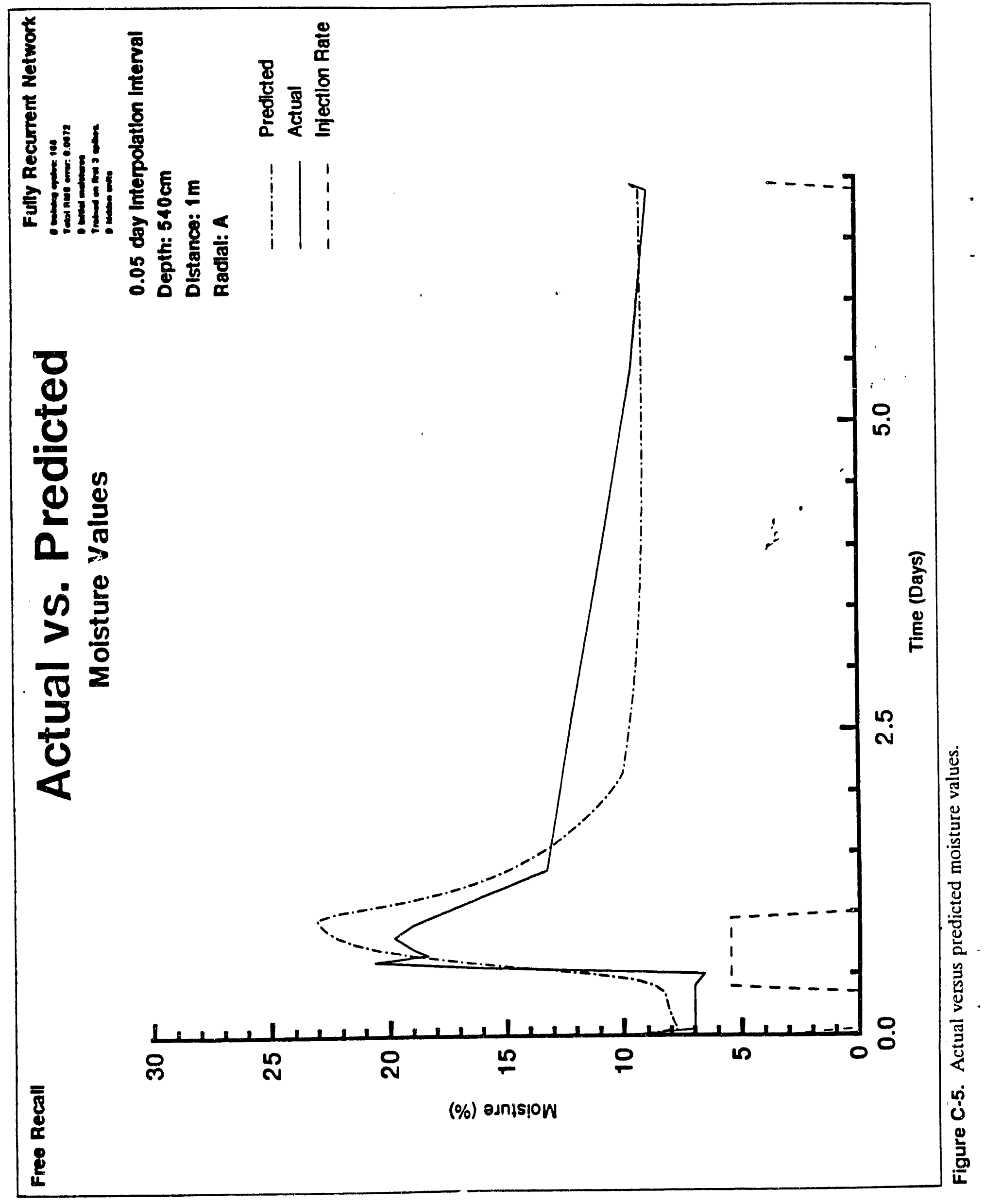

C-10 


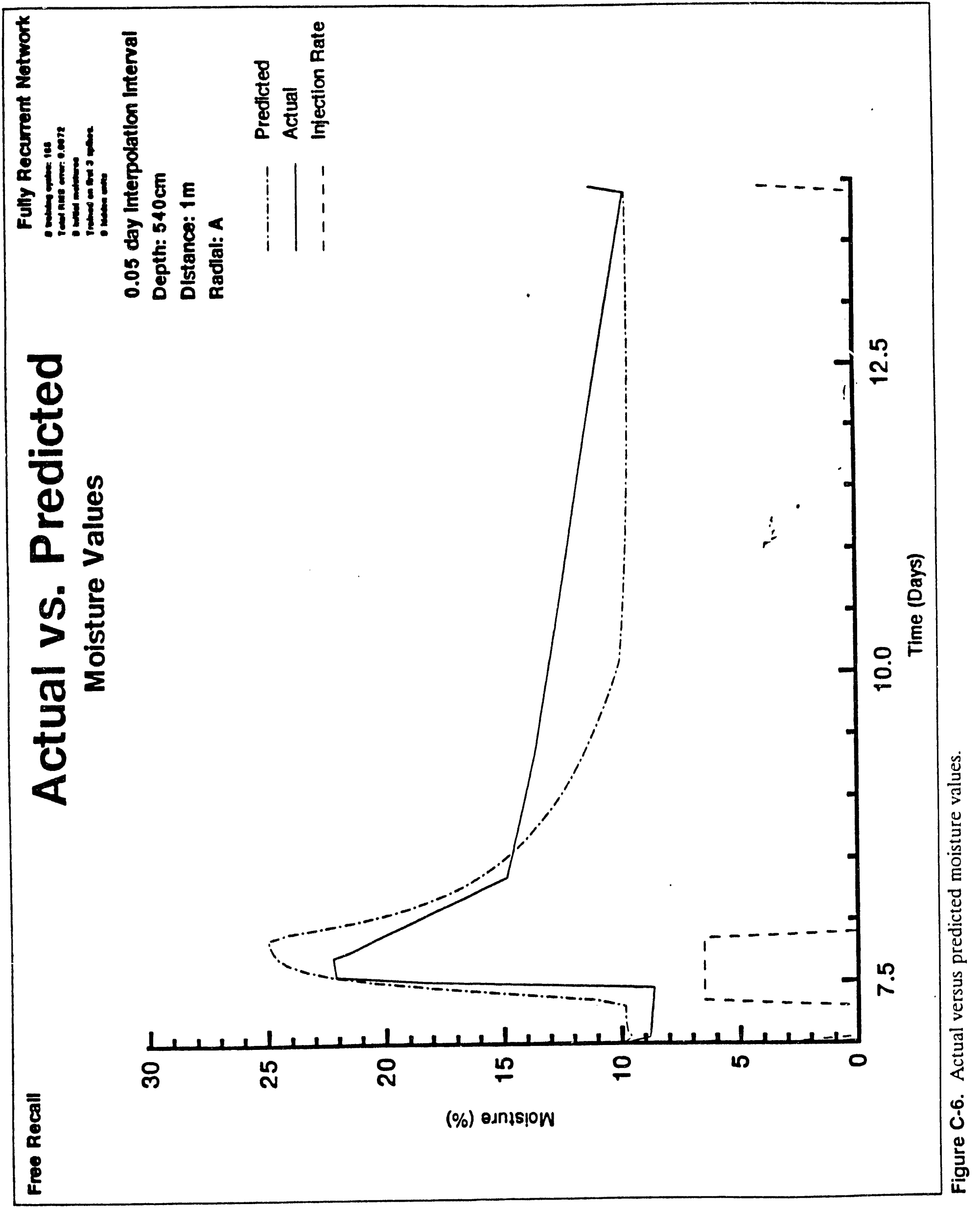

C-11 


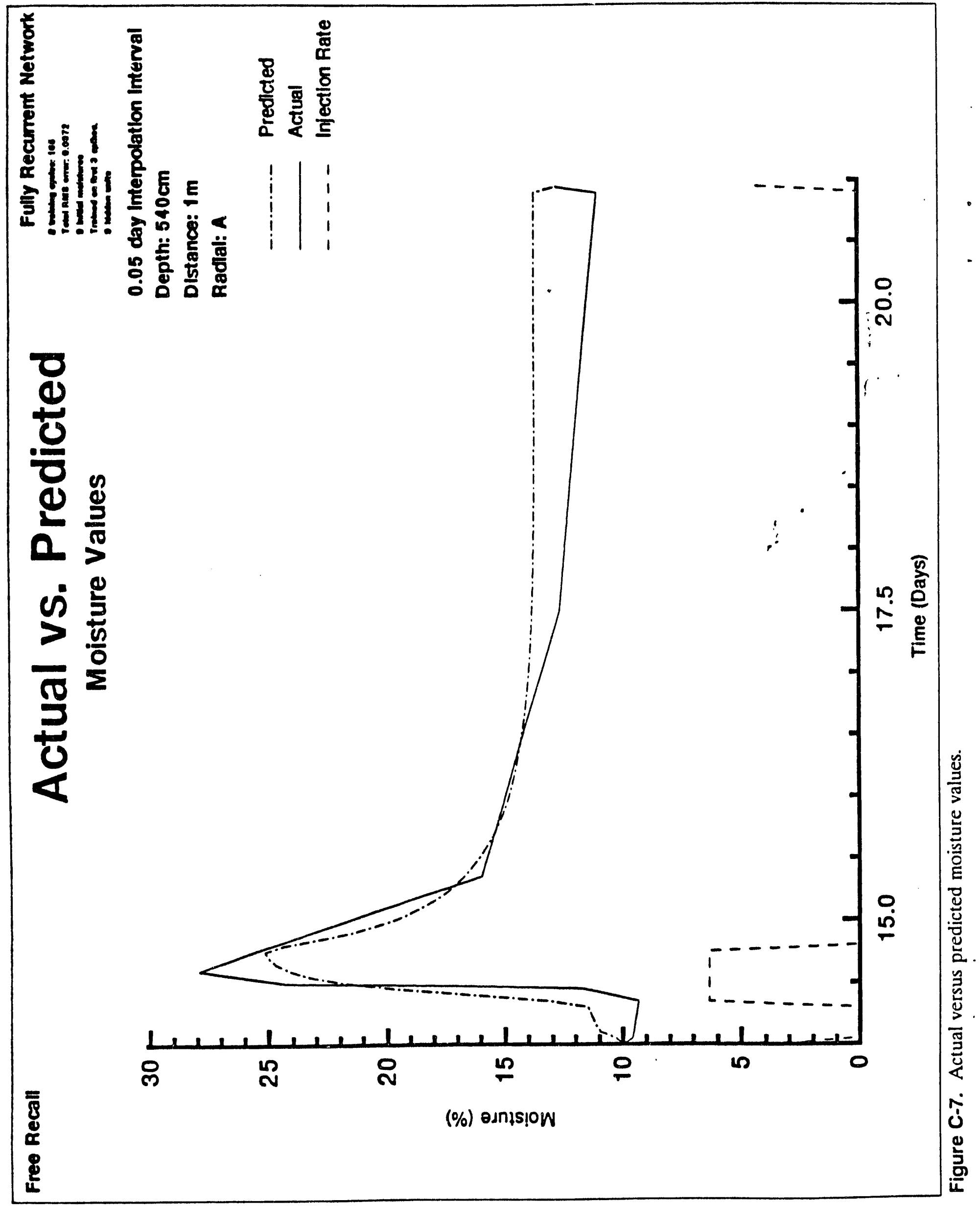

C-12 


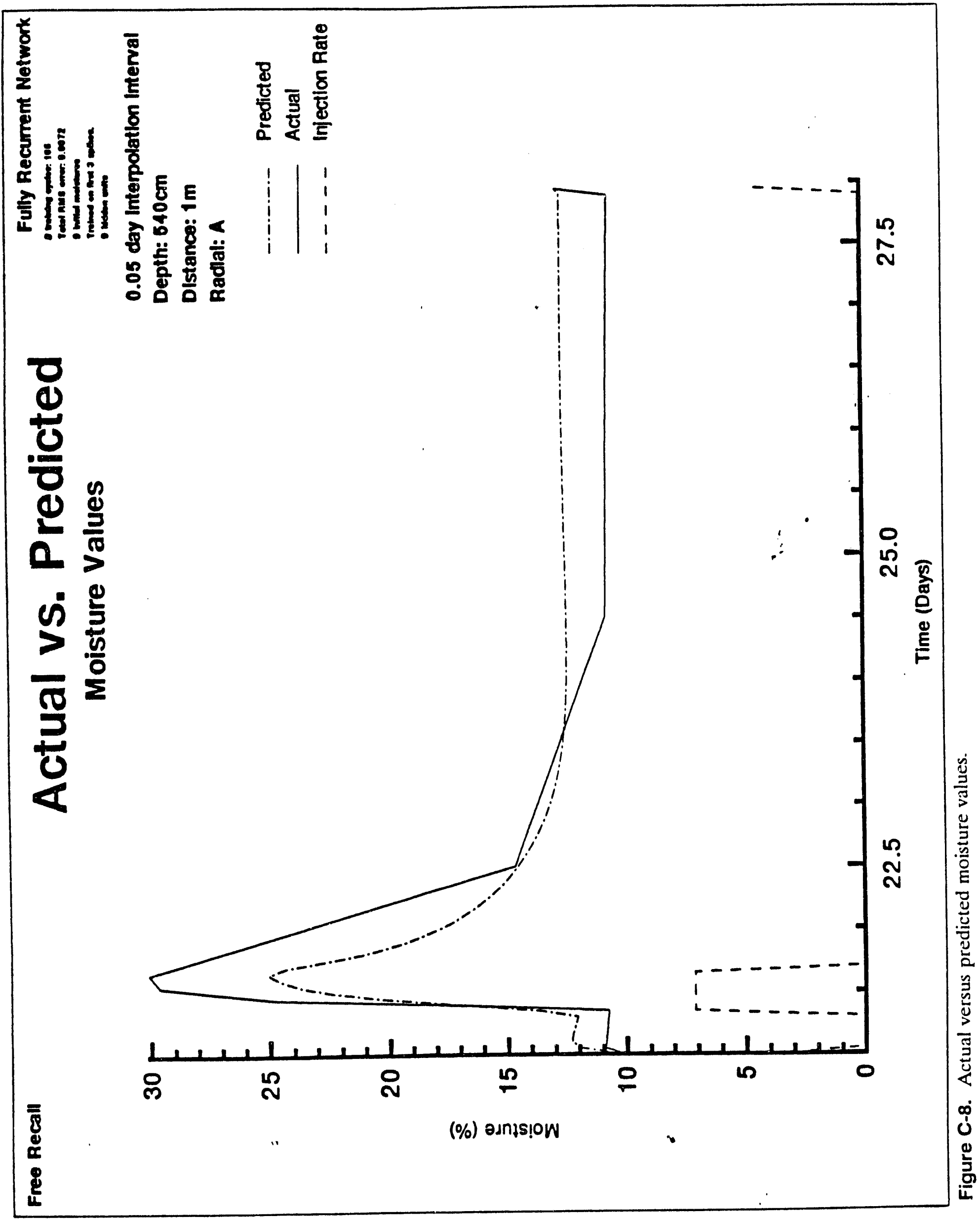

C-13 


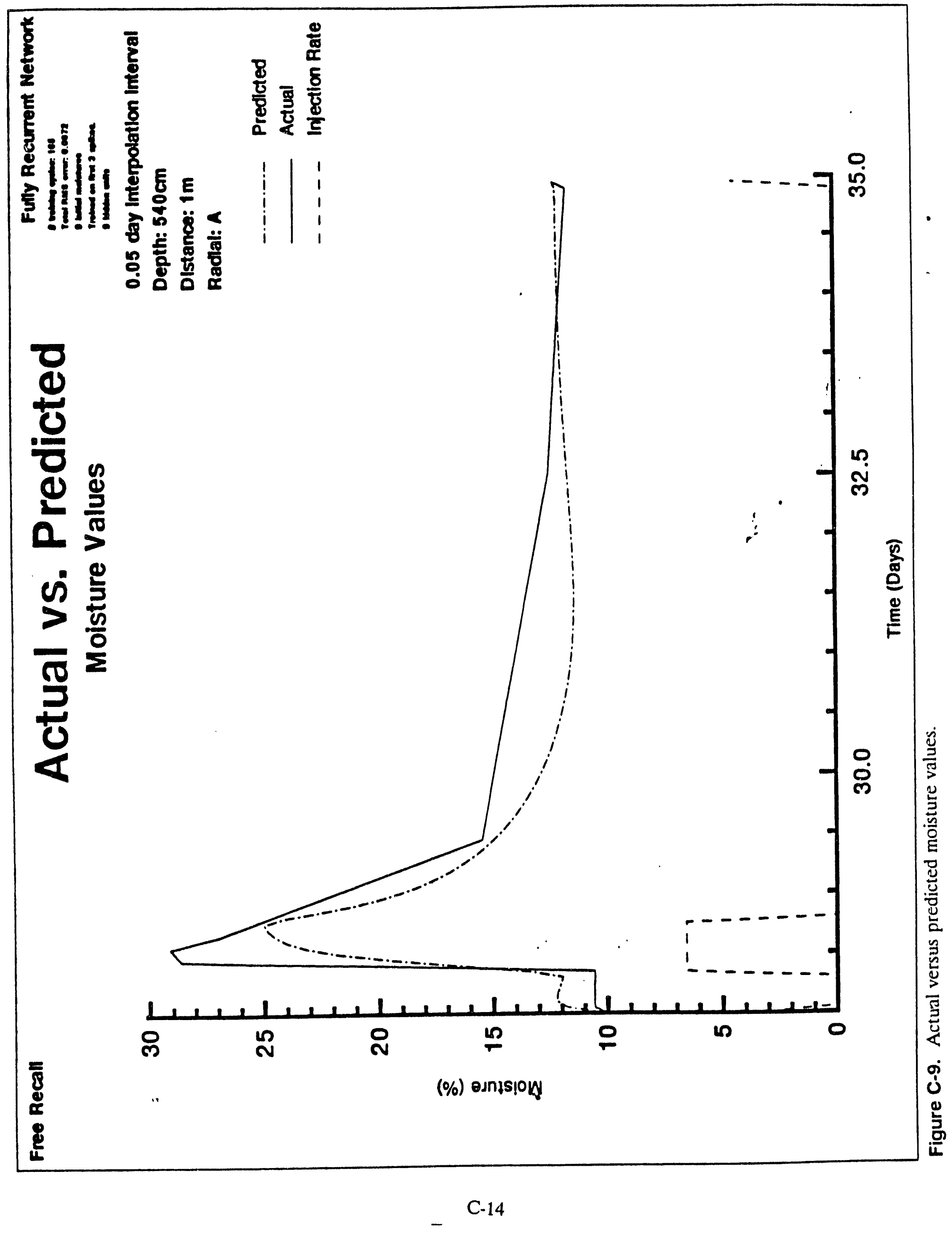




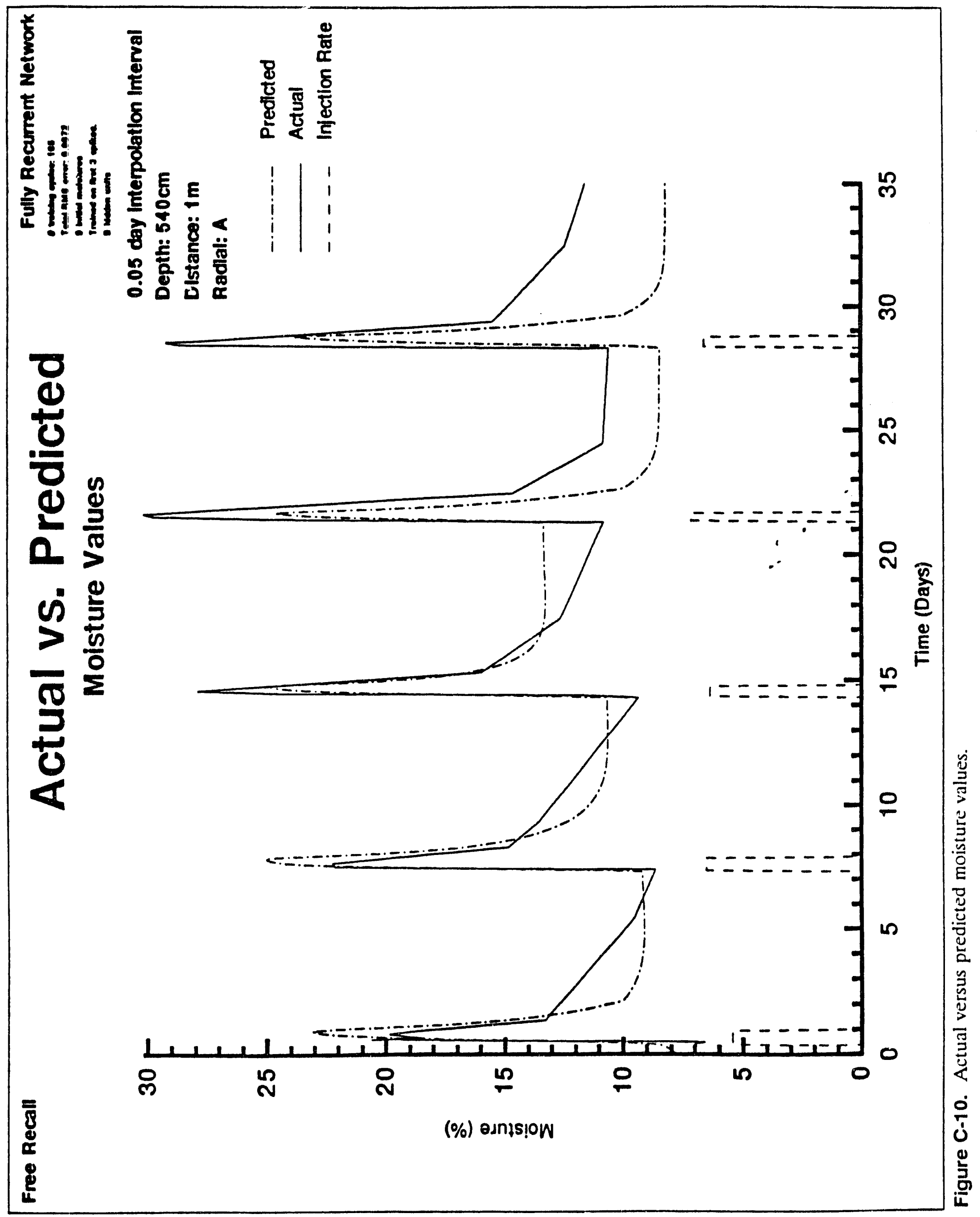

C- 15 


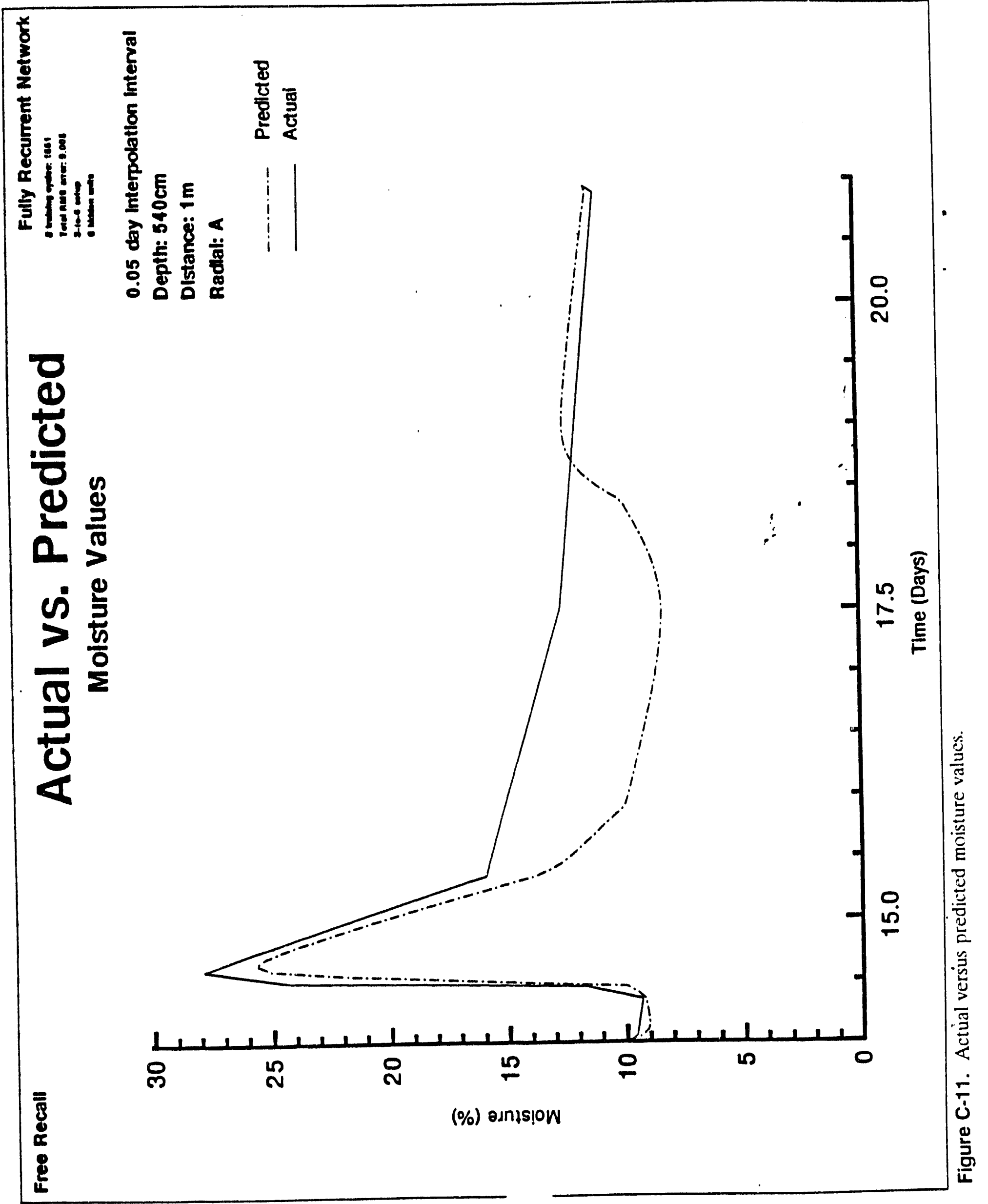

C-16 


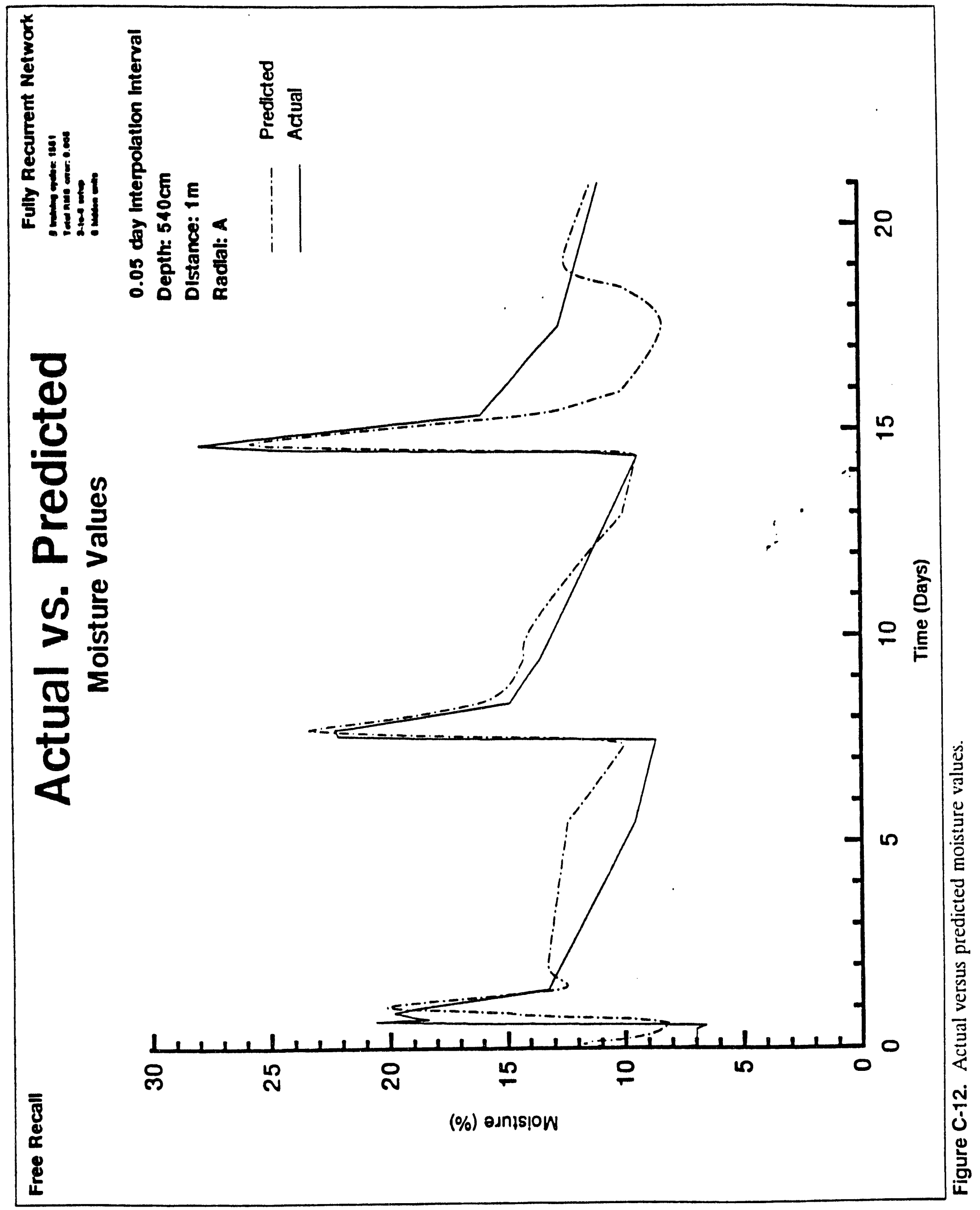

C-17 


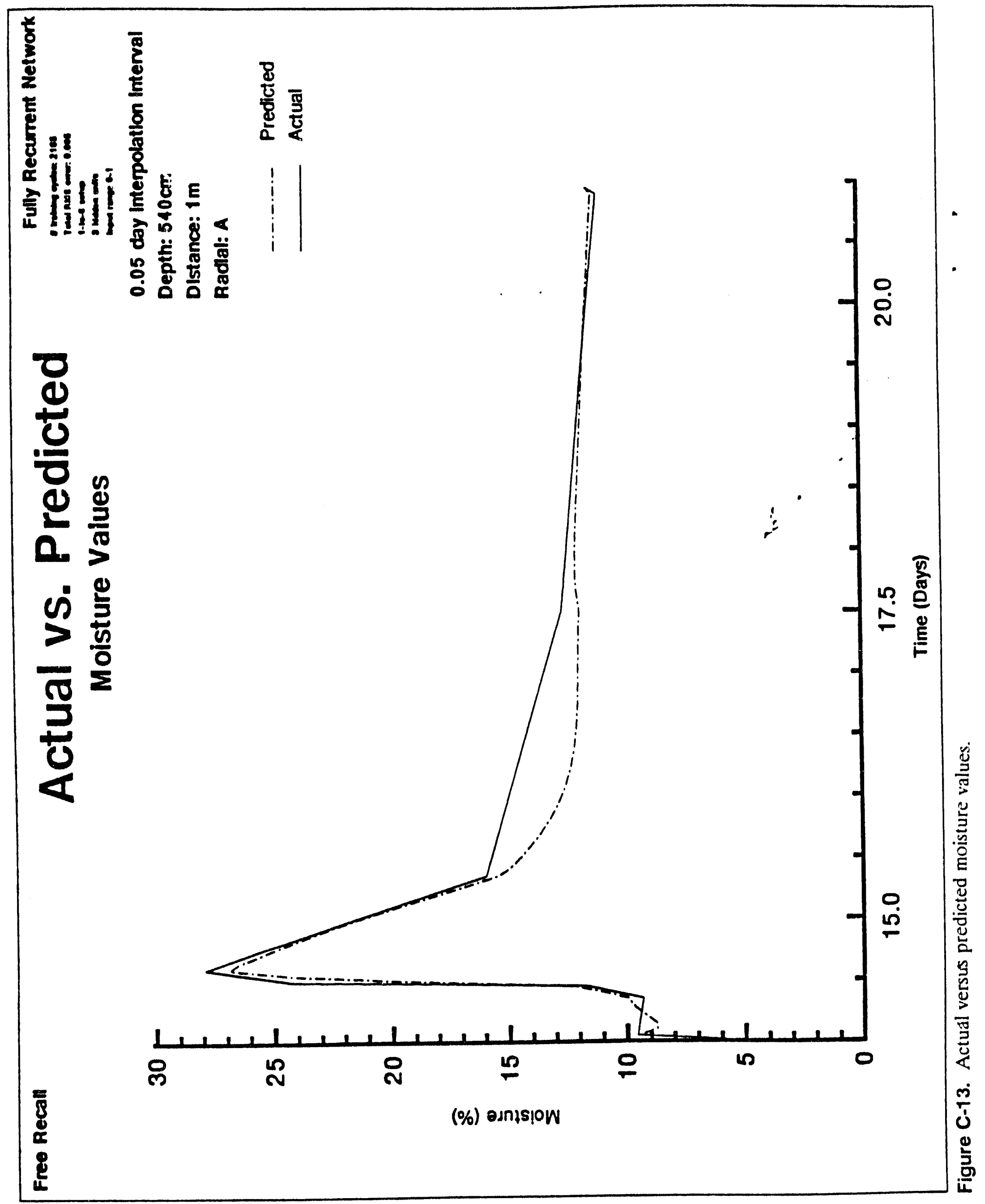

C-18 


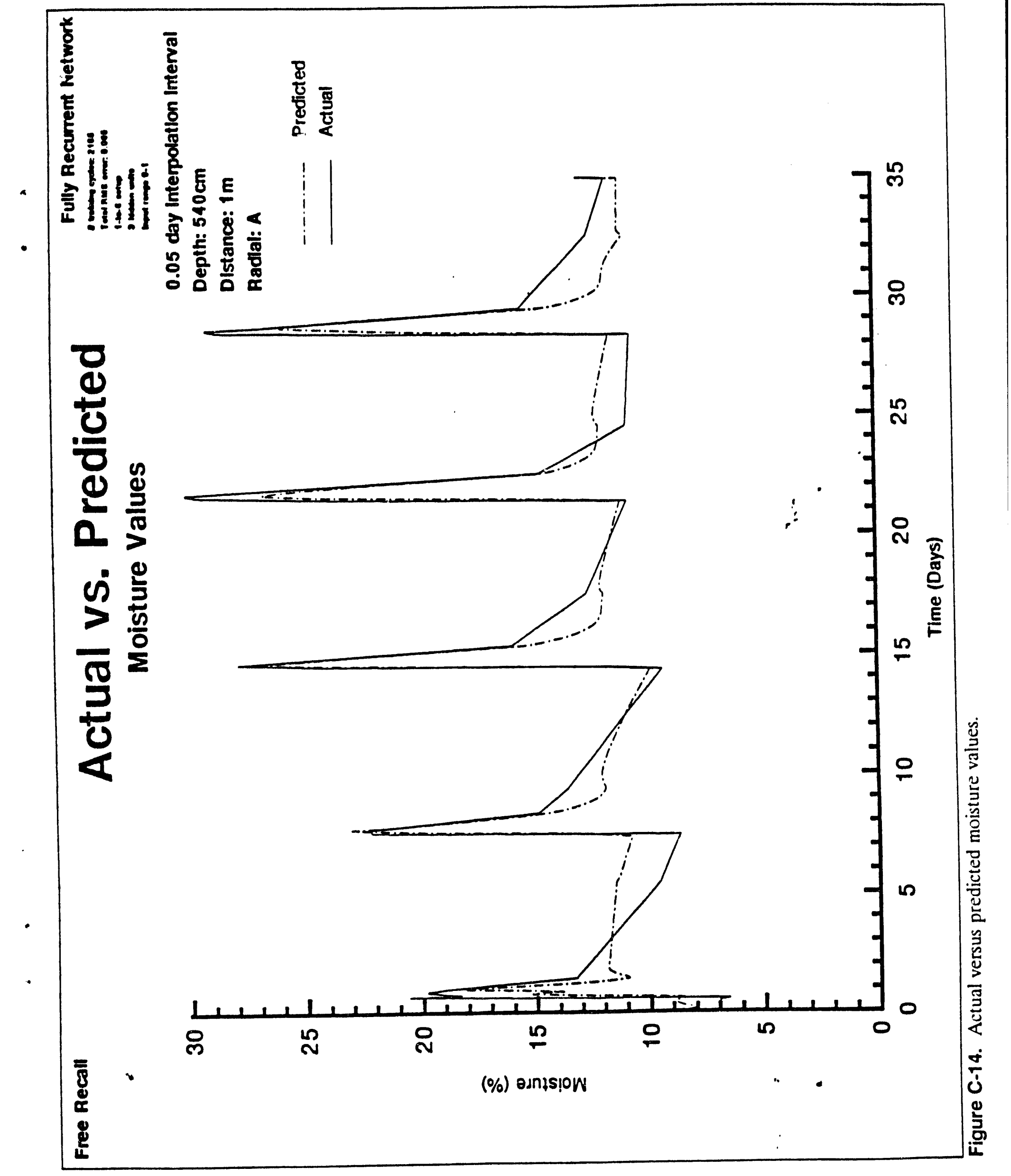

C-19 


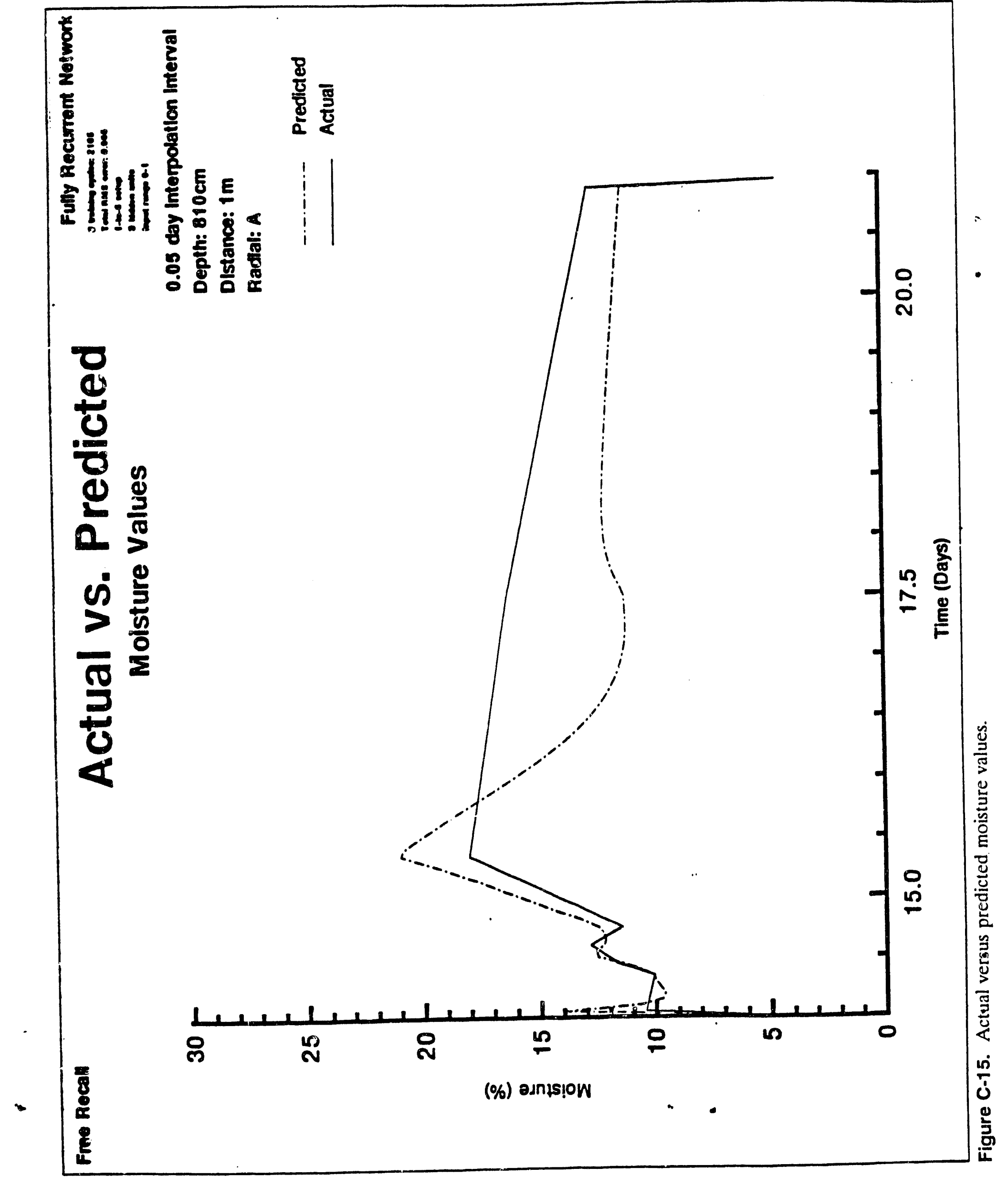




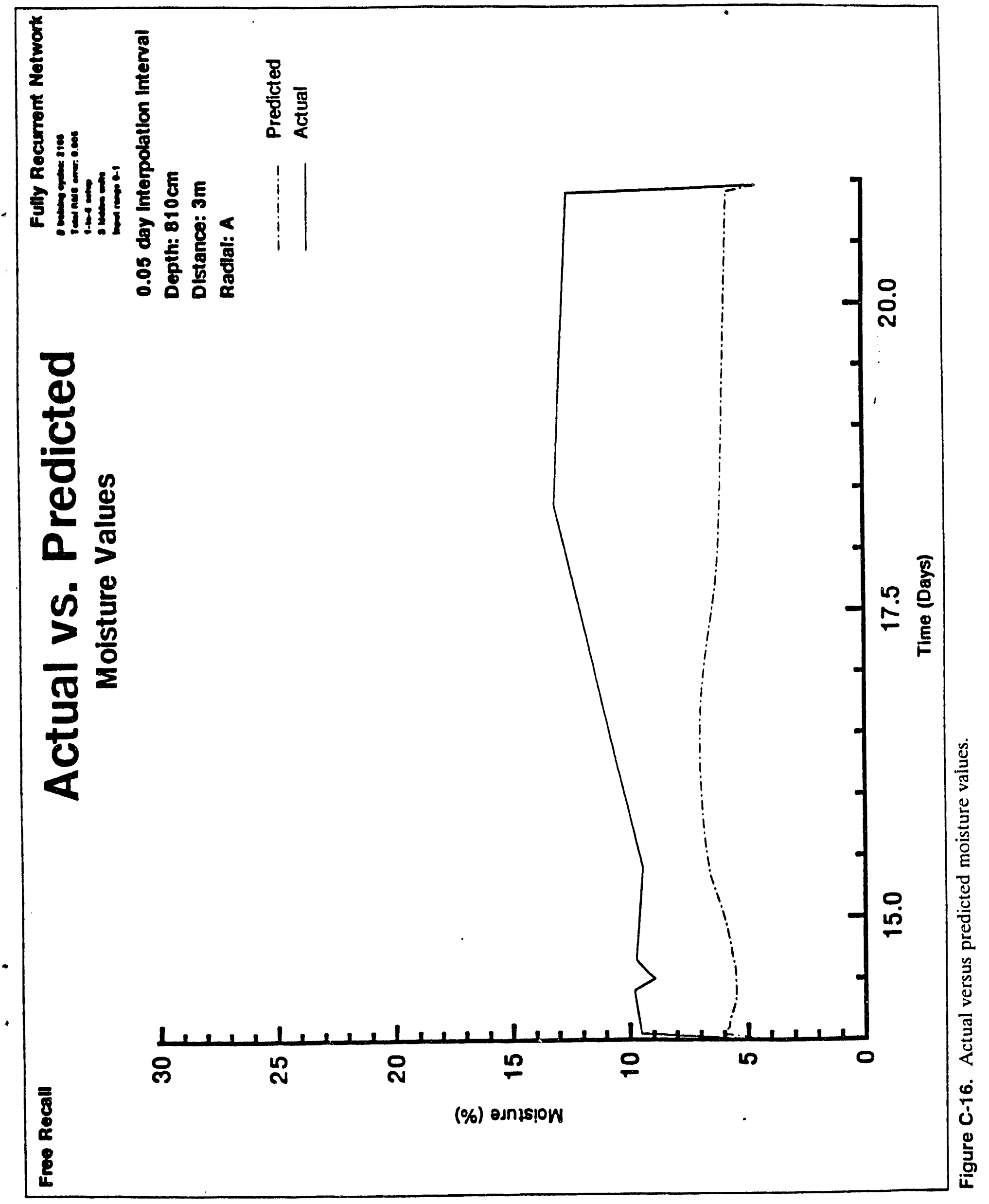

C-21 


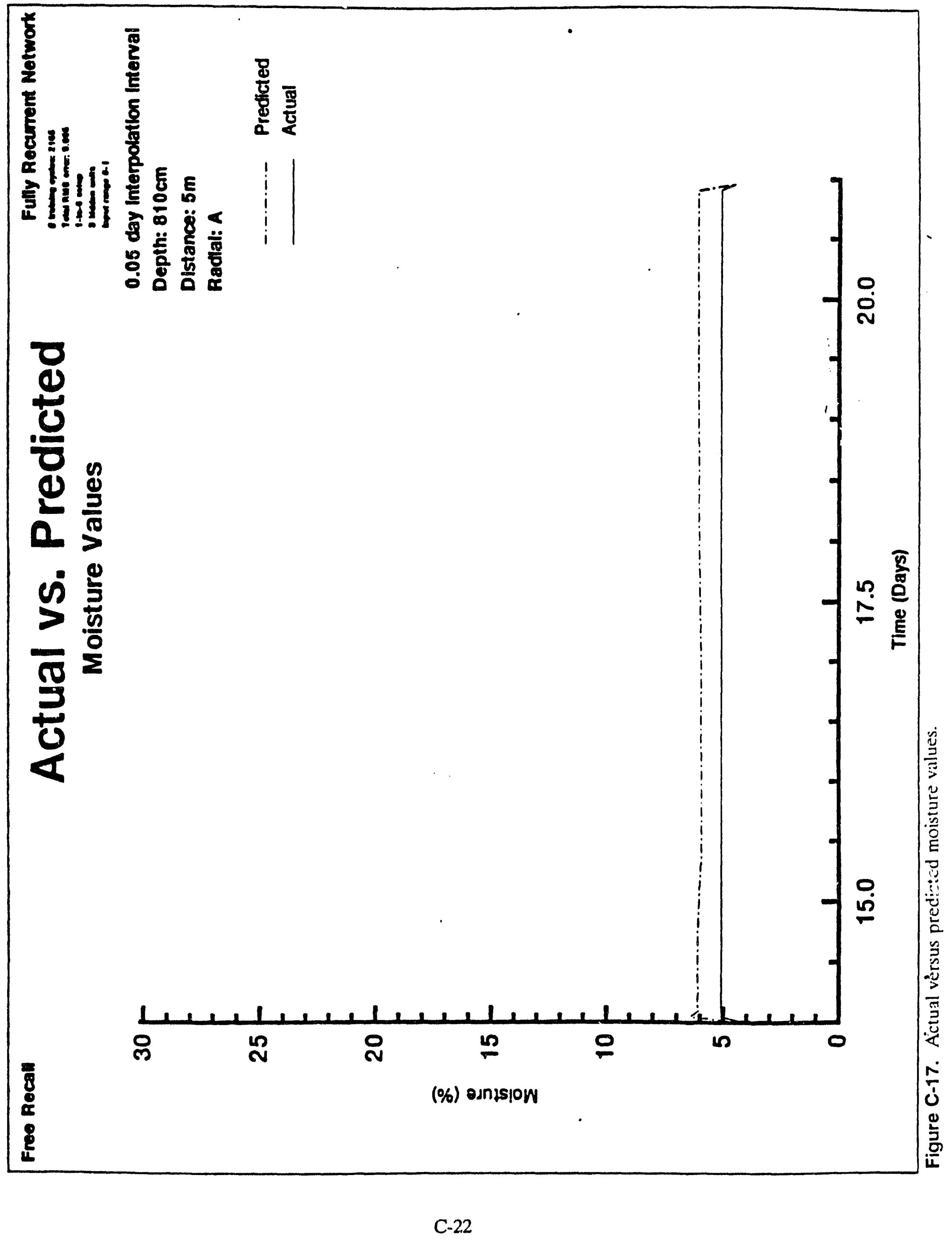




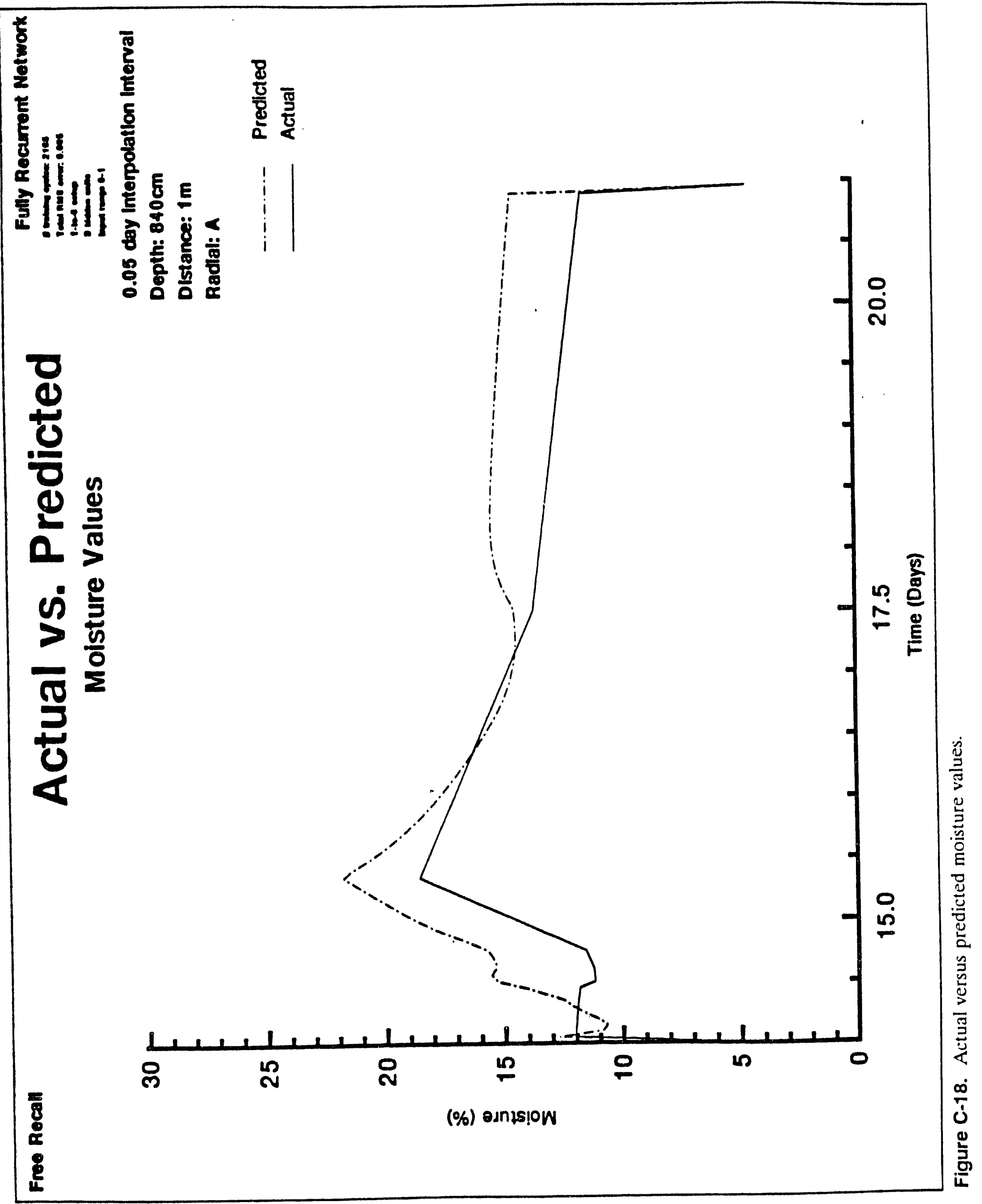




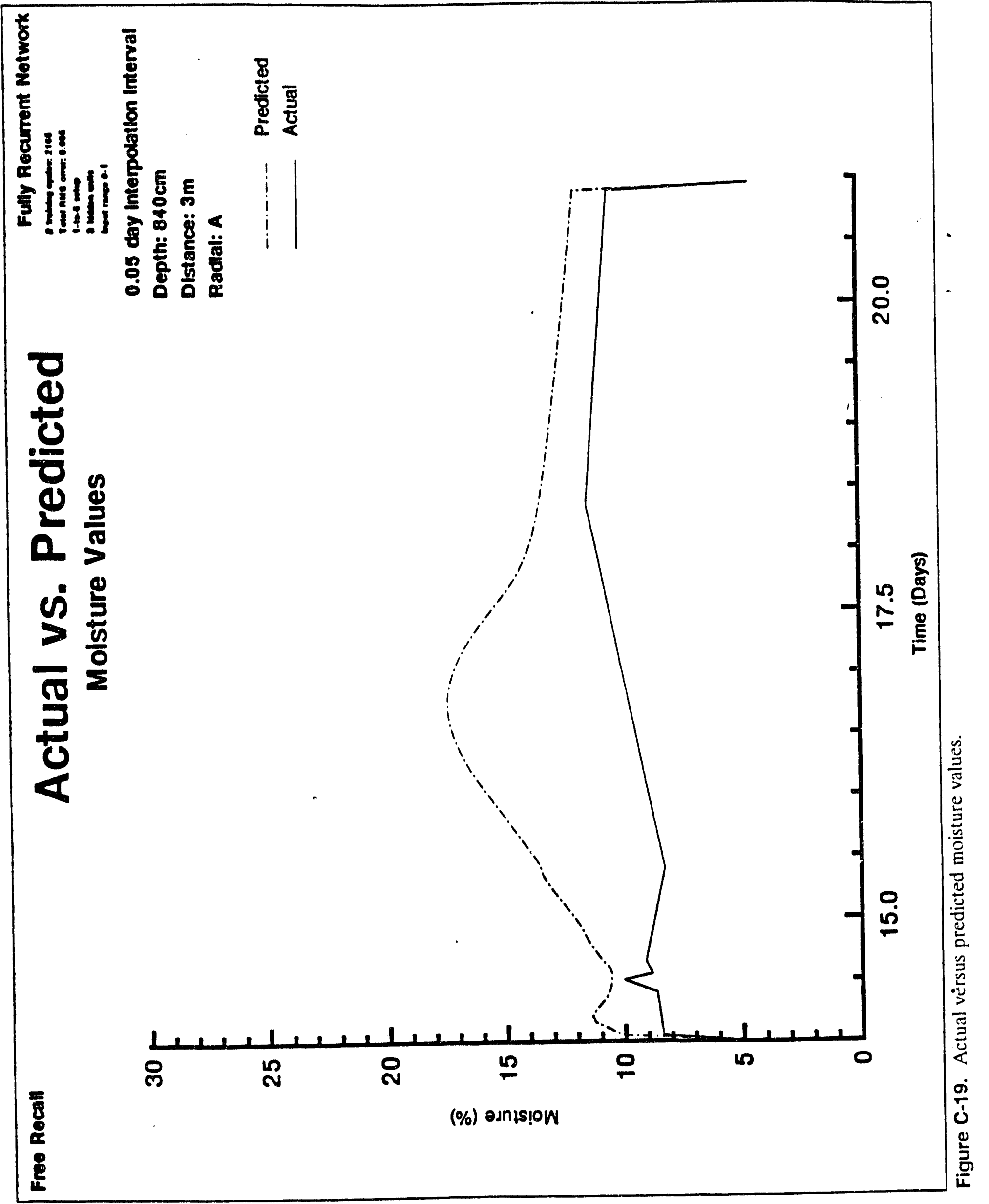




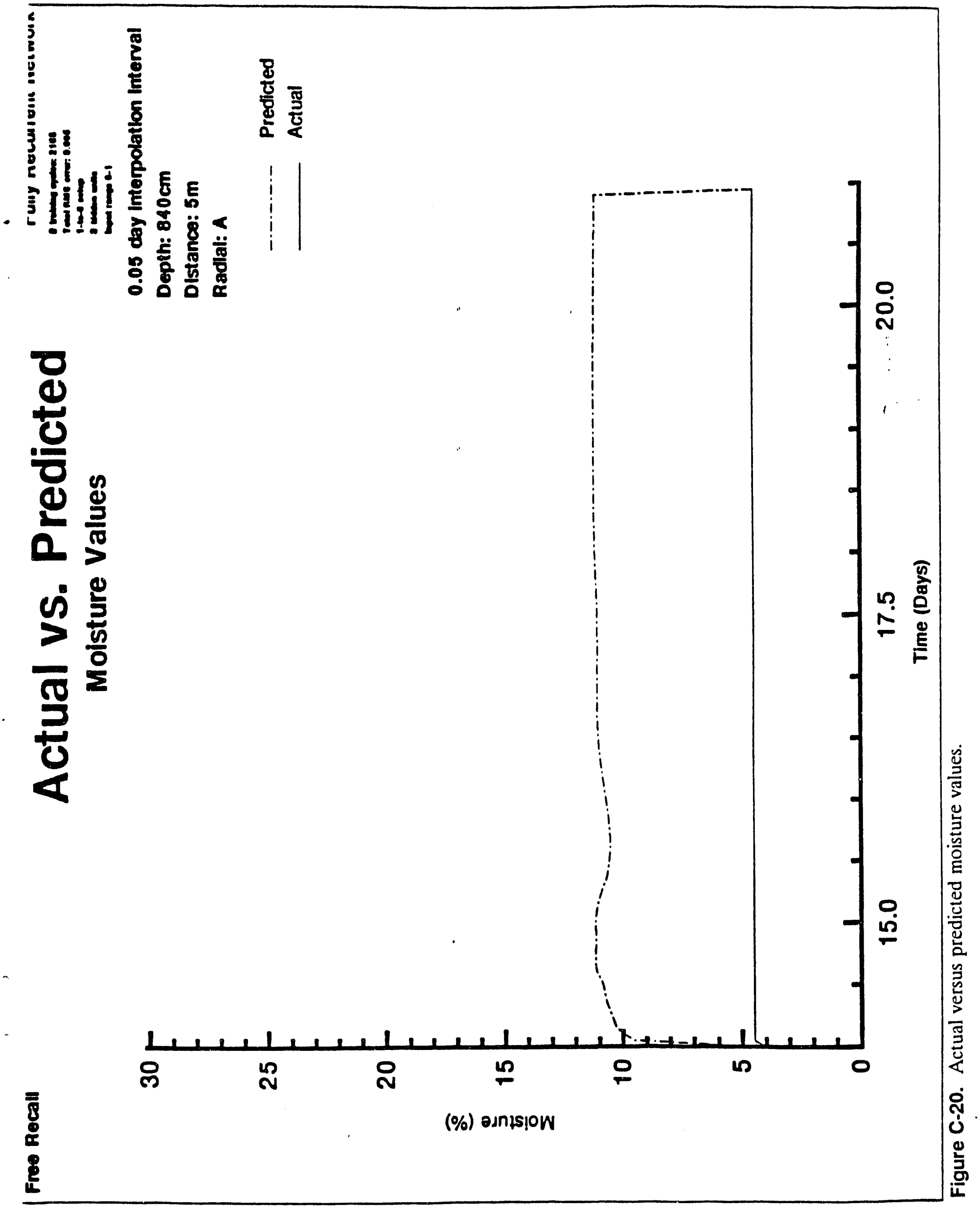




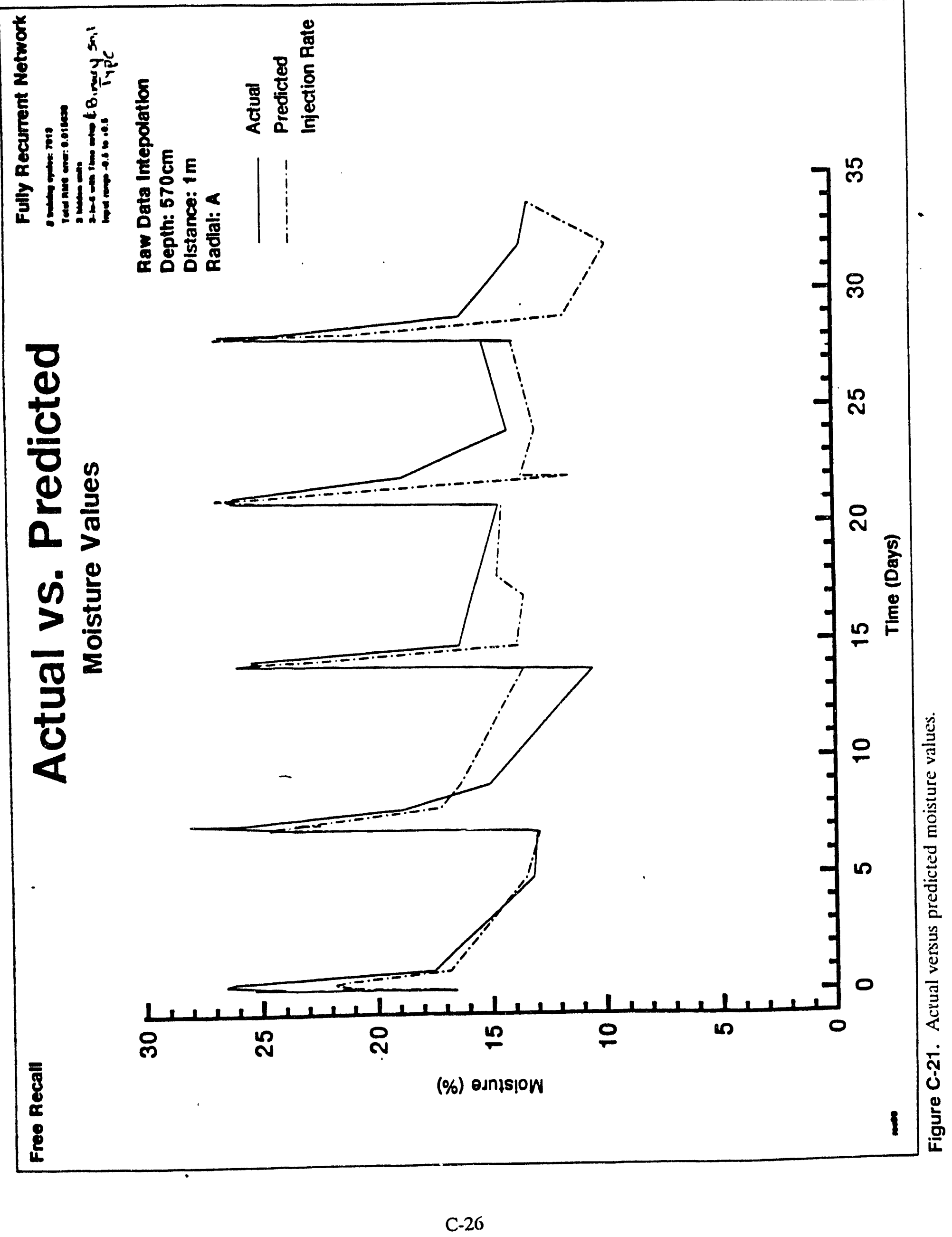




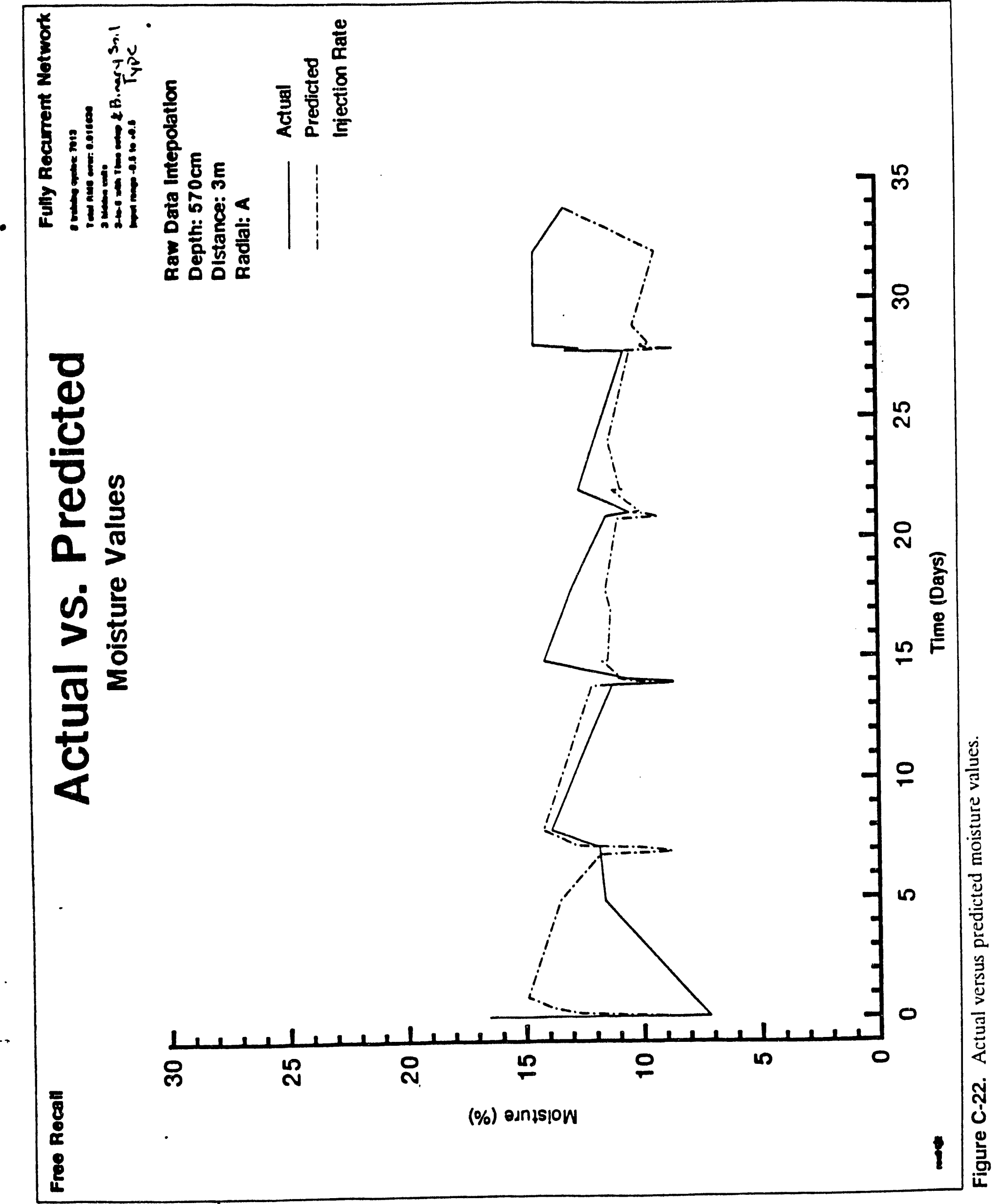




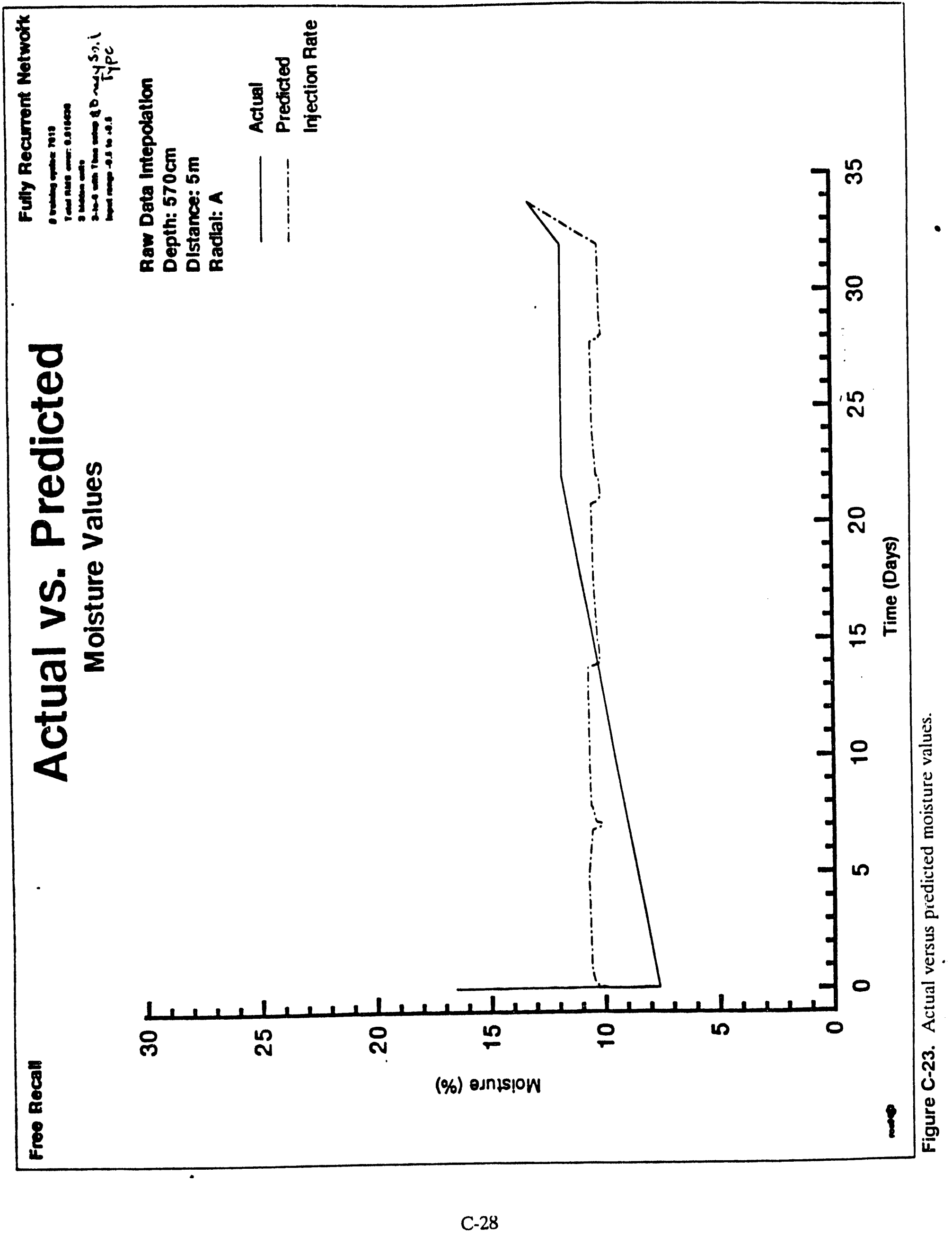




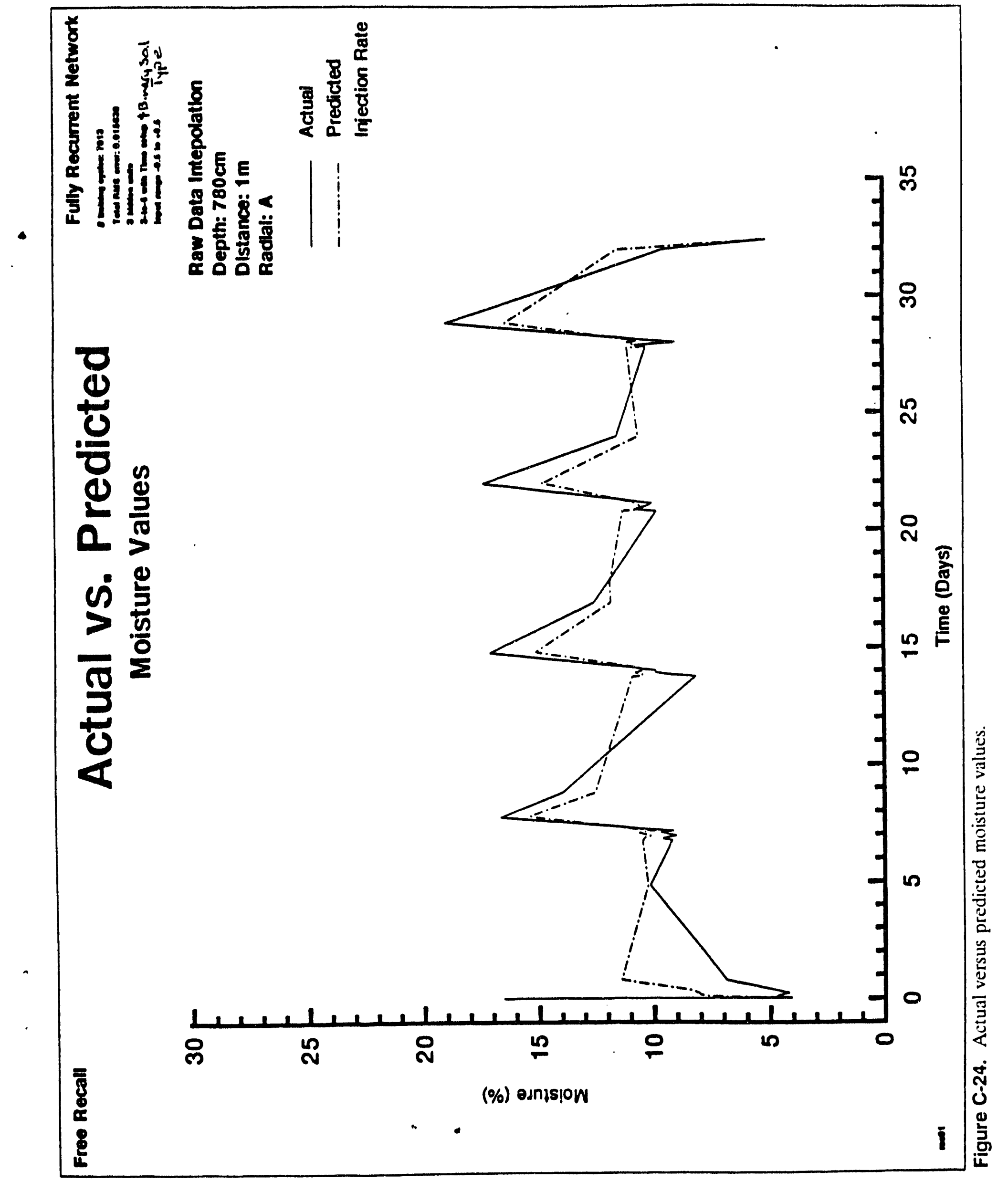




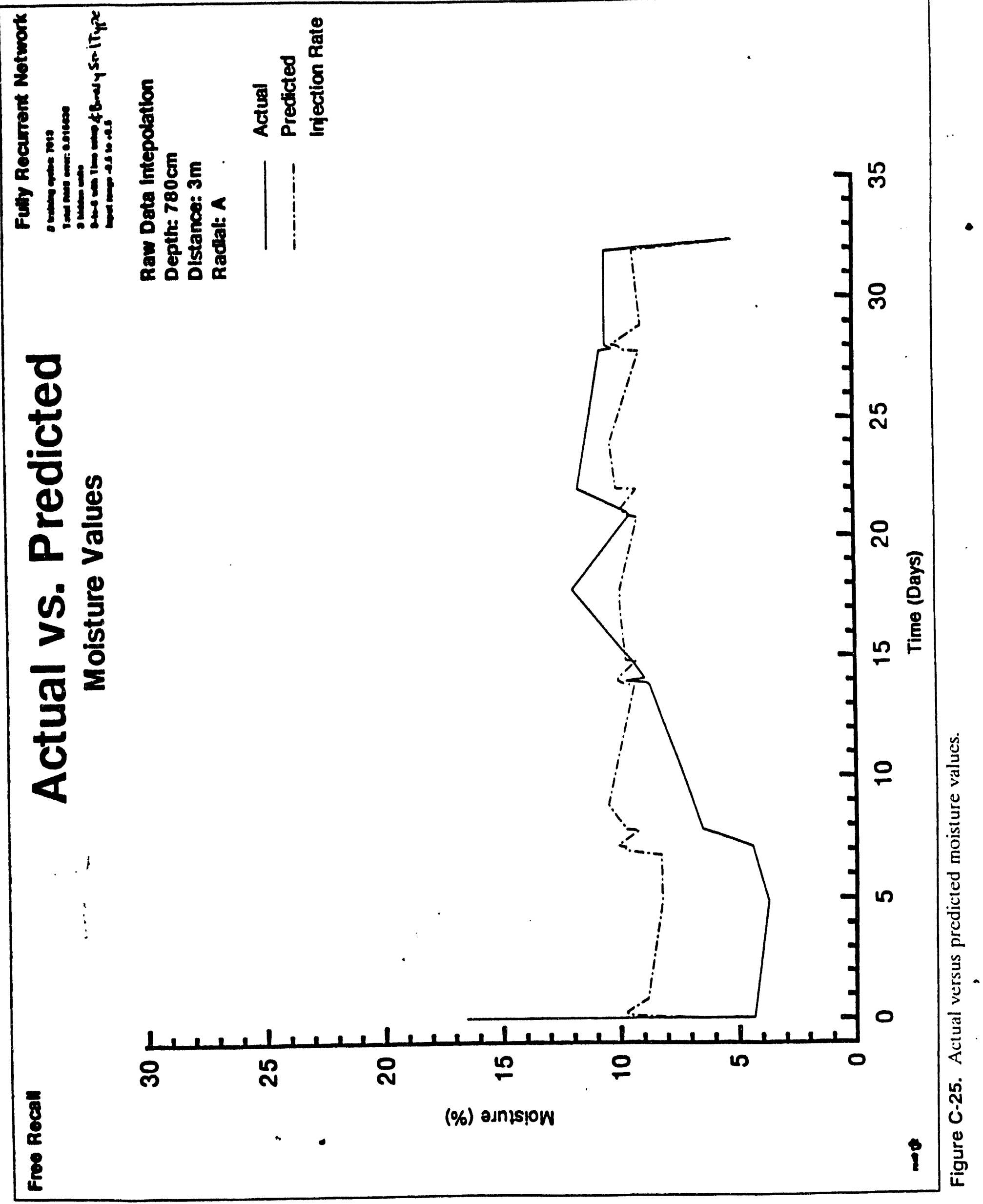




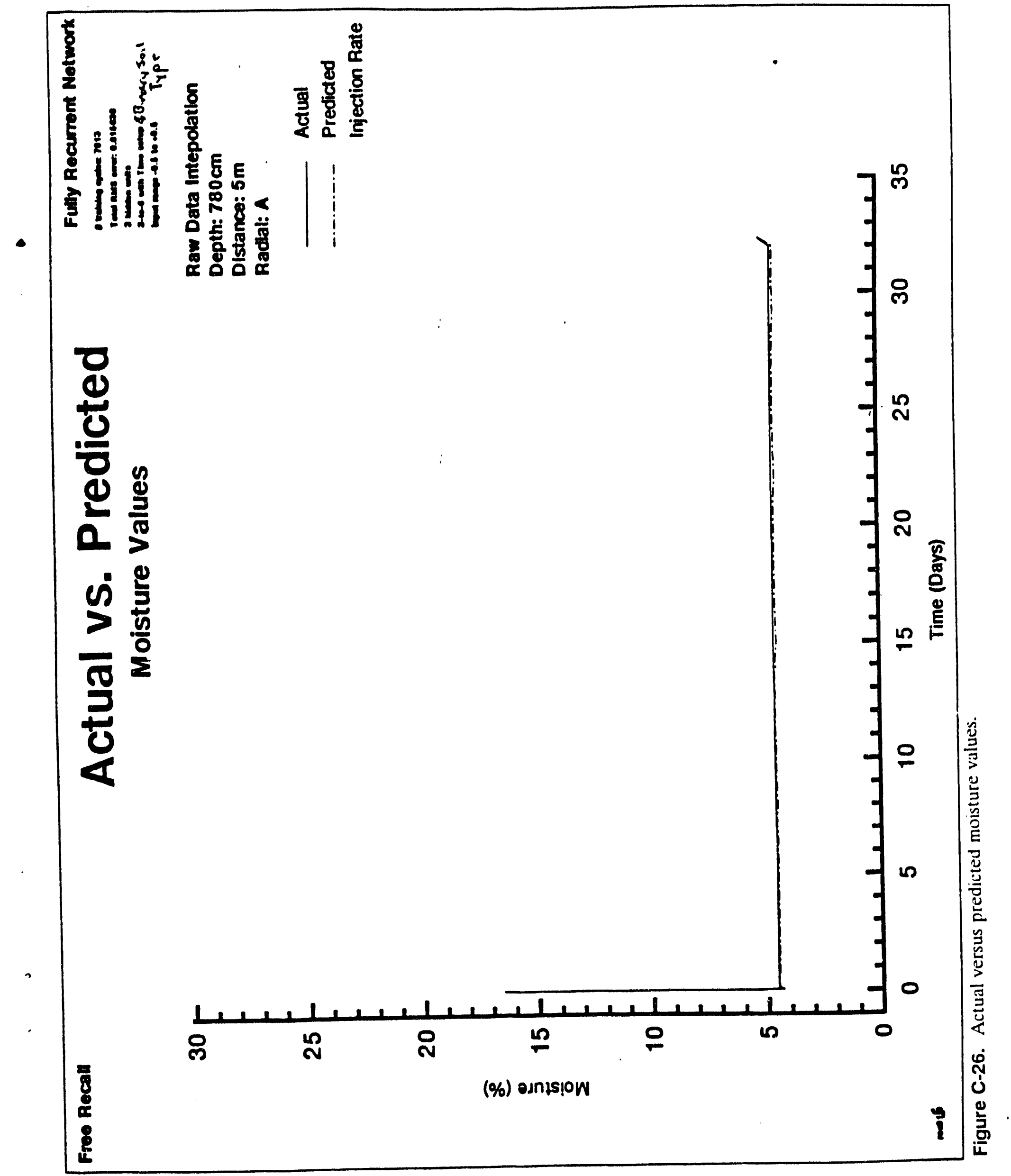




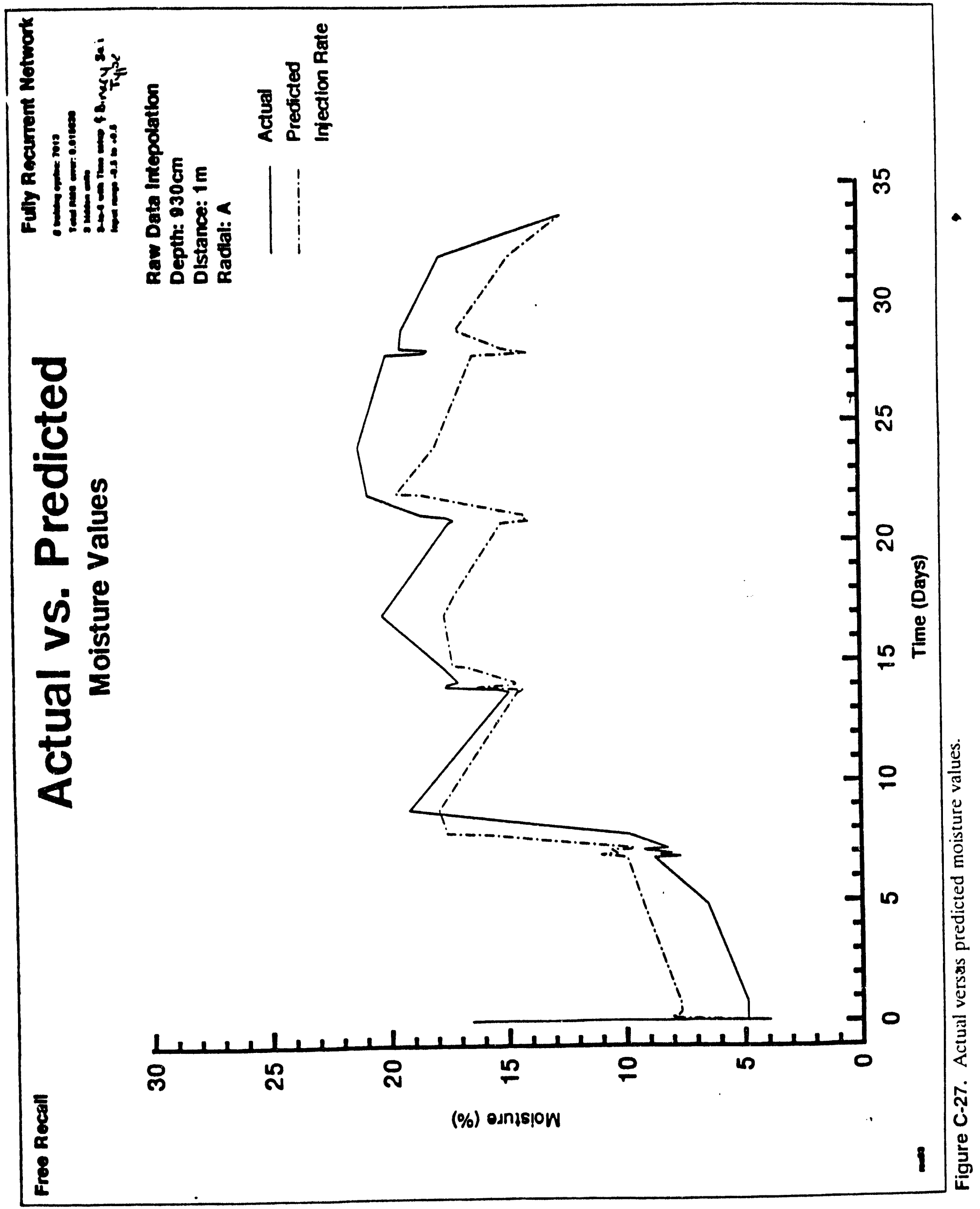

C- 32 


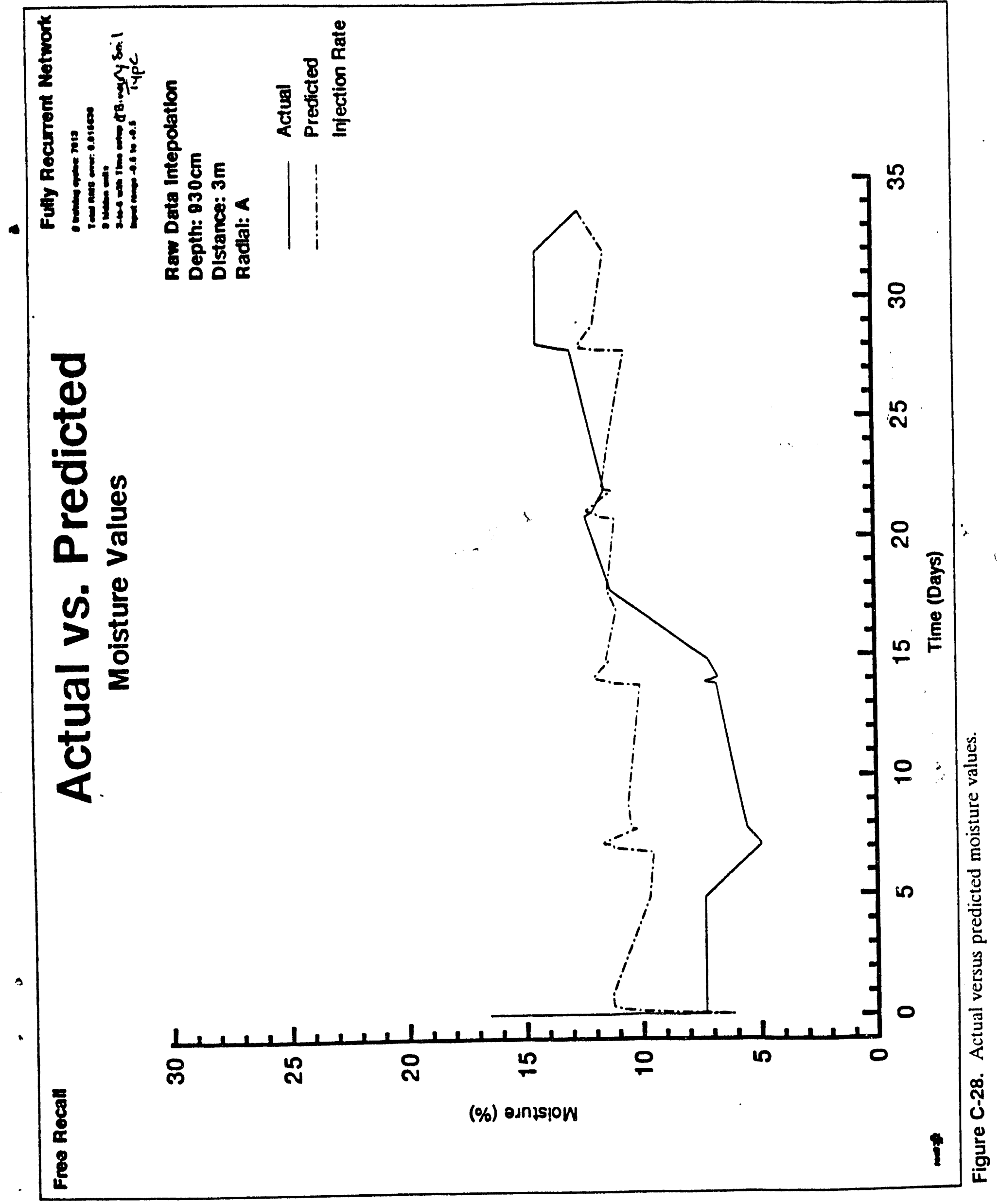




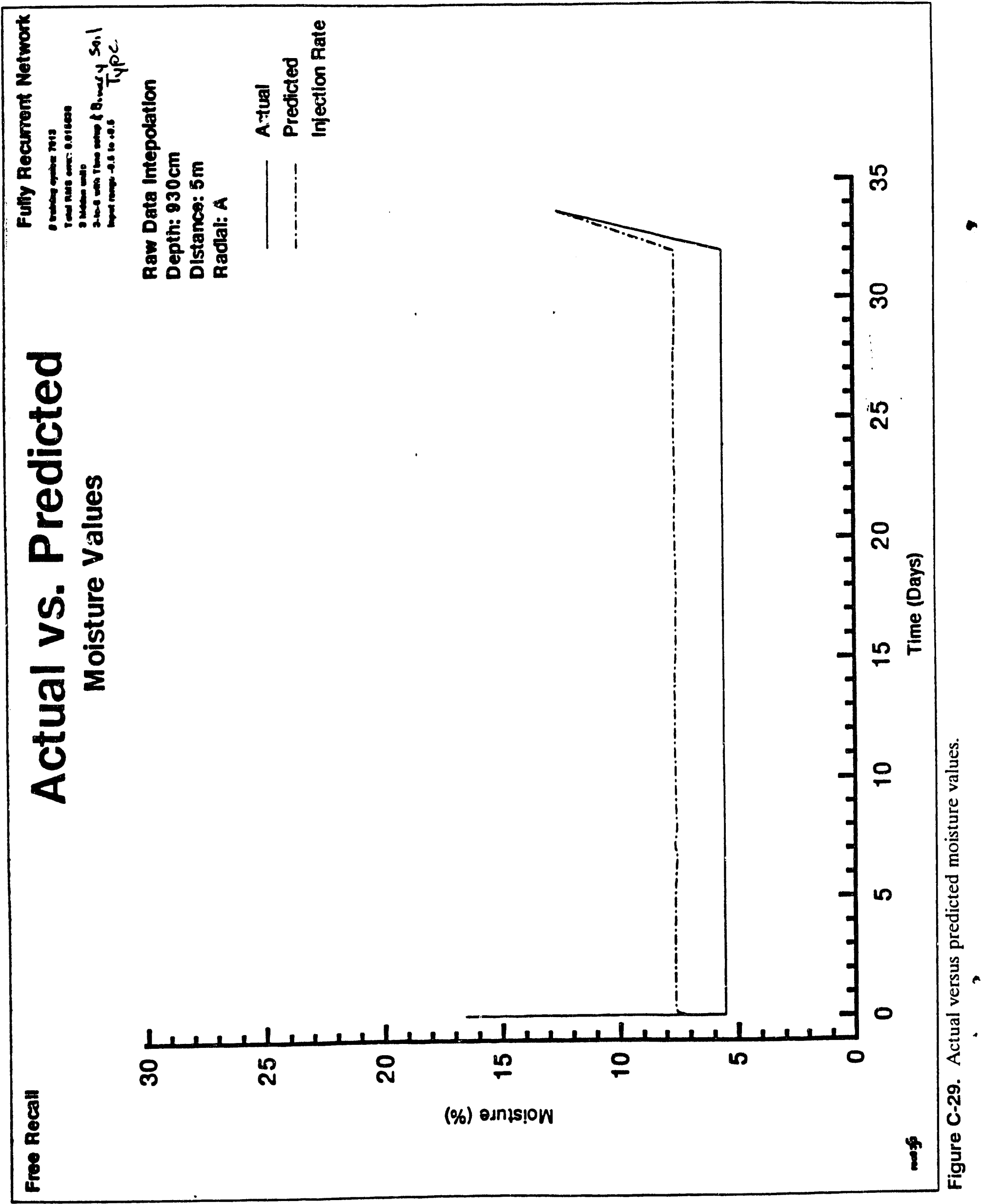

C-34 


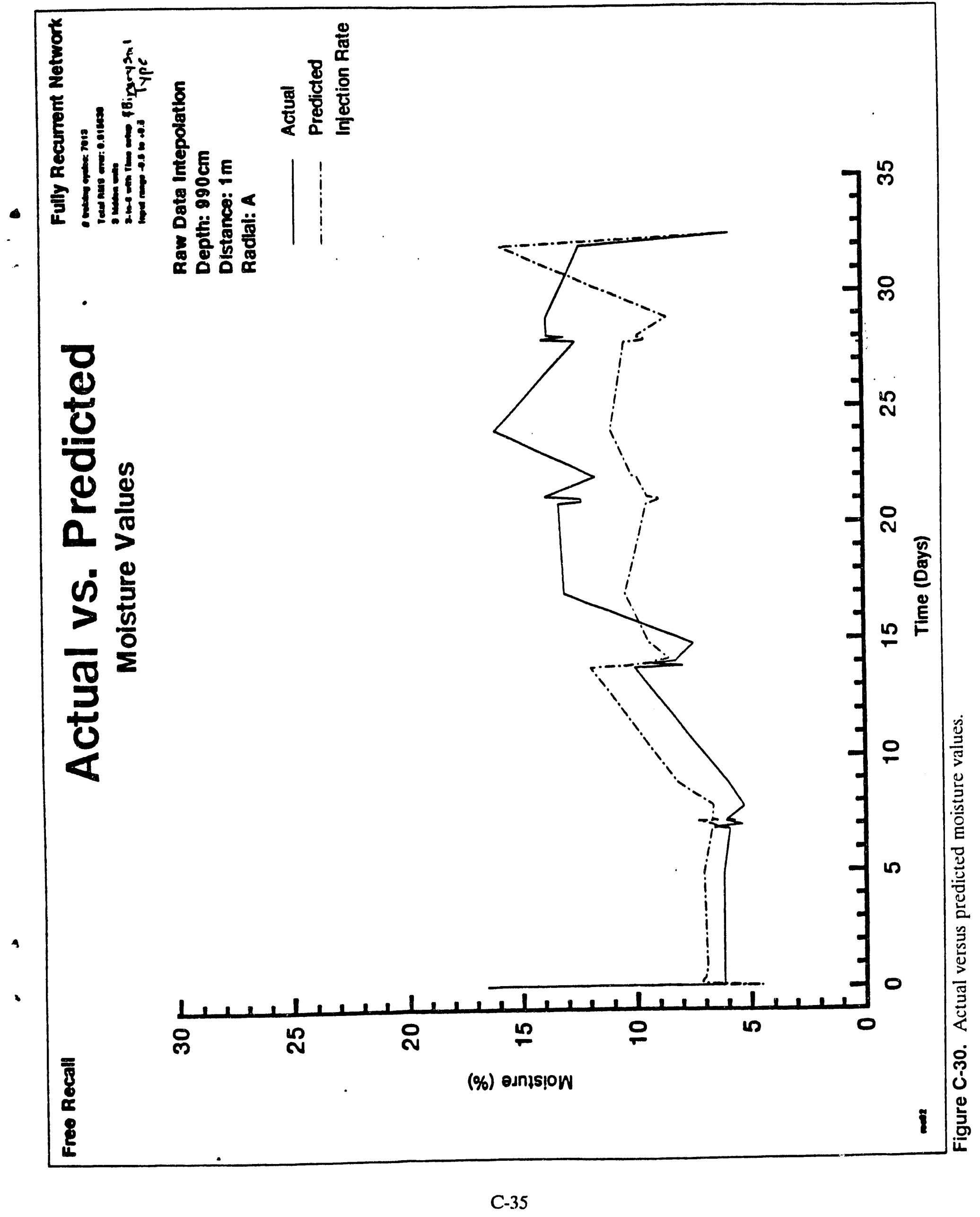




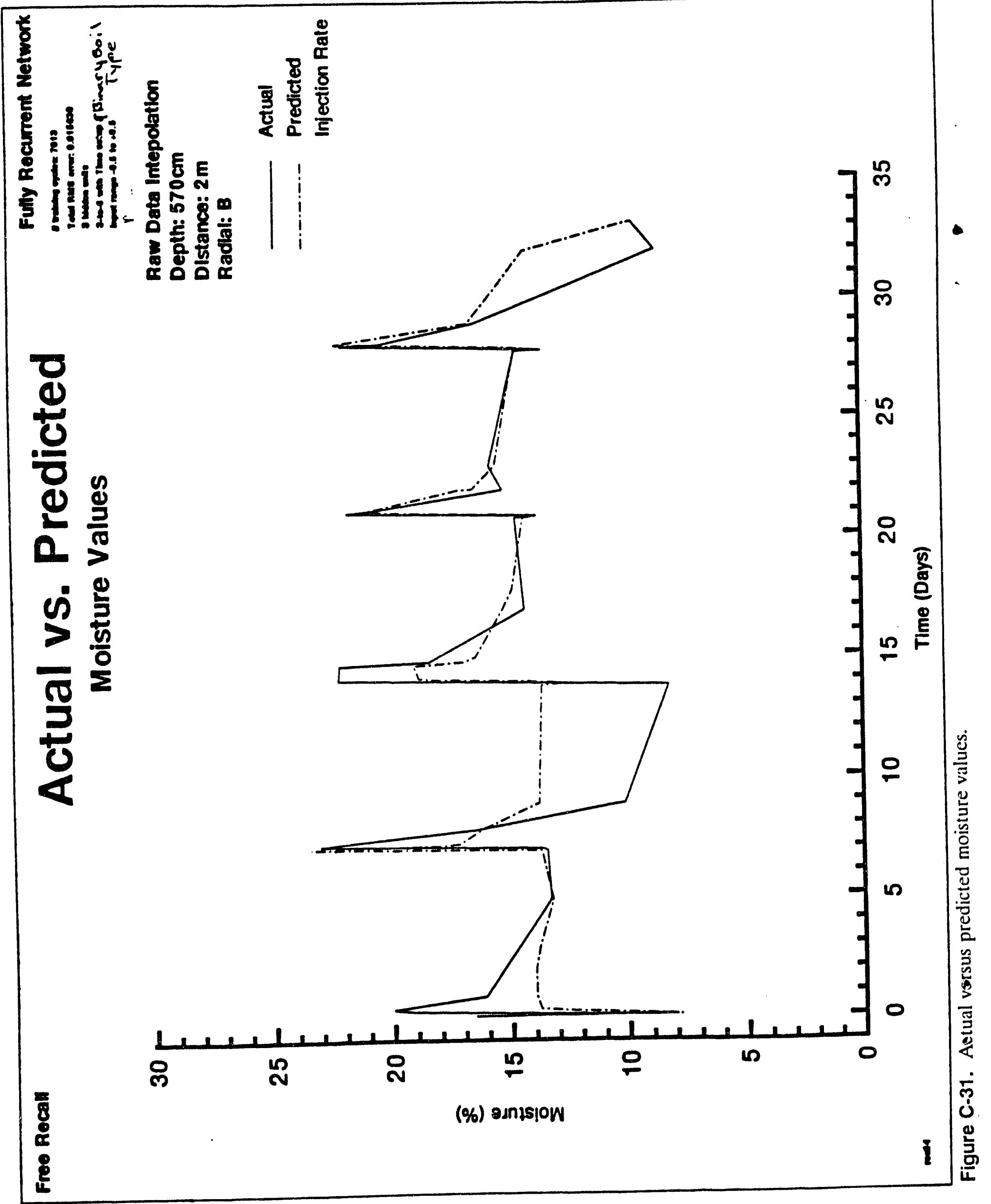



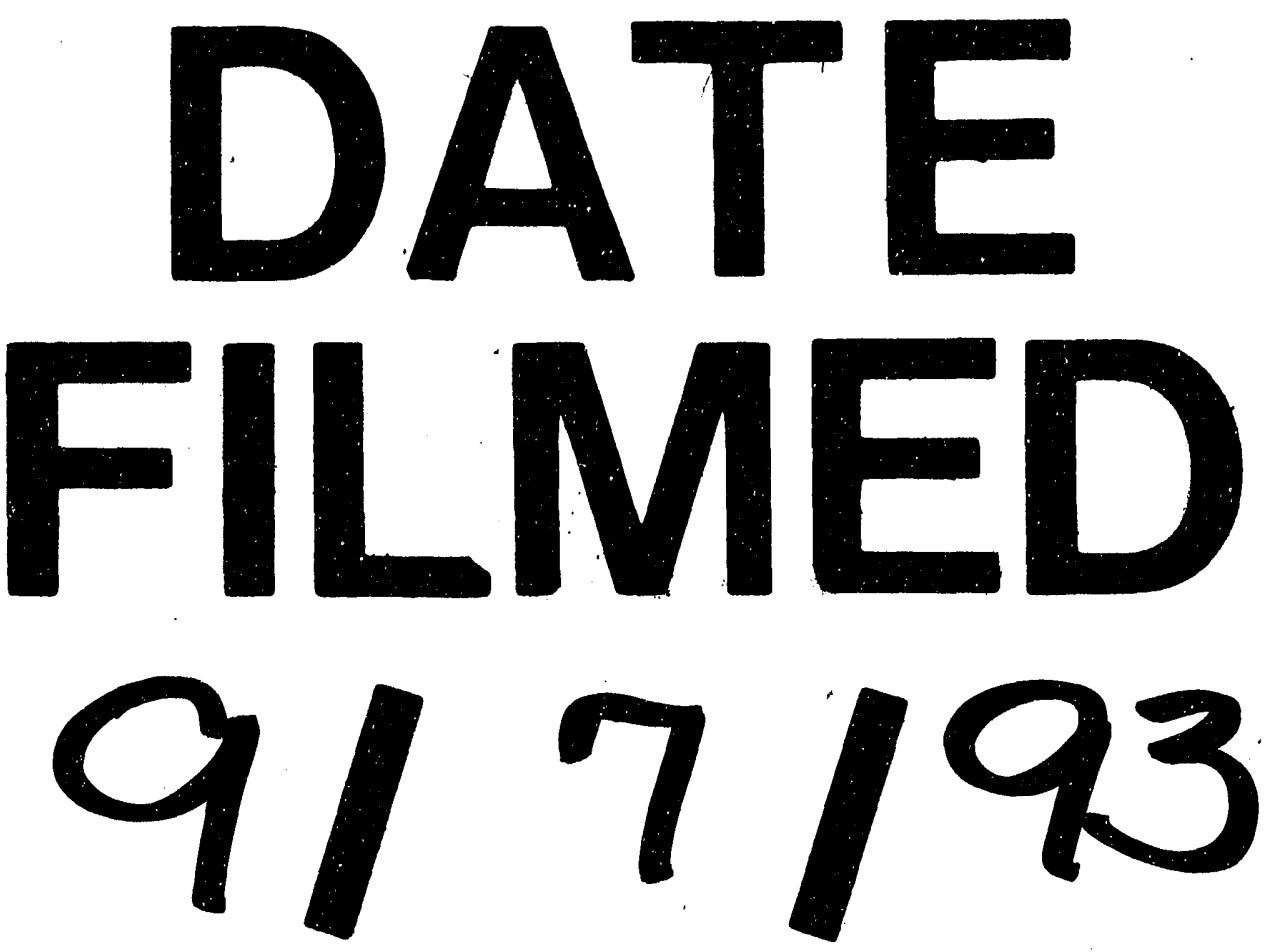
